\title{
Emergence of Rigid Polycrystals from Atomistic Systems with Heitmann-Radin Sticky Disk Energy
}

\author{
Manuel Friedrich, Leonard Kreutzid \& Bernd Schmidt
}

\author{
Communicated by A. GARRONI
}

\begin{abstract}
We investigate the emergence of rigid polycrystalline structures from atomistic particle systems. The atomic interaction is governed by a suitably normalized pair interaction energy, where the 'sticky disk' interaction potential models the atoms as hard spheres that interact when they are tangential. The discrete energy is frame invariant and no underlying reference lattice on the atomistic configurations is assumed. By means of $\Gamma$-convergence, we characterize the asymptotic behavior of configurations with finite surface energy scaling in the infinite particle limit. The effective continuum theory is described in terms of a piecewise constant field delineating the local orientation and micro-translation of the configuration. The limiting energy is local and concentrated on the grain boundaries, that is, on the boundaries of the zones where the underlying microscopic configuration has constant parameters. The corresponding surface energy density depends on the relative orientation of the two grains, their microscopic translation misfit, and the normal to the interface. We further provide a fine analysis of the surface energies at grain boundaries both for vacuum-solid and solid-solid phase transitions. The latter relies fundamentally on a structure result for grain boundaries showing that, due to the extremely brittle setup, interpolating boundary layers near cracks are energetically not favorable.
\end{abstract}

\section{Introduction}

Most inorganic solids in nature are polycrystals. They are composed of microscopic crystallites (grains) of varying size and orientation in which the atoms are arranged in a periodic, crystalline pattern. In spite of their ubiquity, it remains poorly understood why in these materials such highly regular structures develop at the microscale. The core challenge is to investigate the phenomenon of crystallization, that is, the tendency of atoms to self-assemble into a crystal structure. An ultimate solution would be to understand this as a consequence of the interatomic 
interactions, where such interactions are determined by the laws of quantum mechanics.

In view of the current state of research, however, the crystallization question seems out of reach in this generality. It is thus necessary to consider reduced models and to study simplified theories which, however, retain essential features of the interatomic interactions. We follow this route by restricting to zero temperature and by describing our system in the frame of Molecular Mechanics [1,30,37] as a classical system of particles, whose interaction is given in terms of an empirical pair interaction potential. Moreover, we consider planar rather than three-dimensional models. Given a configuration $X=\left\{x_{1}, \ldots, x_{N}\right\} \subset \mathbb{R}^{2}$ consisting of a finite number of particles, their configurational energy $\mathcal{E}(X)$ takes the form

$$
\mathcal{E}(X)=\frac{1}{2} \sum_{i \neq j} V_{\text {pair }}\left(\left|x_{i}-x_{j}\right|\right),
$$

where $V_{\text {pair }}:[0,+\infty) \rightarrow \overline{\mathbb{R}}$ denotes the pair potential. (The factor $1 / 2$ accounts for double counting.) Such potentials typically are repulsive for close-by atoms while two atoms at larger distances (yet still in their interaction range) exert attractive forces on each other. The latter favors the formation of clusters, whereas the shortrange repulsion guarantees that the atoms keep a minimal distance.

Notably, even for commonly used models such as the Lennard-Jones potential, the crystallization problem is still open beyond the one-dimensional setting. (In one dimension, the situation is considerably easier: crystallization at zero temperature for Lennard-Jones interactions is shown in [31]. Recent results for positive temperature including an analysis of boundary layers are obtained in [34,35]. For results on dimers we refer to [6,29].) The first rigorous results for a two-dimensional system were achieved in $[32,33,43]$; see also the recent paper [18]. For the very special choice of the 'Heitmann-Radin sticky disk' interaction potential

$$
V_{\text {sticky }}(r)= \begin{cases}+\infty & \text { if } r<1 \\ -1 & \text { if } r=1 \\ 0 & \text { if } r>1\end{cases}
$$

it was shown in [33] that ground states, that is, minimizers under the cardinality constraint $\# X=N$, crystallize: they are subsets of the triangular lattice. The potential $V_{\text {sticky }}$ is pictured schematically in Fig. 1.

On the one hand, it draws its motivation from being the most basic choice of a potential featuring the properties discussed above. On the other hand, it models extremely brittle materials and might be viewed as an 'infinitely brittle' limiting model for more generic interaction potentials, in which the hard core radius, the equlibrium distance, and the interaction range coincide. Slightly more general potentials are discussed in [43] which, however, do not allow for soft elastic interactions either. Still only partial results are available for more general potentials or higher dimensions, see [7] for a recent survey. Most noteworthy, [21,47] in two and [24] in three dimensions prove that crystalline structures have optimal bulk energy scaling and crystals are ground states subject to their own boundary conditions. 


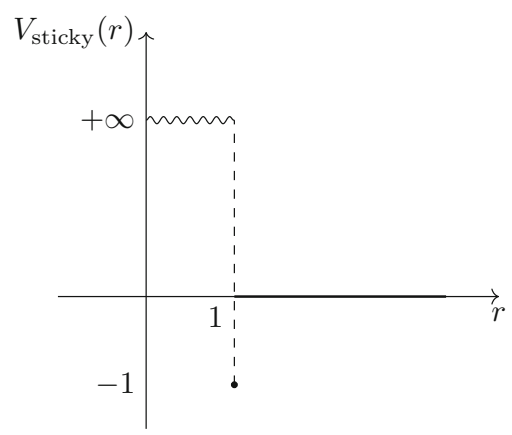

Fig. 1. The interaction potential $V_{\text {sticky }}$

Such conditions, however, are insufficient, respectively, prohibitive in view of our goal to investigate the emergence of polycrystals. For this task, it is indispensable to both work at the surface energy scale, which is much finer than the bulk scaling, and to allow for free boundary conditions.

The ground states of sticky disk potentials in two dimensions are by now very well understood, and not only on the atomic microscale. In [3] the macroscopic shape was identified as being the Wulff shape of an associated crystalline perimeter functional. Fine properties and surface fluctuations were investigated in [45] and quantified in terms of an $N^{3 / 4}$ law (see the comment below (1.2)). Sharp constants for this law were then established in [17] and the uniqueness of ground states was characterized in [19]. We also mention extensions to other crystals [16,40,42] and dimers $[26,27]$. By way of contrast, in dimension three or higher the recent results $[11,39,41]$ characterize optimal energy configurations within classes of lattices and are in this sense conditional to crystallization.

The main objective of our contribution is to advance our understanding of (microscopic) crystallization and formation of macroscopic clusters beyond ground states and single crystals. Indeed, all of the aforementioned results ultimately rely on the emergence of a single crystal which is supported on a unique periodic structure. Restricting our analysis to the basic Heitmann-Radin sticky disk potential (1.1), we succeed in deriving a rather complete picture on the formation of general polycrystals by considering the $\Gamma$-limit for the interaction energy in the surface energy regime in the infinite particle limit. (We refer to $[8,14]$ for an exhaustive treatment of $\Gamma$-convergence.) The first relevant steps in this direction were obtained in [20], where the authors prove a compactness result for polycrystals and identify the $\Gamma$-limit in the case of a single crystal limiting configuration. In the present work, we prove a full $\Gamma$-convergence result and provide a limiting continuum model consisting of grains that are characterized by a rotation and, in addition, a microtranslation. We also analyze in depth the surface energy of grain boundaries both for vacuum-solid and solid-solid phase transitions.

We proceed to describe our particle model in more detail. The minimal energy of a configuration $X_{N}=\left\{x_{1}, \ldots, x_{N}\right\} \subset \mathbb{R}^{2}$ of $N$ particles has been determined 
already in [32]:

$$
\min \left\{\mathcal{E}\left(X_{N}\right): \# X_{N}=N\right\}=-\lfloor 3 N-\sqrt{12 N-3}\rfloor \approx-3 N+\mathrm{O}(\sqrt{N}) .
$$

The leading order term $-3 N$ comes from $N-\mathrm{O}(\sqrt{N})$ atoms in the bulk, each having six neighbors. The lower order term $\sim \sqrt{N}$ is due to missing neighbors of a number $\mathrm{O}(\sqrt{N})$ of atoms at the boundary and is thus a surface energy. (The aforementioned $N^{3 / 4}$ law quantifies the surprisingly large possible deviations of ground states from the macroscopic Wulff shape which involve a number of $\sim N^{3 / 4} \gg \sqrt{N}$ particles.)

As polycrystals will not be ground states in general, but rather metastable states with surface energy contributions from atoms at individual grain boundaries, we proceed to address the class of all configurations at the finite surface energy scaling, that is, we consider $X_{N} \subset \mathbb{R}^{2}, \# X_{N}=N$, with bounded normalized energy

$$
\frac{\mathcal{E}\left(X_{N}\right)+3 N}{\sqrt{N}}=\frac{1}{2 \sqrt{N}} \sum_{x \in X_{N}}\left(6+\sum_{y \in X_{N} \backslash\{x\}} V_{\text {sticky }}(|x-y|)\right)
$$

as $N \rightarrow \infty$. Here, we have subtracted the minimal energy -3 per particle times the number of particles and rescaled with $\sqrt{N}$.

The diameter of an $N$-particle configuration $X_{N}$ with energy given in (1.2) is $\sim \sqrt{N}$. To obtain configurations which are contained in a bounded domain, we therefore rescale the configuration by a factor $\varepsilon:=1 / \sqrt{N}$, that is, $X_{\varepsilon}:=\varepsilon X_{N}$. We then study the asymptotics of the energy $E_{\varepsilon}\left(X_{\varepsilon}\right)$ where the energy functional $E_{\varepsilon}$ is defined on finite point sets $X \subset \mathbb{R}^{2}$ by

$$
E_{\varepsilon}(X)=\frac{1}{2} \sum_{x \in X} \varepsilon\left(6+\sum_{y \in X \backslash\{x\}} V_{\text {sticky }}\left(\frac{|x-y|}{\varepsilon}\right)\right) .
$$

This will allow us to pass to a macroscopic description as $\varepsilon \rightarrow 0$. In what follows, we consider the energy $E_{\varepsilon}$ in (1.3) without cardinality constraint since the energy has already been normalized with respect to the minimal energy per particle.

Our main results are a full $\Gamma$-convergence proof for the functionals $E_{\varepsilon}$ towards a surface energy functional (Theorem 2.3) and a detailed analysis of the limiting continuum surface energy density (Proposition 2.2 and Theorem 2.5). We also prove a corresponding compactness result for bounded energy sequences (Theorem 2.1), which turns out to be comparatively straightforward. The proofs in fact also provide a rather complete picture of the structure of grain boundaries. We collect these findings of independent interest in Theorem 5.4. Our continuum description keeps track not only of the orientation angles of various grains but depends additionally on a micro-translation vector which in particular measures the translational offset of two lattices with the same orientation. Indeed, the introduction of such an augmented field does not only provide a finer characterization of the continuum limit, but turns out to be crucial when polycrystals with multiple solid-solid grain boundaries are considered.

The limiting surface energy $\varphi$ is a function of the relative orientation of the two grains, their microscopic translation misfit, and the normal to the interface. For solid-vacuum surfaces this was identified in $[3,20]$ as the Finsler norm whose unit 
ball is shaped like a Voronoi cell of the lattice in the solid part. In other words, this is just the surface energy density of the crystal perimeter. For solid-solid interfaces, however, the problem is considerably more subtle as there are atomic interactions across the interface. In softer materials, one expects dislocations to accumulate and elastic strain to concentrate near such grain boundaries. We refer to [23,38] for recent mathematical developments on substantiating the Read-Shockley formula, see [44]; in such a regime. By way of contrast, within our extremely brittle setup, generically $\varphi$ turns out to be given by the sum of the solid-vacuum surface energies of the two grains. Here, the term generic refers to the fact that the surface energy may be smaller only for a countable number of mismatch angles between the two lattices, and corresponding micro-translations contained in a finite number of spheres.

We proceed with some comments on the general proof strategy. As is customary for variational limits with interfacial energies, the density $\varphi$ is expressed in terms of a cell formula minimizing the asymptotic surface energy between two grains separated by a flat grain boundary. In such cell problems, it is instrumental to pass from a mere $L^{1}$-convergence to fixed boundary values in order to match the $\Gamma$ lim inf and $\Gamma$-lim sup inequalities. Motivated by $[5,25,46]$ for vectorial problems in liquid-liquid phase transitions and $[13,15,36]$ in solid-solid phase transitions, we use a cut-off construction, the so-called fundamental estimate, to replace an asymptotic realization by the exact attainnment of converging boundary values in a first step. Here, our extremely brittle set-up on the one hand renders geometric rigidity estimates easier as compared to, for example, $[13,15]$. On the other hand, this calls for carefully refined cut-off constructions since very small modifications in the configurations may induce a lot of energy. However, in contrast to $[13,15]$, a cell problem with converging boundary data turns out to be insufficient in the presence of multiple grain boundaries. Thus, a further step is needed to show that they can be replaced by fixed boundary values. Also this passage is subtle due to our rigid set-up which requires a thorough analysis of possible touching points of two lattices (points with distance $\varepsilon$ ). Finally, let us also mention that related, very general $\Gamma$-convergence results for elastic materials exhibiting discontinuities along surfaces, see for example $[4,10,28]$, do not apply to our situation. Most notably, in [28], a model similar to ours featuring rigid grains is considered. Unfortunately, these results cannot be used in our setting as they fundamentally rely on continuous surface interactions.

At the core of our proofs, there are two key steps to which we devote Sections 5 and 6, respectively. Firstly, Lemma 5.1 allows us to reduce the cell formula to two lattices only. An expanded version of this observation is detailed in Theorem 5.4. It shows that in our brittle set-up there are no interpolating boundary layers at interfaces. This is done by employing techniques from graph theory in order to exclude inclusions of grains of different orientation as the prescribed boundary datum. The basic idea behind its proof is that to each admissible configuration one can associate its bond-graph and for this graph such inclusions induce nontriangular faces which in turn lead to fewer bonds than a competitor without such inclusions. This can be quantified via the face defect, see definition (5.4). Once established, this in particular results in a largely simplified analysis of the interaction 
energy with vacuum as compared to [20], see Lemma 6.1. More importantly, it is crucial for the second main ingredient of the proof: the quantification of solid-solid interactions with the help of Lemma 6.2, which clarifies when the surface energy can be smaller than twice the interaction energy with vacuum and plays a pivotal role in order to show that converging boundary values can be replaced by fixed ones. This can be understood as a rigidity theorem for the mismatch-angle between two grains: the generically expected interaction energy can exceed the grain boundary energy only for finitely many mismatch angles depending on the excess. Its proof relies on the fact that such an energy gap can only occur if the two lattices have many touching points (points with distance $\varepsilon$ ). This entails that the touching points of the two lattices have to be rather equi-distributed along the interface. This, however, can only happen in a periodic landscape, which reduces the possible mismatch-angle to a finite set. Many further ingredients of our proofs are more standard (blow-up, density arguments, fundamental estimate, ...), but technically challenging in our case since the energy is very rigid and thus very sensitive to small changes of the configuration.

The paper is organized as follows: in Section 2 we introduce the model and present the main results. Section 3 is devoted to the proofs of compactness and $\Gamma$-convergence. They fundamentally rely on a fine characterization of the surface energy density whose proof is postponed to Sections 4-7. In Section 4 we address the fundamental estimate and in Section 7 we show that converging boundary values can be replaced by fixed ones. Sections 5 and 6 are devoted to the reduction of the cell formula to two lattices only and to the characterization of solid-vacuum/solidsolid interactions at grain boundaries, respectively.

\section{Setting of the Problem and Main Results}

In this section we introduce our model, give basic definitions, and present our main results.

\subsection{Configurations and Atomistic Energy}

In what follows we always assume that $X$ is a finite subset of $\mathbb{R}^{2}$. We denote by $V_{\text {sticky }}:[0,+\infty) \rightarrow \overline{\mathbb{R}}$ the Heitmann-Radin potential defined in (1.1), see Fig. 1. By $\varepsilon>0$ we denote the atomic spacing. The normalized atomistic energy $E_{\varepsilon}$ of a given configuration $X$ is given by (1.3). The notion normalized has been explained in the introduction and is chosen in such a way that an infinite triangular lattice with spacing $\varepsilon$ has energy zero. Equivalently, the energy can be expressed in terms of the neighborhoods of the atoms. To this end, we introduce the neighborhood of $x \in X$ by

$$
\mathcal{N}_{\varepsilon}(x)=\{y \in X:|x-y|=\varepsilon\}
$$

If $\varepsilon=1$, we omit the subscript $\varepsilon$ and just write $\mathcal{N}(x)$ for simplicity. In view of $V_{\text {sticky }}(r)=\infty$ for $r \in(0,1)$, an elementary geometric argument shows that for 
configurations $X$ with $E_{\varepsilon}(X)<+\infty$ it holds that

$$
\# \mathcal{N}_{\varepsilon}(x) \leqq 6 \text { for all } x \in X
$$

In particular, if $\# \mathcal{N}_{\varepsilon}(x)=6$, the neighbors form a regular hexagon with center $x$ and diameter $2 \varepsilon$. By (1.1) and (1.3) we can now rewrite the energy as

$$
E_{\varepsilon}(X)=\frac{1}{2} \sum_{x \in X} \varepsilon\left(6-\# \mathcal{N}_{\varepsilon}(x)\right) .
$$

Additionally, for $X \subset \mathbb{R}^{2}$ and Borel sets $B \subset \mathbb{R}^{2}$, we define a localized version of the energy by

$$
E_{\varepsilon}(X, B)=\frac{1}{2} \sum_{x \in X \cap B} \varepsilon\left(6-\# \mathcal{N}_{\varepsilon}(x)\right)
$$

\subsection{Basic Definitions}

This subsection is devoted to basic notions which we will use throughout the paper.

Notation We let $\mathbb{S}^{1}=\left\{x \in \mathbb{R}^{2}:|x|=1\right\}$. Given $v \in \mathbb{S}^{1}$, we denote by $\nu^{\perp} \in \mathbb{S}^{1}$ the unit vector obtained by rotating $v$ by $\pi / 2$ in a clockwise sense. The scalar product between two vectors $x, y \in \mathbb{R}^{2}$ is denoted by $\langle x, y\rangle$. Without further notice, we sometimes identify vectors $x \in \mathbb{R}^{2}$ with elements of $\mathbb{C}$. In particular, we identify rotations in the plane with a multiplication with a unit vector in $\mathbb{C}$ : namely, the rotation of $x \in \mathbb{R}^{2}$ by an angle $\theta \in[0,2 \pi)$ is indicated by $e^{i \theta} x$. For $t \in \mathbb{R}$, we write $\lfloor t\rfloor=\max \{k \in \mathbb{Z}: k \leqq t\}$ and $\lceil t\rceil=\min \{k \in \mathbb{Z}: k \geqq t\}$.

We denote by $\mathcal{L}^{2}$ and $\mathcal{H}^{1}$ the two-dimensional Lebesgue measure and the onedimensional Hausdorff measure, respectively. We write $\chi_{E}$ for the characteristic function of any $E \subset \mathbb{R}^{2}$, which is 1 on $E$ and 0 otherwise. If $E$ is a set of finite perimeter, we denote its essential boundary by $\partial^{*} E$, see [2, Definition 3.60]. For $r>0$ and $x \in \mathbb{R}^{2}$, we denote by $B_{r}(x)$ the open ball of radius $r$ centered in $x$. For simplicity, we write $B_{r}$ if $x=0$. Given $A \subset \mathbb{R}^{2}, \tau \in \mathbb{R}^{2}$, and $\lambda \in \mathbb{R}$, we define

$$
\begin{aligned}
& A+\tau=\{x+\tau: x \in A\}, \quad \lambda A=\{\lambda x: x \in A\} \text { and } \\
& (A)_{\varepsilon}=\left\{x+y: x \in A, y \in B_{\varepsilon}\right\} .
\end{aligned}
$$

For $x_{1}, x_{2} \in \mathbb{R}^{2}$, we define the line segment between $x_{1}$ and $x_{2}$ by

$$
\left[x_{1} ; x_{2}\right]=\left\{\lambda x_{1}+(1-\lambda) x_{2}: \lambda \in[0,1]\right\} .
$$

By $Q^{v}=\left\{y \in \mathbb{R}^{2}:-\frac{1}{2} \leqq\langle y, v\rangle<\frac{1}{2},-\frac{1}{2} \leqq\left\langle y, v^{\perp}\right\rangle<\frac{1}{2}\right\}$ we denote the halfopen unit cube in $\mathbb{R}^{2}$ with center zero and two sides parallel to $v \in \mathbb{S}^{1}$. Moreover, we define the half-cubes

$$
Q^{\nu, \pm}=\left\{y \in Q^{\nu}: \pm\langle\nu, y\rangle \geqq 0\right\} .
$$

Here and in what follows, we will frequently use the notation \pm to indicate that a property holds for both signs + and - . In a similar fashion, for $x \in \mathbb{R}^{2}$ and $\rho>0$ 
we define $Q_{\rho}^{\nu}(x):=x+\rho Q^{\nu}$ and $Q_{\rho}^{\nu, \pm}(x):=x+\rho Q^{\nu, \pm}$. For $\rho=1$, we write $Q^{v}(x)$ instead of $Q_{1}^{v}(x)$ for simplicity. For $\varepsilon>0$ and $Q_{\rho}^{v}(x)$ we introduce the notation of boundary regions

$$
\partial_{\varepsilon}^{ \pm} Q_{\rho}^{\nu}(x)=x+\left\{y \in \overline{Q_{\rho+10 \varepsilon}^{v} \backslash Q_{\rho-10 \varepsilon}^{\nu}}: \pm\langle\nu, y\rangle \geqq 5 \varepsilon\right\}
$$

see also Fig. 3 for an illustration. For $\rho=1$, we write $\partial_{\varepsilon}^{ \pm} Q^{v}(x)$ instead of $\partial_{\varepsilon}^{ \pm} Q_{\rho}^{v}(x)$. The triangular lattice We define the triangular lattice as the set of points given by

$$
\mathscr{L}:=\{p+q \omega: p, q \in \mathbb{Z}\}
$$

where $\omega:=\frac{1}{2}+\frac{i}{2} \sqrt{3} \in \mathbb{C}$.

The set of lattice isometries We denote by $\mathbb{A}$ the set of rotations by angles in $\left[0, \frac{\pi}{3}\right)$ equipped with the metric of the one-dimensional torus, that is, $\mathbb{A}=\mathbb{R} / \frac{\pi}{3} \mathbb{Z}$. In a similar fashion, we introduce the set of translations $\mathbb{T}=\mathbb{R}^{2} / \mathscr{L}=\mathbb{C} / \mathscr{L}$. We observe that each translation $\tau \in \mathbb{T}$ can be represented by a vector in

$$
\left\{\lambda_{1}+\lambda_{2} \omega: 0 \leqq \lambda_{1}<1,0 \leqq \lambda_{2}<1\right\} .
$$

We introduce the set of lattice isometries by

$$
\mathcal{Z}:=(\mathbb{A} \times \mathbb{T} \times\{1\}) \cup\{\mathbf{0}\}
$$

where for each $\theta \in \mathbb{A}$ and $\tau \in \mathbb{T}$ the triple $z=(\theta, \tau, 1) \in \mathcal{Z}$ represents the rotated and translated lattice

$$
\mathscr{L}(z)=\mathscr{L}(\theta, \tau, 1):=e^{i \theta}(\mathscr{L}+\tau) .
$$

Here, the entry 1 encodes that a lattice is present. On the contrary, $\mathbf{0}=(0,0,0) \in$ $\mathbb{A} \times \mathbb{T} \times\{0\}$ represents the empty set, also referred to as vacuum in what follows. We set

$$
\mathscr{L}(\mathbf{0})=\emptyset .
$$

Note that $\mathbb{A} \simeq \mathbb{S}^{1}$ and $\mathbb{T} \simeq \mathbb{S}^{1} \times \mathbb{S}^{1}$. Therefore, the three-dimensional set $\mathcal{Z}$ can naturally be embedded into $\mathbb{R}^{7}$. We endow $\mathcal{Z}$ with the product topology, that is, $z_{j}=\left(\theta_{j}, \tau_{j}, 1\right) \rightarrow z=(\theta, \tau, 1)$ if and only if $\theta_{j} \rightarrow \theta$ in $\mathbb{A}$ and $\tau_{j} \rightarrow \tau$ in $\mathbb{T}$. Moreover, $z_{j} \rightarrow \mathbf{0}$ if and only if $z_{j}=\mathbf{0}$ for all $j$ large enough. For a set $A \subset \mathbb{R}^{2}$, $z \in \mathcal{Z}$, and a configuration $X$ with $E_{\varepsilon}(X)<+\infty$, we say that $X$ coincides with the lattice $\varepsilon \mathscr{L}(z)$ on $A$, written $X=\varepsilon \mathscr{L}(z)$ on $A$, if

$$
X \cap A=(\varepsilon \mathscr{L}(z)) \cap A .
$$

The state space For $A \subset \mathbb{R}^{2}$, we introduce the space of piecewise constant functions $P C(A ; \mathcal{Z})$ with values in $\mathcal{Z}$ as functions of the form

$$
u=\sum_{j=1}^{\infty} \chi_{G_{j}} z_{j},
$$


where $\left\{z_{j}\right\}_{j} \subset \mathcal{Z} \backslash\{\boldsymbol{0}\}$ are pairwise distinct and $G_{j} \subset A$ are pairwise disjoint sets satisfying $\mathcal{L}^{2}\left(\bigcup_{j=1}^{\infty} G_{j}\right)<\infty$ and

$$
\sum_{j=1}^{\infty} \mathcal{H}^{1}\left(\partial^{*} G_{j}\right)<+\infty
$$

Here, $\left\{G_{j}\right\}_{j}$ represent the grains of the polycrystal and $\left\{z_{j}\right\}_{j}$ the corresponding orientation and translation of the lattice. We remark that this space can be identified with

$$
P C(A ; \mathcal{Z})=\left\{u \in S B V(A ; \mathcal{Z}): \nabla u=0, \mathcal{L}^{2}(\{u \neq \mathbf{0}\})<+\infty, \mathcal{H}^{1}\left(J_{u}\right)<+\infty\right\} .
$$

Here, $u$ is a function in $S B V(A ; \mathcal{Z})$ in the sense that $u \in S B V\left(A ; \mathbb{R}^{7}\right)$ and $u$ takes values in $\mathcal{Z}$. The jump set of $u$ is denoted by $J_{u}$. The one-sided limits of $u$ at a jump point will be indicated by $u^{+}$and $u^{-}$in what follows, and the normal will be denoted by $v_{u}$. We refer to [2, Definition 4.21] for details on this space. In a similar fashion, we say $u \in P C_{\mathrm{loc}}\left(\mathbb{R}^{2} ; \mathcal{Z}\right)$ if $\left.u\right|_{A} \in P C(A ; \mathcal{Z})$ for all compact sets $A \subset \mathbb{R}^{2}$.

Identification of configurations with piecewise constant functions We now relate atomistic configurations $X$ to the state space defined above. Consider $x \in X \cap \mathscr{L}$ such that $\mathcal{N}(x) \subset \mathscr{L}$. Then, we define the open lattice Voronoi cell of $x$ by

$$
V(x)=x+\frac{1}{\sqrt{3}} e^{i \pi / 6} \operatorname{int}\left(\operatorname{conv}\left\{ \pm 1, \pm \omega, \pm \omega^{2}\right\}\right),
$$

where $\operatorname{conv}\{\cdot\}$ denotes the convex hull of a point set, and int the interior. In a similar fashion, if $x$ and the points in its neighborhood $\mathcal{N}_{\varepsilon}(x)$ lie in a scaled rotated and translated lattice $\varepsilon \mathscr{L}(z)$, for $\varepsilon>0$ and $z=(\theta, \tau, 1) \in \mathcal{Z}$, we define $V_{\varepsilon}^{z}(x)=x+$ $e^{i \theta} \varepsilon V(0)$. We also point out the implicit dependence on $\tau$ here, since $x=e^{i \theta}(v+\tau)$ for some $v \in \mathscr{L}$.

Given a configuration $X$ with $E_{\varepsilon}(X)<+\infty$, we now identify $X$ with a suitable function $u \in P C\left(\mathbb{R}^{2} ; \mathcal{Z}\right)$. Since $E(X)<+\infty$, we have $\# \mathcal{N}_{\varepsilon}(x) \leqq 6$ for all $x \in X$ with equality only if $\{x\} \cup \mathcal{N}_{\varepsilon}(x) \subset e^{i \theta(x)} \varepsilon(\mathscr{L}+\tau(x))$ for a unique pair $(\theta(x), \tau(x)) \in \mathbb{A} \times \mathbb{T}$. We set

$$
z(x)=(\theta(x), \tau(x), 1) \in \mathcal{Z} \quad \text { for all } x \in X \text { with } \# \mathcal{N}_{\varepsilon}(x)=6
$$

and define $u_{\varepsilon}^{X}: \mathbb{R}^{2} \rightarrow \mathcal{Z}$ by

$$
u_{\varepsilon}^{X}(x):= \begin{cases}z(x) \text { on } V_{\varepsilon}^{z(x)}(x) & \text { if } x \in X \text { with } \# \mathcal{N}_{\varepsilon}(x)=6 \\ \mathbf{0} & \text { else. }\end{cases}
$$

In what follows, if no confusion may arise, we write $u_{\varepsilon}$ instead of $u_{\varepsilon}^{X}$. We note that this definition is well posed in the sense that $V_{\varepsilon}^{z\left(x_{1}\right)}\left(x_{1}\right) \cap V_{\varepsilon}^{z\left(x_{2}\right)}\left(x_{2}\right)=\emptyset$ for all $x_{1}, x_{2} \in X, x_{1} \neq x_{2}$, with $\# \mathcal{N}_{\varepsilon}\left(x_{1}\right)=\# \mathcal{N}_{\varepsilon}\left(x_{2}\right)=6$. In fact, if this were not the case, one of the six atoms in $\mathcal{N}_{\varepsilon}\left(x_{1}\right)$ (forming a regular hexagon on $\partial B_{\varepsilon}\left(x_{1}\right)$ ) 


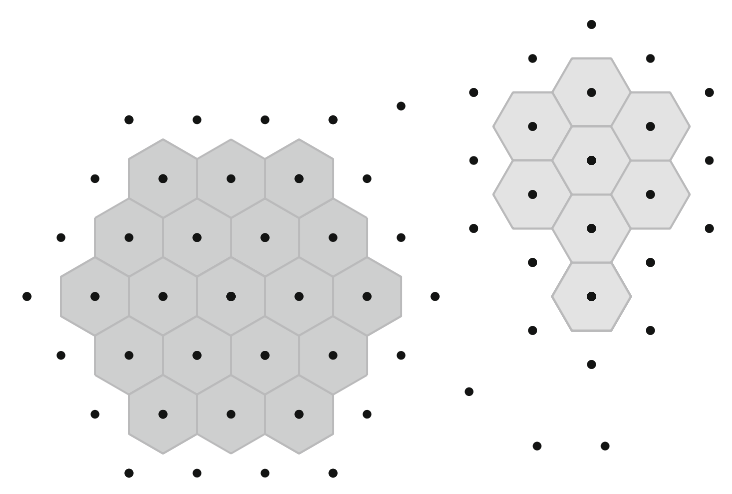

Fig. 2. A function $u_{\varepsilon}$ defined in (2.15): the different regions $\{u=z\}$ with $z \neq \mathbf{0}$ (here illustrated in different shades of gray) are made of unions of regular hexagons. The complement of those regions is the set $\{u=\mathbf{0}\}$

would have distance smaller than 1 to $x_{2}$. This contradicts $E_{\varepsilon}(X)<+\infty$. Clearly, $u_{\varepsilon}$ as defined in $(2.15)$ lies in $P C\left(\mathbb{R}^{2} ; \mathcal{Z}\right)$.

The function $u_{\varepsilon}$ for some finite energy configuration $X$ is illustrated in Fig. 2. We point out that the translation $\tau(x)$ induces a shift of the Voronoi cells by the vector $\varepsilon e^{i \theta(x)} \tau(x)$. This is the reason why we call the variable $\tau$ a micro-translation. Convergence Let $\left\{X_{\varepsilon}\right\}_{\varepsilon}$ be a sequence of configurations. We say that $X_{\varepsilon} \rightarrow u$ in $L_{\text {loc }}^{1}\left(\mathbb{R}^{2}\right)$ if $u_{\varepsilon} \rightarrow u$ in $L_{\text {loc }}^{1}\left(\mathbb{R}^{2} ; \mathcal{Z}\right)$, where $u_{\varepsilon}$ is given by $(2.15)$ for $X_{\varepsilon}$.

\subsection{Main Results}

We now formulate our main results. We start with a compactness result for sequences of configurations with bounded energy. Recall the definition for convergence of configurations in Sect. 2.2.

Theorem 2.1. (Compactness) Let $\left\{X_{\varepsilon}\right\}_{\varepsilon}$ be a sequence of configurations with

$$
\sup _{\varepsilon>0} E_{\varepsilon}\left(X_{\varepsilon}\right)<+\infty \text {. }
$$

Then, there exists a subsequence $\left\{\varepsilon_{k}\right\}_{k \in \mathbb{N}}$ with $\varepsilon_{k} \rightarrow 0$ and a function $u \in P C\left(\mathbb{R}^{2} ; \mathcal{Z}\right)$ such that $X_{\varepsilon_{k}} \rightarrow$ in $L_{\mathrm{loc}}^{1}\left(\mathbb{R}^{2}\right)$ as $k \rightarrow+\infty$.

For $\varepsilon>0$ and $\nu \in \mathbb{S}^{1}$, recall the definition of $\partial_{\varepsilon}^{ \pm} Q_{\rho}^{\nu}$ in (2.7). Recall also the coincidence with a lattice in (2.10). The following proposition introduces the density $\varphi: \mathcal{Z} \times \mathcal{Z} \times \mathbb{S}^{1} \rightarrow[0,+\infty)$ which appears in our continuum limiting functional (see Fig. 3 for an illustration):

Proposition 2.2. (Density) For every $z^{+}, z^{-} \in \mathcal{Z}, v \in \mathbb{S}^{1}, x_{0} \in \mathbb{R}^{2}$, and $\rho>0$ there exists

$$
\varphi\left(z^{+}, z^{-}, v\right)=\lim _{\varepsilon \rightarrow 0} \frac{1}{\rho} \min \left\{E_{\varepsilon}\left(X, Q_{\rho}^{v}\left(x_{0}\right)\right): X=\varepsilon \mathscr{L}\left(z^{ \pm}\right) \text {on } \partial_{\varepsilon}^{ \pm} Q_{\rho}^{v}\left(x_{0}\right)\right\},
$$

and is independent of $x_{0}$ and $\rho$. 


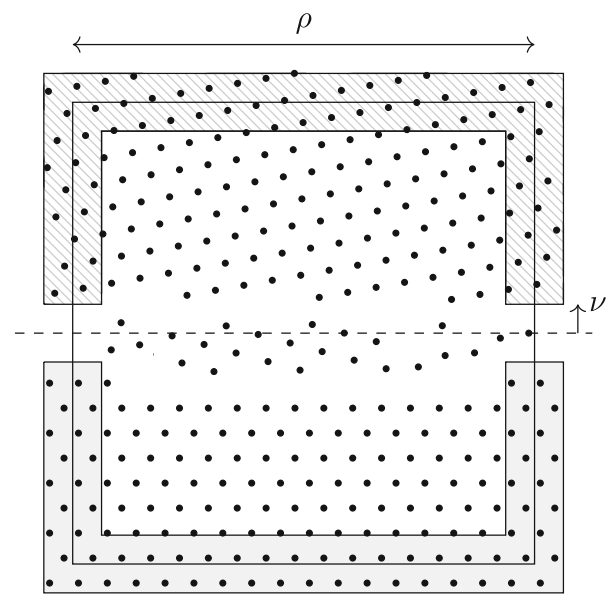

Fig. 3. Illustration of a competitor for the cell-problem on $Q_{\rho}^{v}$ in the definition of $\varphi$. On the light gray hatched and dark gray regions we have $X=\varepsilon \mathscr{L}\left(z^{ \pm}\right)$, respectively. We point out that the competitor is prescribed in a small neighborhood $\partial_{\varepsilon}^{-} Q_{\rho}^{v} \cup \partial_{\varepsilon}^{+} Q_{\rho}^{v}$ both inside and outside of the cube. (The thickness of the neighborhood is larger than the lattice spacing, see (2.7). Here, for illustration purposes, it is drawn with thickness $2 \varepsilon$ instead of $10 \varepsilon$ )

The limiting functional $E: P C\left(\mathbb{R}^{2} ; \mathcal{Z}\right) \rightarrow[0,+\infty)$ is defined by

$$
E(u)=\int_{J_{u}} \varphi\left(u^{+}(x), u^{-}(x), v_{u}(x)\right) \mathrm{d} \mathcal{H}^{1}(x) .
$$

In view of (2.13), functions in $P C\left(\mathbb{R}^{2} ; \mathcal{Z}\right)$ lie in $S B V$, and therefore $u^{+}, u^{-}$, and $v_{u}$ are well defined. The following statement shows that $E$ can be interpreted as the effective limit of the atomistic energies $E_{\varepsilon}$ in the sense of $\Gamma$-convergence:

Theorem 2.3. ( $\Gamma$-convergence) It holds that $E=\Gamma\left(L_{\mathrm{loc}}^{1}\right)-\lim _{\varepsilon \rightarrow 0} E_{\varepsilon}$; more precisely,

(i) (Г-liminf inequality) For each $u \in P C\left(\mathbb{R}^{2} ; \mathcal{Z}\right)$ and each sequence $\left\{X_{\varepsilon}\right\}_{\varepsilon}$ with $X_{\varepsilon} \rightarrow u$ in $L_{\text {loc }}^{1}\left(\mathbb{R}^{2}\right)$ it holds that

$$
\liminf _{\varepsilon \rightarrow 0} E_{\varepsilon}\left(X_{\varepsilon}\right) \geqq E(u) .
$$

(ii) ( $\Gamma$-limsup inequality) For each $u \in P C\left(\mathbb{R}^{2} ; \mathcal{Z}\right)$ we find configurations $\left\{X_{\varepsilon}\right\}_{\varepsilon}$ such that $X_{\varepsilon} \rightarrow u$ in $L_{\mathrm{loc}}^{1}\left(\mathbb{R}^{2}\right)$ and

$$
\lim _{\varepsilon \rightarrow 0} E_{\varepsilon}\left(X_{\varepsilon}\right)=E(u)
$$

Here and in the sequel, we follow the usual convention that convergence of the continuous parameter $\varepsilon \rightarrow 0$ stands for convergence of arbitrary sequences $\left\{\varepsilon_{k}\right\}_{k}$ with $\varepsilon_{k} \rightarrow 0$ as $k \rightarrow+\infty$. 
Remark 2.4. (Extension to $\left.L^{1}\right)$ Defining $E_{\varepsilon}: L^{1}\left(\mathbb{R}^{2} ; \mathcal{Z}\right) \rightarrow[0,+\infty]$ by

$$
E_{\varepsilon}(u)= \begin{cases}E_{\varepsilon}(X) & \text { if there exists } X \text { such that } u=u_{\varepsilon}^{X}, \\ +\infty & \text { otherwise, }\end{cases}
$$

and extending $E$ to all of $L^{1}\left(\mathbb{R}^{2} ; \mathcal{Z}\right)$ by setting $E(u)=+\infty$ if $u \in L^{1}\left(\mathbb{R}^{2} ; \mathcal{Z}\right) \backslash$ $P C\left(\mathbb{R}^{2} ; \mathcal{Z}\right)$, in view of Theorem 2.1 , this indeed implies $\Gamma\left(L_{\text {loc }}^{1}\right)-\lim _{\varepsilon \rightarrow 0} E_{\varepsilon}=E$.

We close this section by providing properties of the density $\varphi$. To this end, we introduce the function $\varphi_{\text {hex }}: \mathbb{R}^{2} \rightarrow[0,+\infty)$ defined by

$$
\varphi_{\mathrm{hex}}(v)=\frac{2}{\sqrt{3}} \sum_{k=1}^{3}\left|\left\langle\nu, \omega^{k}\right\rangle\right| .
$$

Note that $\varphi_{\text {hex }}$ is a Finsler norm whose unit ball is a regular hexagon in $\mathbb{R}^{2}$ with vertices in $\frac{1}{2} e^{i \pi / 6}\left\{ \pm 1, \pm \omega, \pm \omega^{2}\right\}$, cf. [3,20].

Theorem 2.5. (Properties of $\varphi$ ) Let $\varphi$ be the density given in Proposition 2.2, extended to a function defined on $\mathcal{Z} \times \mathcal{Z} \times \mathbb{R}^{2}$ which is positively 1-homogeneous in the third variable. Then $\varphi$ satisfies the following properties:

(i) (Solid-vacuum energy) There holds $\varphi(z, \mathbf{0}, v)=\varphi(\mathbf{0}, z, v)=\varphi_{\mathrm{hex}}\left(e^{-i \theta} v\right)$ for all $z=(\theta, \tau, 1) \in \mathcal{Z} \backslash\{\mathbf{0}\}$ and $v \in \mathbb{S}^{1}$.

(ii) (Solid-solid energy) There exists a null-set $\mathcal{N}$ in $(\mathcal{Z} \backslash\{\mathbf{0}\})^{2}$ (with respect to its six-dimensional Haar measure) such that for all pairs $\left(z^{+}, z^{-}\right) \in$ $(\mathcal{Z} \backslash\{\mathbf{0}\})^{2} \backslash \mathcal{N}, z^{+} \neq z^{-}$, and $v \in \mathbb{S}^{1}$ that holds

$$
\varphi\left(z^{+}, z^{-}, v\right)=\varphi_{\mathrm{hex}}\left(e^{-i \theta^{+}} v\right)+\varphi_{\mathrm{hex}}\left(e^{-i \theta^{-}} v\right),
$$

and for all $\left(z^{+}, z^{-}\right) \in \mathcal{N}, z^{+} \neq z^{-}$, and $v \in \mathbb{S}^{1}$ it holds that

$\frac{1}{2} \varphi_{\text {hex }}\left(e^{-i \theta^{+}} v\right)+\frac{1}{2} \varphi_{\text {hex }}\left(e^{-i \theta^{-}} v\right) \leqq \varphi\left(z^{+}, z^{-}, v\right)<\varphi_{\text {hex }}\left(e^{-i \theta^{+}} v\right)+\varphi_{\text {hex }}\left(e^{-i \theta^{-}} v\right)$,

where we write $z^{+}=\left(\theta^{+}, \tau^{+}, 1\right)$ and $z^{-}=\left(\theta^{-}, \tau^{-}, 1\right)$.

Moreover, there are exceptional sets $\mathcal{G}_{\mathbb{A}} \subset \mathbb{A}$ of angles and, for each $\theta \in \mathcal{G}_{\mathbb{A}}$, $\mathcal{G}_{\mathbb{T}}(\theta) \subset \mathbb{R}^{2}$ of translation vectors such that $\mathcal{G}_{\mathbb{A}}$ is countable and each $\mathcal{G}_{\mathbb{T}}(\theta)$ is contained in a finite union of spheres, with

$\mathcal{N} \subset\left\{\left(z^{+}, z^{-}\right) \in(\mathcal{Z} \backslash\{\mathbf{0}\})^{2}: \theta^{+}-\theta^{-} \in \mathcal{G}_{\mathbb{A}}, e^{i \theta^{+}} \tau^{+}-e^{i \theta^{-}} \tau^{-} \in \mathcal{G}_{\mathbb{T}}\left(\theta^{+}-\theta^{-}\right)\right\}$.

(iii) (Convexity) The mapping $v \mapsto \varphi\left(z^{+}, z^{-}, v\right)$ is convex for all $z^{+}, z^{-} \in \mathcal{Z}$.

(iv) (Rotational invariance) For all $z^{ \pm}=\left(\theta^{ \pm}, \tau^{ \pm}, 1\right), v \in \mathbb{S}^{1}$, and $\theta \in \mathbb{A}$ it holds that

$$
\varphi\left(\left(\theta^{+}+\theta, \tau^{+}, 1\right),\left(\theta^{-}+\theta, \tau^{-}, 1\right), e^{i \theta} \nu\right)=\varphi\left(\left(\theta^{+}, \tau^{+}, 1\right),\left(\theta^{-} \tau^{-}, 1\right), v\right) .
$$

(v) (Translational invariance) For all $z^{ \pm}=\left(\theta^{ \pm}, \tau^{ \pm}, 1\right), v \in \mathbb{S}^{1}$, and $\tau \in \mathbb{T}$ it holds that

$$
\varphi\left(\left(\theta^{+}, \tau^{+}+e^{-i \theta^{+}} \tau, 1\right),\left(\theta^{-}, \tau^{-}+e^{-i \theta^{-}} \tau, 1\right), v\right)=\varphi\left(\left(\theta^{+}, \tau^{+}, 1\right),\left(\theta^{-} \tau^{-}, 1\right), v\right) .
$$




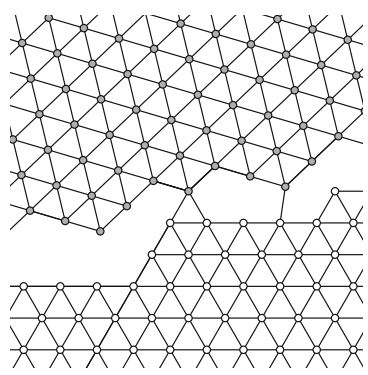

(a)

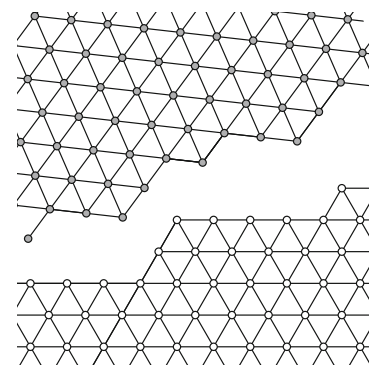

(b)

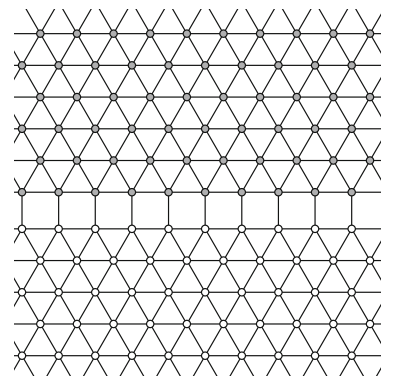

(c)

Fig. 4. Different scenarios of optimal interfaces for a fixed normal $v$ and different lattices $\mathscr{L}\left(z^{ \pm}\right)$. The dark gray and white points form the lattice $\mathscr{L}\left(z^{+}\right)$and the lattice $\mathscr{L}\left(z^{-}\right)$, respectively. Edges are depicted between points of distance 1 . a Two lattices $\mathscr{L}\left(z^{ \pm}\right)$are depicted for which $\varphi$ is less than twice the interaction energy with the vacuum. b We see two lattices $\mathscr{L}\left(z^{ \pm}\right)$for which $\varphi$ is equal to twice the interaction energy with the vacuum. c Two lattices for which the lower bound in Theorem 2.5(ii) is attained

We note that the interaction with vacuum, see property (i), has already been addressed in $[3,20]$. A main novelty of our work lies in the characterization (ii). For explicit choices of the sets $\mathcal{G}_{\mathbb{A}}$ and $\mathcal{G}_{\mathbb{T}}(\theta)$ we refer to (6.2) and the paragraph above Lemma 7.6, respectively. In particular, (ii) states that generically the surface energy between two lattices is if each of the two lattices would interact with vacuum. In this case, the continuum energy $E$ of a function $u=\sum_{j=1}^{\infty} \chi_{G_{j}} z_{j}$ corresponds to the crystalline perimeter of the grains $\left\{G_{j}\right\}_{j}$, induced by $\varphi_{\text {hex }}$. In the non-generic case $\left(z^{+}, z^{-}\right) \in \mathcal{N}$, two lattices $\mathscr{L}\left(z^{+}\right)$and $\mathscr{L}\left(z^{-}\right)$have many touching pairs (that is, pairs of points with distance 1) which reduce the energy (2.3). Optimal interfaces for both cases for a normal vector $v$ are illustrated in Fig. 4. We remark that the exact characterization of $\varphi$ seems to be a difficult issue which is beyond the scope of the present analysis. In fact, counting the number of touching pairs depending on the relative orientation of the two lattices seems to be a non-trivial number theoretic problem, see Remark 2.6 and Fig. 5 below for some details in that direction. We remark that the properties of $\mathcal{G}_{\mathbb{A}}$ and $\mathcal{G}_{\mathbb{T}}(\theta)$ imply that $\mathcal{N}$ is of Hausdorff-dimension at most four. Finally, note that (iv) and (v) express the fact that both the atomistic and the continuum model are frame indifferent.

More precisely, our proof in Lemma 6.2 shows that the non-degeneracy in Theorem 2.5(ii) above can be quantized: for every $\eta>0$ there are only a finite number of differences $\theta$ of lattice rotations and a corresponding finite number of spheres containing the difference of lattice shifts for which

$$
\varphi\left(z^{+}, z^{-}, v\right) \leqq \varphi_{\text {hex }}\left(e^{-i \theta^{+}} v\right)+\varphi_{\text {hex }}\left(e^{-i \theta^{-}} v\right)-\eta .
$$

These numbers only depend on $\eta$. Moreover, we remark that the lower bound provided for $\varphi$ is attained, for example, for $z^{-}=(0,0,1), z^{+}=(0, i, 1)$, and $v=i$, see Fig. 4c. (Consider $X=\{x \in \varepsilon \mathscr{L}(0,0,1):\langle x, i\rangle \leqq 0\} \cup\{x \in \varepsilon \mathscr{L}(0, i, 1):\langle x, i\rangle \geqq$ $\varepsilon\}$ in (2.16).) 


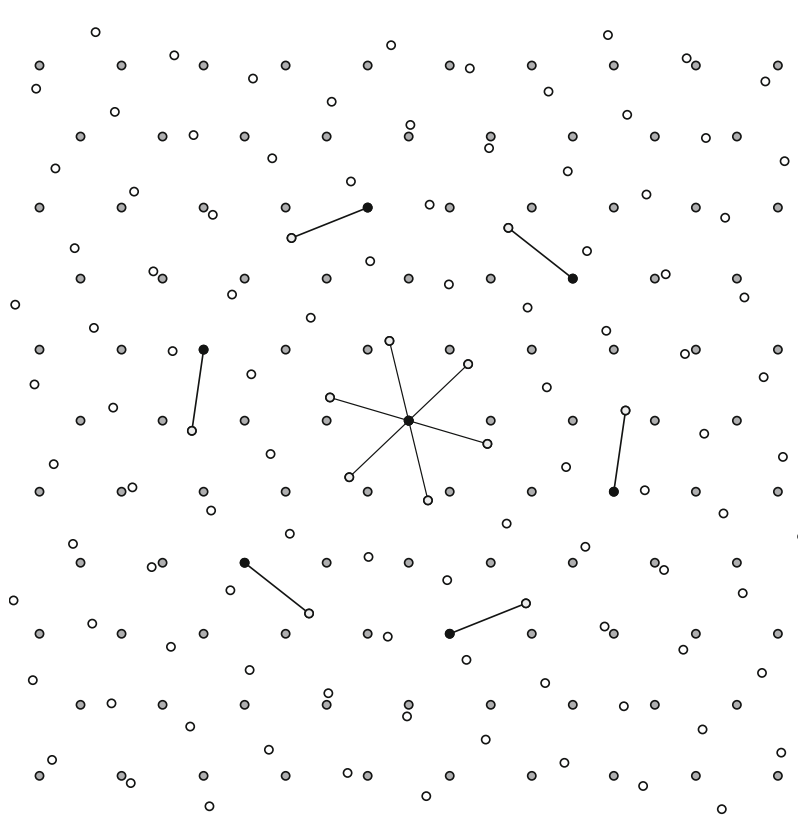

Fig. 5. Two lattices $\mathscr{L}\left(z^{ \pm}\right)$for which $\varphi$ is less than twice the interaction energy with vacuum. The dark gray points form the lattice $\mathscr{L}\left(z^{+}\right)$and the white points the lattice $\mathscr{L}\left(z^{-}\right)$. The black and light gray points are those that are of distance 1 to the other lattice, as emphasized by an edge between them

Remark 2.6. We finally point out that for $\theta^{+}-\theta^{-} \in \mathcal{G}_{\mathbb{A}}, e^{i \theta^{+}} \tau^{+}-e^{i \theta^{-}} \tau^{-} \in$ $\mathcal{G}_{\mathbb{T}}\left(\theta^{+}-\theta^{-}\right)$the calculation of $\varphi$ seems to be a difficult problem. In fact, for $e^{i\left(\theta^{+}-\theta^{-}\right)}=\frac{v_{1}}{v_{2}}$ with $v_{1}, v_{2} \in \mathscr{L}$ and $\left|v_{1}\right|=\left|v_{2}\right|$, depending on the factorization of $v_{1}, v_{2}$ in $\mathscr{L}$, there may be points $(x, y) \in \mathscr{L}\left(z^{+}\right) \times \mathscr{L}\left(z^{-}\right)$such that $x, y \notin$ $\mathscr{L}\left(z^{+}\right) \cap \mathscr{L}\left(z^{-}\right)$and $|x-y|=1$. If this is the case, the relative position of two such atoms is fixed through the prime factors of $v_{1}, v_{2}$, respectively. This leads to two major challenges in the calculation of $\varphi$ : (i) the characterization of points $(x, y) \in \mathscr{L}\left(z^{+}\right) \times \mathscr{L}\left(z^{-}\right)$such that $|x-y|=1$ depending on the relative orientation $e^{i\left(\theta^{+}-\theta^{-}\right)}$of the two lattices seems to be a non-trivial number theoretic problem. (ii) even after the characterization of the set of points $(x, y) \in \mathscr{L}\left(z^{+}\right) \times \mathscr{L}\left(z^{-}\right)$ such that $|x-y|=1$ for different normals $v$ to the interface, it is not always clear if it is energetically convenient to include such points in the construction of the optimal interface due to their relative orientation. Such a situation is illustrated in Fig. 5.

The compactness and $\Gamma$-convergence results will be proved in Section 3 . The properties of several cell formulas related to $\varphi$, which are fundamental for the proofs, are postponed to Sections. 4-7. Finally, the proofs of Proposition 2.2 and Theorem 2.5 are given in Section 7.2. 


\section{Proof of the Main Results}

This section is devoted to the proofs of our main results. We start with some preliminary properties. Then we prove compactness and finally we address the $\Gamma$-convergence result.

\subsection{Preliminaries}

We state and prove some elementary properties of the family $E_{\varepsilon}$. Recall the representation of the energy in (2.3) and the definition of sets in (2.4).

Lemma 3.1. (Properties of $E_{\varepsilon}$ ) Let $\varepsilon>0$ and let $X$ be a configuration with $E_{\varepsilon}(X)<+\infty$. Then it holds that

(i) $E_{\varepsilon}\left(e^{i \theta} X+\tau, e^{i \theta} A+\tau\right)=E_{\varepsilon}(X, A)$ for all $\theta \in[0,2 \pi), \tau \in \mathbb{R}^{2}$, and $A \subset \mathbb{R}^{2}$,

(ii) $E_{\lambda \varepsilon}(\lambda X, \lambda A)=\lambda E_{\varepsilon}(X, A)$ for all $\lambda>0$ and $A \subset \mathbb{R}^{2}$,

(iii) $E_{\varepsilon}(X, A) \leqq E_{\varepsilon}(X, B)$ for all $A \subset B \subset \mathbb{R}^{2}$,

(iv) $E_{\varepsilon}(X, A \cup B)=E_{\varepsilon}(X, B)+E_{\varepsilon}(X, A)$ for all $A, B \subset \mathbb{R}^{2}$ with $A \cap B=\emptyset$,

(v) There exists $C>0$ such that for all $A \subset \mathbb{R}^{2}$ there holds $\#(X \cap A) \leqq$ $C \mathcal{L}^{2}\left((A)_{\varepsilon}\right) / \varepsilon^{2}$.

Proof. Proof of (i): Given $\theta \in[0,2 \pi)$ and $\tau \in \mathbb{R}^{2}$, we define $\tilde{x}=e^{i \theta} x+\tau$ for each $x \in \mathbb{R}^{2}$. The statement follows by noting that $|\tilde{x}-\tilde{y}|=|x-y|$ for all $x, y \in \mathbb{R}^{2}$ and $\tilde{x} \in e^{i \theta} A+\tau$ if and only if $x \in A$. This implies $y \in \mathcal{N}_{\varepsilon}(x)$ if and only if $\tilde{y} \in \mathcal{N}_{\varepsilon}(\tilde{x})$.

Proof of (ii): For $\lambda>0$ and $x \in \mathbb{R}^{2}$, we define $x_{\lambda}=\lambda x$. Clearly, we have $\left|x_{\lambda}-y_{\lambda}\right|=\lambda|x-y|$ for all $x, y \in \mathbb{R}^{2}$ and $x_{\lambda} \in \lambda A$ if and only if $x \in A$. This implies $y_{\lambda} \in \mathcal{N}_{\lambda \varepsilon}\left(x_{\lambda}\right)$ if and only if $y \in \mathcal{N}_{\varepsilon}(x)$.

Proof of (iii): This statement follows from the fact that for all configurations $X$ with finite energy and all $x \in X$ we have $6-\# \mathcal{N}_{\varepsilon}(x) \geqq 0$ by (2.2).

Proof of (iv): This follows from the fact that, if $A \cap B=\emptyset$, each term of the summation on the left hand side occurs also in the right hand side and vice versa.

Proof of (v): Since $X$ is a configuration with finite energy, there holds $|x-y| \geqq \varepsilon$ for all $x, y \in X, x \neq y$. Therefore, $B_{\varepsilon / 2}(x) \cap B_{\varepsilon / 2}(y)=\emptyset$ for all $x, y \in X, x \neq y$. By (2.4), we obtain $\bigcup_{x \in X \cap A} B_{\varepsilon / 2}(x) \subset(A)_{\varepsilon}$ and therefore

$$
\pi \varepsilon^{2} / 4 \#(X \cap A)=\mathcal{L}^{2}\left(\bigcup_{x \in X \cap A} B_{\varepsilon / 2}(x)\right) \leqq \mathcal{L}^{2}\left((A)_{\varepsilon}\right) .
$$

From this the claim follows with $C=4 / \pi$.

The following scaling property will be instrumental:

Lemma 3.2. (Scaling) For $\varepsilon>0$, consider configurations $X_{\varepsilon}$ satisfying $E_{\varepsilon}\left(X_{\varepsilon}\right)<$ $+\infty$ and $\lambda X_{\varepsilon}$ for $\lambda>0$. By $u_{\lambda \varepsilon}^{\lambda}$ and $u_{\varepsilon}$ we denote the functions corresponding to $\lambda X_{\varepsilon}$ and $X_{\varepsilon}$, respectively, as defined in (2.15). Then, there holds

$$
u_{\lambda \varepsilon}^{\lambda}(\lambda x)=u_{\varepsilon}(x) \text { for all } x \in \mathbb{R}^{2} .
$$


Moreover, for each bounded $A \subset \mathbb{R}^{2}$, we have $u_{\lambda \varepsilon}^{\lambda} \rightarrow u\left(\lambda^{-1} \cdot\right)$ in $L^{1}(\lambda A)$ as $\varepsilon \rightarrow 0$ if and only if $u_{\varepsilon} \rightarrow u$ in $L^{1}(A)$.

Proof. We first prove (3.1). To see this, it suffices to note that $x \in X_{\varepsilon}$ if and only if $\lambda x \in \lambda X_{\varepsilon}, \#\left(\mathcal{N}_{\varepsilon}(x) \cap X\right)=6$ if and only if $\#\left(\mathcal{N}_{\lambda \varepsilon}(\lambda x) \cap \lambda X_{\varepsilon}\right)=6$, and $\left(x \cup \mathcal{N}_{\varepsilon}(x)\right) \subset \varepsilon e^{i \theta}(\mathscr{L}+\tau)$ if and only if $\left(\lambda x \cup \mathcal{N}_{\lambda \varepsilon}(\lambda x)\right) \subset \lambda \varepsilon e^{i \theta}(\mathscr{L}+\tau)$ for $\theta \in \mathbb{A}$ and $\tau \in \mathbb{T}$. Therefore, in view of (2.15) and the definition of the Voronoi cells $V_{\varepsilon}^{z}(x)$ below (2.14), (3.1) holds true. The equivalence of the convergence follows by a change of variables: we set $y=\lambda x$ and obtain

$$
\lambda^{2} \int_{A}\left|u_{\varepsilon}(x)-u(x)\right| \mathrm{d} x=\lambda^{2} \int_{A}\left|u_{\lambda \varepsilon}^{\lambda}(\lambda x)-u(x)\right| \mathrm{d} x=\int_{\lambda A}\left|u_{\lambda \varepsilon}^{\lambda}(y)-u\left(\lambda^{-1} y\right)\right| \mathrm{d} y
$$

for every bounded $A \subset \mathbb{R}^{2}$.

\subsection{Compactness}

In this subsection we prove Theorem 2.1. As a preparation, we show the following coercivity property:

Proposition 3.3. (Coercivity) Let $X$ be a configuration with $E_{\varepsilon}(X)<+\infty$ and let $A \subset \mathbb{R}^{2}$ be a Borel set. Then, there exists a universal $C>0$ such that

$$
\mathcal{H}^{1}\left(J_{u} \cap A\right) \leqq C E_{\varepsilon}\left(X,(A)_{\varepsilon}\right),
$$

where $u$ associated to $X$ is given by (2.15) and $(A)_{\varepsilon}$ is defined in (2.4).

Proof. Let $A \subset \mathbb{R}^{2}$ be a Borel set. Consider $X \subset \mathbb{R}^{2}$ with $E_{\varepsilon}(X)<+\infty$. In view of (2.11) and (2.15), the function $u$ associated to $X$ can be written in the form $u=\sum_{j=1}^{\infty} \chi_{G_{j}} z_{j}$ for pairwise distinct $\left\{z_{j}\right\}_{j} \subset \mathcal{Z} \backslash\{\boldsymbol{0}\}$ and pairwise disjoint $\left\{G_{j}\right\}_{j} \subset \mathbb{R}^{2}$. By [2, Remark 4.22] it suffices to check that

$$
\sum_{j=1}^{\infty} \mathcal{H}^{1}\left(\partial^{*} G_{j} \cap A\right) \leqq C E_{\varepsilon}\left(X,(A)_{\varepsilon}\right)
$$

Due to the construction in (2.15), each $G_{j}$ is made of a finite union of regular hexagons with sidelength $\varepsilon / \sqrt{3}$ such that at the center of each such hexagon there is an atom $x \in X$ with $\# \mathcal{N}_{\varepsilon}(x)=6$. If an edge of such a hexagon is contained in $\partial^{*} G_{j}$, then there exists a point $y \in \mathcal{N}_{\varepsilon}(x)$ such that $\# \mathcal{N}_{\varepsilon}(y)<6$, see Fig. 2 . If the intersection of that edge with $A$ is non-empty, then $y \in(A)_{\varepsilon} \cap X$, see (2.1) and (2.4). Note that each such $y$ is selected for at most six different edges of hexagons contained in $\partial G_{j}^{*}$. By (2.3), this yields

$$
\sum_{j \in \mathbb{N}} \mathcal{H}^{1}\left(\partial^{*} G_{j} \cap A\right) \leqq \frac{6}{\sqrt{3}} \varepsilon \#\left\{y \in X \cap(A)_{\varepsilon}: \# \mathcal{N}_{\varepsilon}(y)<6\right\} \leqq \frac{12}{\sqrt{3}} E_{\varepsilon}\left(X,(A)_{\varepsilon}\right),
$$

where we used that each edge of the hexagon has length $\varepsilon / \sqrt{3}$. 
Proof of Theorem 2.1. Let $\left\{X_{\varepsilon}\right\}_{\varepsilon}$ and $\left\{u_{\varepsilon}\right\}_{\varepsilon}$ be given, as defined in (2.15). Recall that $\mathcal{Z}$ can be embedded into $\mathbb{R}^{7}$ and that it is closed and bounded, see (2.9). Therefore, for each $B_{r}, r \in \mathbb{N}$, we can use Proposition 3.3 and a compactness result for piecewise constant functions, see [2, Theorem 4.25], to find a subsequence $\left\{\varepsilon_{k}\right\}_{k}$ and $u^{r} \in P C\left(B_{r} ; \mathcal{Z}\right)$ such that $u_{\varepsilon_{k}} \rightarrow u^{r}$ in measure and thus also in $L^{1}\left(B_{r} ; \mathcal{Z}\right)$. By lower semicontinuity there holds $\mathcal{H}^{1}\left(J_{u^{r}} \cap B_{r}\right) \leqq C$ for a constant independent of $r$. By a diagonal argument, we obtain $u: \mathbb{R}^{2} \rightarrow \mathcal{Z}$ with $u=u^{r}$ on $B_{r}$ for all $r \in \mathbb{N}$ such that $u_{\varepsilon_{k}} \rightarrow u$ in $L_{\text {loc }}^{1}\left(\mathbb{R}^{2} ; \mathcal{Z}\right)$. Clearly, $\mathcal{H}^{1}\left(J_{u}\right)<+\infty$. Thus, to show that $u \in P C\left(\mathbb{R}^{2} ; \mathcal{Z}\right)$, it remains to check that $\mathcal{L}^{2}(\{u \neq \mathbf{0}\})<+\infty$.

Using (3.2) with $A=\mathbb{R}^{2}$, the isoperimetric inequality on $\mathbb{R}^{2}, \mathcal{L}^{2}\left(\left\{u_{\varepsilon_{k}} \neq \mathbf{0}\right\}\right)<$ $+\infty$, and the fact that $\mathcal{L}^{2}(\{u \neq \mathbf{0}\})$ is lower semicontinuous with respect to strong $L_{\text {loc }}^{1}$ convergence, we obtain

$$
\begin{aligned}
\left(\mathcal{L}^{2}(\{u \neq \mathbf{0}\})\right)^{1 / 2} & \leqq \liminf _{k \rightarrow+\infty}\left(\mathcal{L}^{2}\left(\left\{u_{\varepsilon_{k}} \neq \mathbf{0}\right\}\right)\right)^{1 / 2} \leqq \liminf _{k \rightarrow+\infty} C \mathcal{H}^{1}\left(\partial^{*}\left\{u_{\varepsilon_{k}} \neq \mathbf{0}\right\}\right) \\
& \leqq \liminf _{k \rightarrow+\infty} C \mathcal{H}^{1}\left(J_{u_{\varepsilon_{k}}}\right) \leqq \liminf _{k \rightarrow+\infty} C E_{\varepsilon_{k}}\left(X_{\varepsilon_{k}}\right)<+\infty
\end{aligned}
$$

This implies that $u \in P C\left(\mathbb{R}^{2} ; \mathcal{Z}\right)$ and concludes the proof.

\subsection{Lower Bound}

This subsection is devoted to the proof of Theorem 2.3(i). For the proof, it is instrumental to use a different cell formula. In contrast to imposing boundary conditions as in (2.16), we require $L^{1}$-convergence to the function $u_{z^{+}, z^{-}}^{v} \in$ $P C_{\text {loc }}\left(\mathbb{R}^{2} ; \mathcal{Z}\right)$ defined by

$$
u_{z^{+}, z^{-}}^{v}(x)= \begin{cases}z^{+} & \text {if }\langle x, v\rangle \geqq 0, \\ z^{-} & \text {if }\langle x, v\rangle<0,\end{cases}
$$

for $x \in \mathbb{R}^{2}, z^{+}, z^{-} \in \mathcal{Z}$, and $v \in \mathbb{S}^{1}$. More precisely, for $z^{+}, z^{-} \in \mathcal{Z}$ and $v \in \mathbb{S}^{1}$ we introduce

$$
\begin{aligned}
\psi\left(z^{+}, z^{-}, v\right):=\inf & \left\{\liminf _{\varepsilon \rightarrow 0} E_{\varepsilon}\left(X_{\varepsilon}, Q^{v}\left(y_{\varepsilon}\right)\right): y_{\varepsilon} \in \mathbb{R}^{2},\right. \\
& \left.\lim _{\varepsilon \rightarrow 0} \int_{Q^{v}}\left|u_{\varepsilon}\left(x+y_{\varepsilon}\right)-u_{z^{+}, z^{-}}^{v}(x)\right| \mathrm{d} x=0\right\},
\end{aligned}
$$

where $u_{\varepsilon}$ denotes the function associated to $X_{\varepsilon}$, as defined in (2.15). The density $\psi$ is related to $\varphi$ (see (2.16)) in the following way:

Proposition 3.4. (Relation of $\psi$ and $\varphi$ ) For all $z^{+}, z^{-} \in \mathcal{Z}$ and $v \in \mathbb{S}^{1}$ it holds that

$$
\psi\left(z^{+}, z^{-}, v\right) \geqq \varphi\left(z^{+}, z^{-}, v\right) .
$$

We postpone the proof of Proposition 3.4 to Sections $4-7$. It will follow by combining Lemmas 4.1, 7.1, and Proposition 7.2. After a further comment about the definition of $\psi$, we proceed with the proof of the lower bound. 
Remark 3.5. (Varying cubes in the definition of $\psi$ ) We point out that, in contrast to many other cell formulas in the literature, the position of the cubes in (3.5) is not fixed but may vary along the sequence $\varepsilon \rightarrow 0$. This general definition is necessary as the problem is not translation invariant in the variables $z^{ \pm}$, although the discrete energy has such a property, see Lemma 3.1(i). To see this issue, consider a sequence $\left\{X_{\varepsilon}\right\}_{\varepsilon}$ contained in a fixed lattice $X_{\varepsilon} \subset \varepsilon e^{i \theta}(\mathscr{L}+\tau)$. Then, for a fixed translation $\sigma \in \mathbb{R}^{2}$, the shifted configurations $\tilde{X}_{\varepsilon}:=X_{\varepsilon}+\sigma$ are contained in $\varepsilon e^{i \theta}\left(\mathscr{L}+\tau_{\varepsilon}\right)$, where the translation $\tau_{\varepsilon}:=\left(\tau+e^{-i \theta} \sigma / \varepsilon\right)$ (modulo $\mathscr{L}$ ) is in general different from $\tau$ and highly oscillating. This in general implies $\tilde{u}_{\varepsilon} \neq u_{\varepsilon}(\cdot-\sigma)$, where $u_{\varepsilon}$ and $\tilde{u}_{\varepsilon}$ are given in (2.15). This lack of translational invariance is remedied in our approach by minimizing over all possible cell centers. Note that only a posteriori we are able to show that the cell formula $\varphi$ is actually independent of the center, see Proposition 2.2.

Proof of Theorem 2.3(i). Let $\left\{X_{\varepsilon}\right\}_{\varepsilon}$ be a sequence with $X_{\varepsilon} \rightarrow u$ in $L_{\text {loc }}^{1}\left(\mathbb{R}^{2}\right)$ for $u \in P C\left(\mathbb{R}^{2} ; \mathcal{Z}\right)$. Clearly, it suffices to treat the case

$$
\sup _{\varepsilon>0} E_{\varepsilon}\left(X_{\varepsilon}\right)<+\infty .
$$

We proceed in two steps. We first identify a limiting measure associated to the discrete configurations (Step 1). Then, we proceed by a blow-up procedure for the jump part of this measure (Step 2).

Step 1: Identification of a limiting measure. We consider the family of positive measures $\left\{\mu_{\varepsilon}\right\}_{\varepsilon}$ given by

$$
\mu_{\varepsilon}:=\frac{1}{2} \sum_{x \in X} \varepsilon\left(6-\# \mathcal{N}_{\varepsilon}(x)\right) \delta_{x} .
$$

By (2.3) we observe that for all open sets $A \subset \mathbb{R}^{2}$ it holds that

$$
\left|\mu_{\varepsilon}\right|(A)=\mu_{\varepsilon}(A)=E_{\varepsilon}\left(X_{\varepsilon}, A\right)
$$

Therefore, by (3.6) we get $\sup _{\varepsilon>0}\left|\mu_{\varepsilon}\right|\left(\mathbb{R}^{2}\right)<+\infty$. Thus, as $\mathbb{R}^{2}$ is locally compact, up to passing to a subsequence (not relabeled), there exists a positive finite Radon measure $\mu$ such that

$$
\mu_{\varepsilon} \stackrel{*}{\rightarrow} \mu
$$

By the Radon-Nykodym Theorem we may decompose $\mu$ into two mutually singular non-negative measures

$$
\mu=\left.\xi \mathcal{H}^{1}\right|_{J_{u}}+\mu_{s}
$$

The main point is to prove

$$
\xi\left(x_{0}\right) \geqq \psi\left(z^{+}, z^{-}, v\right) \text { for } \mathcal{H}^{1} \text {-almost every } x_{0} \in J_{u},
$$

where $z^{+}$and $z^{-}$denote the one-sided limits of $u$ at $x_{0}$ and $v$ denotes the corresponding normal. (For notational convenience, the explicit dependence on $u$ is 
omitted.) Once this is shown, the statement follows from (2.17), (3.7), (3.8), and Proposition 3.4. In fact,

$$
\begin{aligned}
\liminf _{\varepsilon \rightarrow 0} E_{\varepsilon}\left(X_{\varepsilon}\right) & =\liminf _{\varepsilon \rightarrow 0} \mu_{\varepsilon}\left(\mathbb{R}^{2}\right) \geqq \mu\left(\mathbb{R}^{2}\right) \geqq \int_{J_{u}} \xi \mathrm{d} \mathcal{H}^{1} \geqq \int_{J_{u}} \varphi\left(z^{+}, z^{-}, v\right) \mathrm{d} \mathcal{H}^{1} \\
& =E(u) .
\end{aligned}
$$

Step 2: Blow-up argument. It remains to prove (3.9). By the properties of $S B V$ functions and Radon measures we know that for $\mathcal{H}^{1}$-almost every $x_{0} \in J_{u}$ it holds that

$$
\begin{aligned}
& \text { (a) } \lim _{\rho \rightarrow 0} \frac{1}{\rho^{2}} \int_{Q_{\rho}^{v}\left(x_{0}\right)}\left|u(x)-u_{z^{+}, z^{-}}^{v}\left(x-x_{0}\right)\right| \mathrm{d} x=0, \\
& \text { (b) } \lim _{\rho \rightarrow 0} \frac{1}{\rho} \mathcal{H}^{1}\left(J_{u} \cap Q_{\rho}^{v}\left(x_{0}\right)\right)=1, \\
& \text { (c) } \xi\left(x_{0}\right)=\lim _{\rho \rightarrow 0} \frac{\mu\left(Q_{\rho}^{v}\left(x_{0}\right)\right)}{\mathcal{H}^{1}\left(J_{u} \cap Q_{\rho}^{v}\left(x_{0}\right)\right)} ;
\end{aligned}
$$

see, for example, [2, Theorem 2.63, Theorem 3.78, and Remark 3.79]. Here, $u_{z^{+}, z^{-}}$ is defined in (3.4). It suffices to prove (3.9) for all $x_{0} \in J_{u}$ such that (a)-(c) hold. We fix $\rho_{n} \rightarrow 0$ such that $|\mu|\left(\partial Q_{\rho_{n}}^{v}\left(x_{0}\right)\right)=0$ for all $n \in \mathbb{N}$. By (3.7), (3.8), (b), (c), and the Portmanteu Theorem, we get

$$
\begin{aligned}
\xi\left(x_{0}\right) & =\lim _{\rho \rightarrow 0} \frac{\mu\left(Q_{\rho}^{v}\left(x_{0}\right)\right)}{\mathcal{H}^{1}\left(J_{u} \cap Q_{\rho}^{v}\left(x_{0}\right)\right)}=\lim _{\rho \rightarrow 0} \frac{\mu\left(Q_{\rho}^{v}\left(x_{0}\right)\right)}{\rho}=\lim _{n \rightarrow+\infty} \frac{1}{\rho_{n}} \lim _{\varepsilon \rightarrow 0} \mu_{\varepsilon}\left(Q_{\rho_{n}}^{v}\left(x_{0}\right)\right) \\
& =\lim _{n \rightarrow+\infty} \frac{1}{\rho_{n}} \lim _{\varepsilon \rightarrow 0} E_{\varepsilon}\left(X_{\varepsilon}, Q_{\rho_{n}}^{v}\left(x_{0}\right)\right) .
\end{aligned}
$$

We introduce the configuration $X_{\varepsilon}^{n}:=\rho_{n}^{-1} X_{\varepsilon}$ and obtain by Lemma 3.1(ii) (for $\left.\lambda=1 / \rho_{n}\right)$

$$
\xi\left(x_{0}\right)=\lim _{n \rightarrow+\infty} \lim _{\varepsilon \rightarrow 0} E_{\varepsilon / \rho_{n}}\left(X_{\varepsilon}^{n}, Q^{v}\left(\rho_{n}^{-1} x_{0}\right)\right) .
$$

Since $X_{\varepsilon} \rightarrow u$ in $L_{\text {loc }}^{1}\left(\mathbb{R}^{2}\right)$, we obtain by definition that $u_{\varepsilon} \rightarrow u$ in $L_{\text {loc }}^{1}\left(\mathbb{R}^{2}\right)$, see the end of Section 2.2. By $u_{\varepsilon}^{n}$ we denote the function corresponding to $X_{\varepsilon}^{n}$. By (3.1) we have $u_{\varepsilon}^{n}(x)=u_{\varepsilon}\left(\rho_{n} x\right)$ for all $x \in \mathbb{R}^{2}$. In particular, Lemma 3.2 yields $u_{\varepsilon}^{n} \rightarrow u^{n}$ on $Q^{v}\left(\rho_{n}^{-1} x_{0}\right)$, where $u^{n}(x):=u\left(\rho_{n} x\right)$ for $x \in \mathbb{R}^{2}$. By (a), change of variables, and the fact that $u^{n}\left(x+\rho_{n}^{-1} x_{0}\right)=u\left(x_{0}+\rho_{n} x\right)$ as well as $u_{z^{+}, z^{-}}^{v}(x)=u_{z^{+}, z^{-}}^{v}\left(\rho_{n} x\right)$ for $x \in \mathbb{R}^{2}$, we also get that

$$
\begin{aligned}
& \lim _{n \rightarrow+\infty} \int_{Q^{v}}\left|u^{n}\left(x+\rho_{n}^{-1} x_{0}\right)-u_{z^{+}, z^{-}}^{v}(x)\right| \mathrm{d} x \\
& =\lim _{n \rightarrow+\infty} \frac{1}{\rho_{n}^{2}} \int_{Q_{\rho_{n}}^{v}\left(x_{0}\right)}\left|u(x)-u_{z^{+}, z^{-}}\left(x-x_{0}\right)\right| \mathrm{d} x=0 .
\end{aligned}
$$

Therefore, by recalling (3.10) and $u_{\varepsilon}^{n} \rightarrow u^{n}$ on $Q^{v}\left(\rho_{n}^{-1} x_{0}\right)$, by using a standard diagonal argument, we find an infinitesimal sequence $\{\varepsilon(n)\}_{n}$ such that for $X^{n}:=$ $X_{\varepsilon(n)}^{n}$ and $u^{n}:=u_{\varepsilon(n)}^{n}$ we have

$$
\xi\left(x_{0}\right)=\lim _{n \rightarrow+\infty} E_{\varepsilon_{n}}\left(X^{n}, Q^{v}\left(y^{n}\right)\right),
$$


and

$$
\lim _{n \rightarrow+\infty} \int_{Q^{v}}\left|u^{n}\left(x+y^{n}\right)-u_{z^{+}, z^{-}}^{v}(x)\right| \mathrm{d} x=0,
$$

where $\varepsilon_{n}=\varepsilon(n) / \rho_{n}$ and $y^{n}=\rho_{n}^{-1} x_{0}$. Since the sequence is admissible in (3.5), (3.11) implies $\xi\left(x_{0}\right) \geqq \psi\left(z^{+}, z^{-}, v\right)$. This shows (3.9) and concludes the proof.

\subsection{Upper Bound}

This subsection is devoted to the proof of Theorem 2.3(ii). The following density result will be instrumental:

Lemma 3.6. Let $u \in P C\left(\mathbb{R}^{2} ; \mathcal{Z}\right)$. Then there exists a sequence $\left(u_{n}\right)_{n} \subset P C\left(\mathbb{R}^{2} ; \mathcal{Z}\right)$ with $u_{n} \rightarrow u$ in $L^{1}\left(\mathbb{R}^{2}\right)$ and $\lim \sup _{n \rightarrow+\infty} E\left(u_{n}\right) \leqq E(u)$ such that each $u_{n}$ attains only finitely many values and has polygonal jump set, that is, $J_{u_{n}}$ consists of finitely many segments.

Proof. Consider $u \in P C\left(\mathbb{R}^{2} ; \mathcal{Z}\right)$. We proceed in three steps. We first show that $u$ can be approximated by functions with finite support (Step 1). Then, we approximate with functions attaining only finitely many values (Step 2) and finally show that the jump set can be approximated by a finite number of segments (Step 3). Note that it suffices to show that for each $\delta>0$ there exists a function $u_{\delta}$ with the desired properties satisfying

$$
E\left(u_{\delta}\right) \leqq E(u)+\delta \quad \text { and } \quad\left\|u-u_{\delta}\right\|_{L^{1}\left(\mathbb{R}^{2}\right)} \leqq \delta .
$$

We prove (3.12) up to the multiplication with a uniform constant $C>0$ that is independent of $\delta$. Replacing $u_{\delta}$ with $u_{\delta / C}$ then yields the result.

Step 1: Reduction to finite support. We show that for every $u \in P C\left(\mathbb{R}^{2} ; \mathcal{Z}\right)$ and for every $\delta>0$ there exist $R>0$ and $u_{\delta} \in P C\left(\mathbb{R}^{2} ; \mathcal{Z}\right)$ such that $(3.12)$ is satisfied and it holds that

$$
\left\{u_{\delta} \neq \mathbf{0}\right\} \subset B_{R} .
$$

To this end, fix $\delta>0$. Since there holds $\mathcal{L}^{2}(\{u \neq \mathbf{0}\})<+\infty$, we can choose $R^{\prime}>0$ such that

$$
\mathcal{L}^{2}\left(\{u \neq \mathbf{0}\} \cap\left(\mathbb{R}^{2} \backslash B_{R^{\prime}}\right)\right) \leqq \delta .
$$

By the coarea formula and the previous inequality, we can select $R \in\left(R^{\prime}, R^{\prime}+1\right)$ such that

$$
\begin{aligned}
\mathcal{H}^{1}\left(\{u \neq \mathbf{0}\} \cap \partial B_{R}\right) & \leqq \mathcal{L}^{2}\left(\{u \neq \mathbf{0}\} \cap\left(B_{R^{\prime}+1} \backslash B_{R^{\prime}}\right)\right) \\
& \leqq \mathcal{L}^{2}\left(\{u \neq \mathbf{0}\} \cap\left(\mathbb{R}^{2} \backslash B_{R^{\prime}}\right)\right) \leqq \delta .
\end{aligned}
$$

Define $u_{\delta} \in P C\left(\mathbb{R}^{2} ; \mathcal{Z}\right)$ by $u_{\delta}=u \chi_{B_{R}}$. Then clearly (3.13) holds. We choose the orientation of $v_{u_{\delta}}(x)$ for $x \in J_{u} \cap \partial B_{R}$ such that $u_{\delta}^{+}$coincides with the trace 
of $u$ from the interior of $B_{R}$. As $\varphi(z, \mathbf{0}, v) \leqq C$ for all $z \in \mathcal{Z}$ and $v \in \mathbb{S}^{1}$ by Theorem 2.5(i), we use (3.15) to get

$$
\begin{aligned}
E\left(u_{\delta}\right) & =\int_{B_{R} \cap J_{u_{\delta}}} \varphi\left(u_{\delta}^{+}, u_{\delta}^{-}, v_{u_{\delta}}\right) \mathrm{d} \mathcal{H}^{1}+\int_{\partial B_{R} \cap\{u \neq \mathbf{0}\}} \varphi\left(u_{\delta}^{+}, \mathbf{0}, v_{u_{\delta}}\right) \mathrm{d} \mathcal{H}^{1} \\
& \leqq \int_{B_{R} \cap J_{u}} \varphi\left(u^{+}, u^{-}, v_{u}\right) \mathrm{d} \mathcal{H}^{1}+C \mathcal{H}^{1}\left(\{u \neq \mathbf{0}\} \cap \partial B_{R}\right) \leqq E(u)+C \delta .
\end{aligned}
$$

This implies the first inequality of (3.12). To see the second inequality of (3.12), note that $|z| \leqq C$ for all $z \in \mathcal{Z}$ and therefore by (3.14)

$$
\left\|u_{\delta}-u\right\|_{L^{1}\left(\mathbb{R}^{2}\right)}=\left\|u_{\delta}-u\right\|_{L^{1}\left(\mathbb{R}^{2} \backslash B_{R}\right)} \leqq C \mathcal{L}^{2}\left(\{u \neq \mathbf{0}\} \cap\left(\mathbb{R}^{2} \backslash B_{R^{\prime}}\right)\right) \leqq C \delta .
$$

Step 2: Reduction to functions attaining finitely many values. Consider $u \in P C\left(\mathbb{R}^{2} ; \mathcal{Z}\right)$. By Step 1 we may assume that (3.13) holds for some $R>0$, that is, $\{u \neq \mathbf{0}\} \subset B_{R}$. For each $\delta>0$, we prove that there exists $u_{\delta} \in P C\left(\mathbb{R}^{2} ; \mathcal{Z}\right)$ such that (3.12) holds and $u_{\delta}$ attains only finitely many values. Recall by (2.11) that $u$ can be written in the form $u=\sum_{j=1}^{\infty} \chi_{G_{j}} z_{j}$ for pairwise distinct $\left\{z_{j}\right\}_{j} \subset \mathcal{Z} \backslash\{\boldsymbol{0}\}$ and pairwise disjoint $\left\{G_{j}\right\}_{j} \subset \mathbb{R}^{2}$. In view of (2.12), we can choose $J_{\delta} \in \mathbb{N}$ sufficiently large such that

$$
\sum_{j=J_{\delta}+1}^{\infty} \mathcal{H}^{1}\left(\partial^{*} G_{j}\right) \leqq \delta / R .
$$

Note that $G_{j} \subset B_{R}$ for all $j \in \mathbb{N}$ since $\{u \neq \mathbf{0}\} \subset B_{R}$. Due to the isoperimetric inequality on $B_{R}$ along with $\mathcal{L}^{2}\left(G_{j}\right) \leqq \mathcal{L}^{2}\left(B_{R}\right)=\pi R^{2}$ for all $j \in \mathbb{N}$, we obtain

$$
\sum_{j=J_{\delta}+1}^{\infty} \mathcal{L}^{2}\left(G_{j}\right) \leqq \sqrt{\pi R^{2}} \sum_{j=J_{\delta}+1}^{\infty}\left(\mathcal{L}^{2}\left(G_{j}\right)\right)^{1 / 2} \leqq C R \sum_{j=J_{\delta}+1}^{\infty} \mathcal{H}^{1}\left(\partial^{*} G_{j}\right) \leqq C \delta
$$

where $C>0$ is a universal constant. Now we define

$$
u_{\delta}:=\left\{\begin{array}{l}
u \text { in } \bigcup_{j=1}^{J_{\delta}} G_{j}, \\
\mathbf{0} \text { otherwise. }
\end{array}\right.
$$

Then, by (3.17) and $\|u\|_{\infty} \leqq C$ we get $\left\|u_{\delta}-u\right\|_{L^{1}\left(\mathbb{R}^{2}\right)}=\left\|u_{\delta}-u\right\|_{L^{1}\left(B_{R}\right)} \leqq C \delta$. Moreover, setting for brevity $\Gamma:=\bigcup_{j=J_{\delta}+1}^{\infty} \partial^{*} G_{j}$ we obtain, by (3.16), that

$$
\begin{aligned}
E\left(u_{\delta}\right)= & \int_{J_{u_{\delta}}} \varphi\left(u_{\delta}^{+}, u_{\delta}^{-}, v_{u_{\delta}}\right) \mathrm{d} \mathcal{H}^{1}=\int_{J_{u_{\delta}} \cap \Gamma} \varphi\left(u_{\delta}^{+}, u_{\delta}^{-}, v_{u_{\delta}}\right) \mathrm{d} \mathcal{H}^{1} \\
& +\int_{J_{u_{\delta} \backslash \Gamma}} \varphi\left(u_{\delta}^{+}, u_{\delta}^{-}, v_{u_{\delta}}\right) \mathrm{d} \mathcal{H}^{1} \\
\leqq & C \sum_{j=J_{\delta}+1}^{\infty} \mathcal{H}^{1}\left(\partial^{*} G_{j}\right)+E(u) \leqq C \delta+E(u),
\end{aligned}
$$




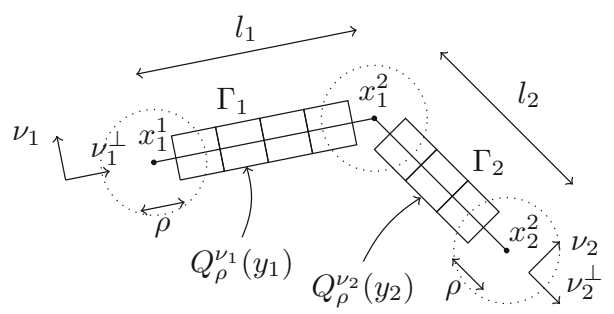

Fig. 6. The construction for the $\Gamma$-lim sup in the case where the jump set is polyhedral: The part $\Gamma_{1} \cup \Gamma_{2}$ of the jump set is shown. Here, $x_{1}^{2}$ equals $x_{2}^{1}$. The region $(M)_{\delta}$ is shown as the dotted circles around the points in $M$. Also the cubes used in the construction to cover the segments $\Gamma_{1}$ and $\Gamma_{2}$ are indicated

where we have used $\varphi\left(z_{1}, z_{2}, v\right) \leqq C$ for all $z_{1}, z_{2} \in \mathcal{Z}$ and $v \in \mathbb{S}^{1}$. Therefore, (3.12) holds, and Step 2 is concluded.

Step 3: Reduction to polyhedral jump sets. Consider $u \in P C\left(\mathbb{R}^{2} ; \mathcal{Z}\right)$. By Steps $1-2$ we can assume that $u$ attains only finitely many values, and its support is contained in $B_{R}$. By Theorem 2.5(iii) we get that the mapping $v \mapsto \varphi\left(z_{1}, z_{2}, v\right)$ is convex and thus continuous for all $z_{1}, z_{2} \in \mathcal{Z}$. Therefore, by [9, Theorem 2.1 and Corollary 2.4] (with $\Omega=B_{R}$ and $\mathcal{Z}$ being the range of $u$ ) we obtain a function $u_{\delta} \in P C\left(\mathbb{R}^{2} ; \mathcal{Z}\right.$ ) with polyhedral jump set such that (3.12) is satisfied. This concludes the proof.

We are now in a position to prove Theorem 2.3(ii).

Proof of Theorem 2.3(ii). By Lemma 3.6 and a general density argument in the theory of $\Gamma$-convergence (see [8, Remark 1.29]), it suffices to construct recovery sequences for $u \in P C\left(\mathbb{R}^{2} ; \mathcal{Z}\right)$ such that $u$ attains only finitely many values, and $u$ has a polygonal jump set. Our goal is to prove that there exists $\left\{X_{\varepsilon}\right\}_{\varepsilon}$ such that $X_{\varepsilon} \rightarrow u$ in $L_{\text {loc }}^{1}\left(\mathbb{R}^{2}\right)$ and $\lim \sup _{\varepsilon \rightarrow 0} E_{\varepsilon}\left(X_{\varepsilon}\right) \leqq E(u)$.

Let $J_{u}=\bigcup_{i=1}^{N} \Gamma_{i}=\bigcup_{i=1}^{N}\left[x_{i}^{1} ; x_{i}^{2}\right]$, where the sets $\Gamma_{i}$ are line segments between the points $x_{i}^{1}$ and $x_{i}^{2}$, defined in (2.5), with length $l_{i}$, orientation $v_{i}^{\perp}$, and normal $v_{i}$. We can assume that the traces $\left(u^{+}, u^{-}\right)=\left(u_{i}^{+}, u_{i}^{-}\right)$are constant along each line segment, and that two segments $\Gamma_{i}$ and $\Gamma_{j}$ intersect at most at endpoints of $\Gamma_{i}$ and $\Gamma_{j}$. Denote by $M$ the collection of points where at least two of such line segments meet. Fix $0<\delta<\frac{1}{3} \min \{|x-y|: x, y \in M, x \neq y\}$ and choose $\rho \in(0, \delta)$ small enough such that

$$
\rho<\frac{1}{\sqrt{2}} \operatorname{dist}\left(\Gamma_{i} \backslash\left(B_{\delta}\left(x_{i}^{1}\right) \cup B_{\delta}\left(x_{i}^{2}\right)\right), \Gamma_{j} \backslash\left(B_{\delta}\left(x_{j}^{1}\right) \cup B_{\delta}\left(x_{j}^{2}\right)\right)\right) \quad \text { for all } i \neq j .
$$

This choice of $\rho$ implies that $Q_{\rho}^{v}\left(x_{1}\right) \cap Q_{\rho}^{v}\left(x_{2}\right)=\emptyset$ for all $x_{1} \in \Gamma_{i} \backslash\left(B_{\delta}\left(x_{i}^{1}\right) \cup\right.$ $\left.B_{\delta}\left(x_{i}^{2}\right)\right)$ and $x_{2} \in \Gamma_{j} \backslash\left(B_{\delta}\left(x_{j}^{1}\right) \cup B_{\delta}\left(x_{j}^{2}\right)\right), i \neq j$. As the traces $\left(u^{+}, u^{-}\right)$are constant on $\Gamma_{i}$, it holds that

$$
\int_{\Gamma_{i}} \varphi\left(u^{+}, u^{-}, v_{u}\right) \mathrm{d} \mathcal{H}^{1}=l_{i} \varphi\left(u_{i}^{+}, u_{i}^{-}, v_{i}\right) \text { for all } i \in\{1, \ldots, N\} .
$$


We define

$$
P_{i}^{\rho}=\left\{x_{i}^{1}+k \rho v_{i}^{\perp}: k \in \mathbb{N}, 0 \leqq k \leqq\left\lfloor l_{i} / \rho\right\rfloor\right\}, \quad \Gamma_{i}^{\rho}=\bigcup_{x \in P_{i}^{\rho}} Q_{\rho}^{v}(x), \quad \Gamma_{\rho}=\bigcup_{i=1}^{N} \Gamma_{i}^{\rho}
$$

as well as (recall (2.7))

$H^{\varepsilon}=\bigcup_{i=1}^{N} \bigcup_{x \in P_{i}^{\rho}}\left(x+\partial_{\varepsilon}^{H} Q_{\rho}^{v}\right)$, where $\partial_{\varepsilon}^{H} Q_{\rho}^{v}:=\overline{Q_{\rho+10 \varepsilon}^{v} \backslash Q_{\rho-10 \varepsilon}^{v} \backslash} \backslash\left(\partial_{\varepsilon}^{+} Q_{\rho}^{v} \cup \partial_{\varepsilon}^{-} Q_{\rho}^{v}\right)$.

In view of Proposition 2.2, we can choose $\varepsilon=\varepsilon(\rho, \delta)>0$ sufficiently small such that, for each $x \in P_{i}^{\rho}$, we can choose a configuration $X_{\varepsilon}^{x} \subset \mathbb{R}^{2}$ satisfying $X_{\varepsilon}^{x}=\varepsilon \mathscr{L}\left(u_{i}^{ \pm}\right)$on $\partial_{\varepsilon}^{ \pm} Q_{\rho}^{v}(x)$ and

$$
E_{\varepsilon}\left(X_{\varepsilon}^{x}, Q_{\rho}^{v}(x)\right) \leqq \rho \varphi\left(u_{i}^{+}, u_{i}^{-}, v_{i}\right)+\delta \rho / l_{i} .
$$

We introduce the configuration

$$
X_{\varepsilon}^{\delta}= \begin{cases}X_{\varepsilon}^{x} & \text { in } Q_{\rho}^{v}(x) \backslash\left((M)_{\delta} \cup H^{\varepsilon}\right), \text { for } x \in P_{i}^{\rho} \text { for some } i \in\{1, \ldots, N\}, \\ \mathscr{L}(z) & \text { in }\{u=z\} \backslash\left((M)_{\delta} \cup \Gamma_{\rho}\right) \text { for } z \in \operatorname{Im}(u), \\ \varnothing & \text { in }(M)_{\delta} \cup H^{\varepsilon}\end{cases}
$$

see Fig. 6 for an illustration. Here, $(M)_{\delta}$ denotes the $\delta$-neighborhood of $M$, see (2.4), and $\operatorname{Im}(u)$ denotes the image of $u$. The set $H^{\varepsilon}$ is introduced in order to ensure that $E_{\varepsilon}\left(X_{\varepsilon}^{\delta}\right)<+\infty$ since atoms in $H^{\varepsilon}$ of two adjacent cubes could violate the constraint of having at least distance $\varepsilon$. Indeed, by $X_{\varepsilon}^{\delta}=\emptyset$ on $(M)_{\delta} \cup H^{\varepsilon}$ and the boundary conditions of $X_{\varepsilon}^{x}$, we get $|x-y| \geqq \varepsilon$ for all $x, y \in X_{\varepsilon}^{\delta}, x \neq y$, and therefore $E_{\varepsilon}\left(X_{\varepsilon}^{\delta}\right)<+\infty$. We have $\# \mathcal{N}_{\varepsilon}(x)=6$ for each atom $x \in X_{\varepsilon}^{\delta} \backslash\left((M)_{\delta+\varepsilon} \cup \Gamma_{\rho}\right)$. To see this, we take the boundary conditions of $X_{\varepsilon}^{x}$ and the choice of $\rho$ in (3.18) into account. By (2.3) this implies

$$
E_{\varepsilon}\left(X_{\varepsilon}^{\delta}, \mathbb{R}^{2} \backslash\left((M)_{\delta+\varepsilon} \cup \Gamma_{\rho}\right)\right)=0 .
$$

Therefore, it remains to account for the energy contribution inside the cubes $Q_{\rho}^{v}(x)$, $x \in P_{i}^{\rho}$, and the set $(M)_{\delta+\varepsilon}$. First, note that for $\bar{x} \in M$ we have that

$$
\#\left(X_{\varepsilon}^{\delta} \cap B_{\delta+\varepsilon}(\bar{x})\right) \leqq C \delta / \varepsilon
$$

In fact, $(M)_{\delta} \cap X_{\varepsilon}^{\delta}=\emptyset$ by definition and thus $X_{\varepsilon}^{\delta} \cap B_{\delta}(\bar{x})=\emptyset$. As $E_{\varepsilon}\left(X_{\varepsilon}^{\delta}\right)<+\infty$, by Lemma 3.1(v) and a simple computation we get $\#\left(X_{\varepsilon}^{\delta} \cap\left(B_{\delta+\varepsilon}(\bar{x}) \backslash B_{\delta}(\bar{x})\right)\right) \leqq$ $C \varepsilon^{-2} \mathcal{L}^{2}\left(B_{\delta+2 \varepsilon}(\bar{x}) \backslash B_{\delta-\varepsilon}(\bar{x})\right) \leqq C \delta / \varepsilon$ for a universal constant $C>0$. This yields (3.22) and then by (2.3) we get

$$
E_{\varepsilon}\left(X_{\varepsilon}^{\delta},(M)_{\delta+\varepsilon}\right) \leqq C \delta,
$$

where $C$ depends also on \#M. By definition of $X_{\varepsilon}^{\delta}$, for $x \in P_{i}^{\rho}$ we have that $X_{\varepsilon}^{\delta}=$ $X_{\varepsilon}^{x}$ in $Q_{\rho+\varepsilon}^{v}(x) \backslash\left(H^{\varepsilon} \cup(M)_{\delta}\right)$. As $E_{\varepsilon}\left(X_{\varepsilon}^{\delta}\right)<+\infty$, we can employ Lemma 3.1(v) 
to deduce that $\#\left(X_{\varepsilon}^{\delta} \cap\left(H^{\varepsilon}\right)_{\varepsilon} \cap Q_{\rho}^{\nu}(x)\right) \leqq \varepsilon^{-2} C \mathcal{L}^{2}\left(\left(H^{\varepsilon} \cap Q_{\rho}^{\nu}(x)\right)_{2 \varepsilon}\right) \leqq C$. Hence, by (2.3) we obtain

$$
E_{\varepsilon}\left(X_{\varepsilon}^{\delta}, Q_{\rho}^{v}(x)\right) \leqq E_{\varepsilon}\left(X_{\varepsilon}^{x}, Q_{\rho}^{v}(x)\right)+C \varepsilon
$$

for all $x \in P_{i}^{\rho}$ such that $\operatorname{dist}\left(Q_{\rho}^{v}(x),(M)_{\delta}\right) \geqq \varepsilon$. On the other hand, for $x \in P_{i}^{\rho}$ such that $\operatorname{dist}\left(Q_{\rho}^{\nu}(x),(M)_{\delta}\right)<\varepsilon$, we use the estimate in (3.22) with $\bar{x} \in M$ such that $\operatorname{dist}\left(Q_{\rho}^{v}(x),(M)_{\delta}\right)=\operatorname{dist}\left(Q_{\rho}^{v}(x), B_{\delta}(\bar{x})\right)$ (and so $\operatorname{dist}\left(Q_{\rho}^{v}(x),(M \backslash\{\bar{x}\})_{\delta}\right)>\varepsilon$ ) and obtain

$$
E_{\varepsilon}\left(X_{\varepsilon}^{\delta}, Q_{\rho}^{v}(x)\right) \leqq E_{\varepsilon}\left(X_{\varepsilon}^{x}, Q_{\rho}^{v}(x)\right)+C(\varepsilon+\delta)
$$

Consequently, using (3.20), (3.24)-(3.25), and Lemma 3.1(iii), we obtain

$$
\begin{aligned}
& \sum_{x \in P_{i}^{\rho}} E_{\varepsilon}\left(X_{\varepsilon}^{\delta}, Q_{\rho}^{v}(x) \backslash(M)_{\delta+\varepsilon}\right) \leqq \sum_{x \in \tilde{P}_{i}^{\rho}} E_{\varepsilon}\left(X_{\varepsilon}^{\delta}, Q_{\rho}^{v}(x)\right) \\
& \quad \leqq \rho\left\lfloor l_{i} / \rho\right\rfloor \varphi\left(u_{i}^{+}, u_{i}^{-}, v_{i}\right)+C \delta+C \varepsilon / \rho,
\end{aligned}
$$

where we have set $\tilde{P}_{i}^{\rho}=\left\{x \in P_{i}^{\rho}: Q_{\rho}^{\nu}(x) \not \subset(M)_{\delta+\varepsilon}\right\}$. Here, $C$ depends on $N$ and $\# M$, but is independent of $\varepsilon, \delta$, and $\rho$. Thus, by choosing $\varepsilon$ small enough with respect to $\rho$ (that is, with respect to $\delta$ ) we get by $(3.19)$ that

$$
\begin{aligned}
\sum_{x \in P_{i}^{\rho}} E_{\varepsilon}\left(X_{\varepsilon}^{\delta}, Q_{\rho}^{v}(x) \backslash(M)_{\delta+\varepsilon}\right) & \leqq l_{i} \varphi\left(u_{i}^{+}, u_{i}^{-}, v_{i}\right)+C \delta \\
& =\int_{\Gamma_{i} \cap J_{u}} \varphi\left(u^{+}, u^{-}, v_{u}\right) \mathrm{d} \mathcal{H}^{1}+C \delta .
\end{aligned}
$$

Now, by Lemma 3.1(iv), (3.21), (3.23), and (3.26) we conclude

$$
\begin{aligned}
E_{\varepsilon}\left(X_{\varepsilon}^{\delta}\right) \leqq & \sum_{i=1}^{N} \sum_{x \in P_{i}^{\rho}} E_{\varepsilon}\left(X_{\varepsilon}^{\delta}, Q_{\rho}^{v}(x) \backslash(M)_{\delta+\varepsilon}\right)+E_{\varepsilon}\left(X_{\varepsilon}^{\delta},(M)_{\delta+\varepsilon}\right) \\
& +E_{\varepsilon}\left(X_{\varepsilon}^{\delta}, \mathbb{R}^{2} \backslash\left((M)_{\delta+\varepsilon} \cup \Gamma_{\rho}\right)\right) \\
\leqq & \sum_{i=1}^{N} \int_{\Gamma_{i} \cap J_{u}} \varphi\left(u^{+}, u^{-}, v_{u}\right) \mathrm{d} \mathcal{H}^{1}+C N \delta=\int_{J_{u}} \varphi\left(u^{+}, u^{-}, v_{u}\right) \mathrm{d} \mathcal{H}^{1}+C N \delta
\end{aligned}
$$

By choosing $\delta=\delta(\varepsilon) \rightarrow 0$ sufficiently slowly as $\varepsilon \rightarrow 0$ we obtain $X_{\varepsilon}^{\delta(\varepsilon)} \rightarrow u$ in $L_{\text {loc }}^{1}\left(\mathbb{R}^{2}\right)$ (see Section 2.2 for the definition of this convergence) and

$$
\limsup _{\varepsilon \rightarrow 0} E_{\varepsilon}\left(X_{\varepsilon}^{\delta(\varepsilon)}\right) \leqq \int_{J_{u}} \varphi\left(u^{+}, u^{-}, v_{u}\right) \mathrm{d} \mathcal{H}^{1} .
$$

This concludes the proof.

To conclude the proof of the main theorems, it remains to show Proposition 2.2, Theorem 2.5, and Proposition 3.4. This is subject to the next sections. 


\section{Cell Formula Part I: Relation of $L^{1}$-convergence and Boundary Values}

In this first part about cell formulas, we show that the condition of $L^{1}$-convergence as given in the cell formula $\psi$, see (3.5), can be replaced by converging boundary values. More precisely, in this section we consider $\Phi: \mathcal{Z} \times \mathcal{Z} \times \mathbb{S}^{1} \rightarrow[0,+\infty)$ defined by

$$
\begin{aligned}
\Phi\left(z^{+}, z^{-}, v\right)=\min & \left\{\operatorname { l i m i n f } _ { \varepsilon \rightarrow 0 } \operatorname { i n f } \left\{E_{\varepsilon}\left(X_{\varepsilon}, Q^{v}\left(y_{\varepsilon}\right)\right): y_{\varepsilon} \in \mathbb{R}^{2}, X_{\varepsilon}\right.\right. \\
& \left.\left.=\varepsilon \mathscr{L}\left(z_{\varepsilon}^{ \pm}\right) \text {on } \partial_{\varepsilon}^{ \pm} Q^{v}\left(y_{\varepsilon}\right)\right\}:\left\{z_{\varepsilon}^{ \pm}\right\}_{\varepsilon} \subset \mathcal{Z} \text { with } z_{\varepsilon}^{ \pm} \rightarrow z^{ \pm}\right\},
\end{aligned}
$$

where the identity $X_{\varepsilon}=\varepsilon \mathscr{L}\left(z_{\varepsilon}^{ \pm}\right)$is defined in (2.10) and $\partial_{\varepsilon}^{ \pm} Q^{v}\left(y_{\varepsilon}\right)$ in (2.7). This means that near the boundary of the cube the configuration is contained in at most two different lattices $\varepsilon \mathscr{L}\left(z_{\varepsilon}^{ \pm}\right)$. (Less is possible if $z_{\varepsilon}^{ \pm}=\mathbf{0}$.) We note that the minimum in (4.1) is attained by a standard diagonal sequence argument. Our aim is to prove the following statement:

Lemma 4.1. (Relation of $\psi$ and $\Phi)$ Let $z^{+}, z^{-} \in \mathcal{Z}$ and $v \in \mathbb{S}^{1}$. Then

$$
\psi\left(z^{+}, z^{-}, v\right) \geqq \Phi\left(z^{+}, z^{-}, v\right) .
$$

In Section 7, we will prove $\Phi\left(z^{+}, z^{-}, v\right)=\varphi\left(z^{+}, z^{-}, v\right)$ for all $z^{+}, z^{-} \in \mathcal{Z}$ and $v \in \mathbb{S}^{1}$, see Lemma 7.1, and Proposition 7.2. This along with Lemma 4.1 will conclude the proof of Proposition 3.4.

As it is customary in the analysis of cell formulas, the proof of Lemma 4.1 crucially relies on a cut-off argument which allows to construct configurations attaining the boundary values. Whereas for problems on Sobolev spaces this is usually achieved by a convex combination of functions, our discrete problem is considerably more delicate. In fact, on the one hand, the system is quite flexible due to the rotational and translational invariance of the atomistic energy, cf. Lemma 3.1(i). On the other hand, the system is very rigid as small changes in the configuration may induce a lot of energy due to the discontinuous interaction potential, see (1.1). This calls for a refined cut-off construction.

The construction fundamentally relies on the fact that the energy of an optimal sequence in (3.5) is concentrated asymptotically arbitrarily close to the interface. (Similar properties can be observed in related phase transition problems, see for example $[12,13,15]$.) As a preliminary step, we need to show that in the definition of $\psi$ we may replace cubes by rectangles. To this end, we introduce half-open rectangles with sides parallel to $v$ by

$$
R_{l, h}^{v}(y)=y+\left\{x \in \mathbb{R}^{2}:-\frac{h}{2} \leqq\langle x, v\rangle<\frac{h}{2},-\frac{l}{2} \leqq\left\langle x, v^{\perp}\right\rangle<\frac{l}{2}\right\},
$$

where $y \in \mathbb{R}^{2}$, and $l, h>0$. We simply write $R_{l, h}^{v}$ instead of $R_{l, h}^{v}(y)$ if the rectangle is centered at $y=0$. Recall the definition in (3.4). 
Lemma 4.2. (Density $\psi$ on rectangles) For all $z^{+}, z^{-} \in \mathcal{Z}$, all $v \in \mathbb{S}^{1}$, and all $l, h>0$ there holds

$$
\begin{aligned}
\psi\left(z^{+}, z^{-}, v\right)=\inf & \left\{\liminf _{\varepsilon \rightarrow 0} \frac{1}{l} E_{\varepsilon}\left(X_{\varepsilon}, R_{l, h}^{v}\left(y_{\varepsilon}\right)\right): y_{\varepsilon} \in \mathbb{R}^{2},\right. \\
& \left.\lim _{\varepsilon \rightarrow 0} \int_{R_{l, h}^{v}}\left|u_{\varepsilon}\left(x+y_{\varepsilon}\right)-u_{z^{+}, z^{-}}^{v}(x)\right| \mathrm{d} x=0\right\} .
\end{aligned}
$$

Proof. For convenience, we denote the function on the right hand side of (4.4) in the variables $\left(z^{+}, z^{-}, v, l, h\right)$ by $\Psi$. We will use certain scaling properties of $\Psi$ :

$$
\begin{aligned}
& \Psi\left(z^{+}, z^{-}, v, \lambda \ell, \lambda \kappa\right)=\Psi\left(z^{+}, z^{-}, v, \ell, \kappa\right) \text { for all } \lambda>0 \\
& \Psi\left(z^{+}, z^{-}, v, \ell, \kappa\right) \leqq \Psi\left(z^{+}, z^{-}, v, \ell, \lambda \kappa\right) \text { for all } \lambda \geqq 1 \\
& \Psi\left(z^{+}, z^{-}, v, \ell, \kappa\right) \leqq \Psi\left(z^{+}, z^{-}, v, \lambda \ell, \kappa\right) \text { for all } \lambda \in \mathbb{N} \\
& \ell_{1} \Psi\left(z^{+}, z^{-}, v, \ell_{1}, \kappa\right) \leqq \ell_{2} \Psi\left(z^{+}, z^{-}, v, \ell_{2}, \kappa\right) \text { for all } 0<\ell_{1} \leqq \ell_{2} .
\end{aligned}
$$

We postpone the proof of (4.5)-(4.8) to Step 3 of the proof, and first derive the statement.

Step 1: Independence of $l$. We start by proving the independence of the length $l$, that is,

$$
\Psi\left(z^{+}, z^{-}, v, l, h\right)=\Psi\left(z^{+}, z^{-}, v, \mu l, h\right)
$$

for all $\mu>0$. To this end, consider first $\mu \in \mathbb{N}$. Using (4.5) and then (4.6) with $\lambda=\mu, \ell=l$, and $\kappa=h / \mu$, we obtain

$$
\Psi\left(z^{+}, z^{-}, v, \mu l, h\right)=\Psi\left(z^{+}, z^{-}, v, l, h / \mu\right) \leqq \Psi\left(z^{+}, z^{-}, v, l, h\right) .
$$

By (4.7) for $\mu \in \mathbb{N}$ it holds that $\Psi\left(z^{+}, z^{-}, v, \mu l, h\right) \geqq \Psi\left(z^{+}, z^{-}, v, l, h\right)$. Combining the estimates we get

$$
\Psi\left(z^{+}, z^{-}, v, \mu l, h\right)=\Psi\left(z^{+}, z^{-}, v, l, h\right)
$$

for $\mu \in \mathbb{N}$. Now substituting $l$ with $\frac{l}{\mu}$ in the previous equation, we obtain

$$
\Psi\left(z^{+}, z^{-}, v, l, h\right)=\Psi\left(z^{+}, z^{-}, v, l / \mu, h\right)
$$

for all $\mu \in \mathbb{N}$ and $l>0$. Hence, due to (4.10) and (4.11), equality (4.9) holds for all $\mu \in \mathbb{Q}^{+}$.

Now, for general $\mu>0$, we take a sequence $\left\{\mu_{n}\right\}_{n} \subset \mathbb{Q}$ such that $\mu_{n} \leqq \mu_{n+1}$ for all $n \in \mathbb{N}$ and $\mu_{n} \rightarrow \mu$. By (4.8) and the fact that (4.9) holds for all $\mu \in \mathbb{Q}$, we obtain

$$
\Psi\left(z^{+}, z^{-}, v, l, h\right)=\Psi\left(z^{+}, z^{-}, v, \mu_{n} l, h\right) \leqq \frac{\mu}{\mu_{n}} \Psi\left(z^{+}, z^{-}, v, \mu l, h\right) .
$$

Taking $n \rightarrow+\infty$, we obtain

$$
\Psi\left(z^{+}, z^{-}, v, l, h\right) \leqq \Psi\left(z^{+}, z^{-}, v, \mu l, h\right) .
$$


This yields one inequality in (4.9). Applying (4.12) for $\lambda$ in place of $\mu$ and $l / \lambda$ in place of $l$ we also get

$$
\Psi\left(z^{+}, z^{-}, v, l, h\right)=\Psi\left(z^{+}, z^{-}, v, \lambda l / \lambda, h\right) \geqq \Psi\left(z^{+}, z^{-}, v, l / \lambda, h\right) .
$$

If we choose $\lambda=\mu^{-1}$, we get the other inequality in (4.9).

Step 2: Independence of $h$. Let $\mu>0$. By first applying (4.5) and then (4.9) we obtain

$$
\Psi\left(z^{+}, z^{-}, v, l, h\right)=\Psi\left(z^{+}, z^{-}, v, \mu l, \mu h\right)=\Psi\left(z^{+}, z^{-}, v, l, \mu h\right) .
$$

This yields the desired independence of the height $h$.

Step 3: Proof of (4.5)-(4.8). It remains to prove (4.5)-(4.8).

Step 3.1: Proof of (4.5). Fix $\lambda, \ell, \kappa>0$. Let $X_{\varepsilon} \subset \mathbb{R}^{2}$ and $y_{\varepsilon} \in \mathbb{R}^{2}$ be given such that $\lim _{\varepsilon \rightarrow 0} \int_{R_{\ell, \kappa}^{v}}\left|u_{\varepsilon}\left(x+y_{\varepsilon}\right)-u_{z^{+}, z^{-}}^{v}(x)\right| \mathrm{d} x=0$ and

$$
\Psi\left(z^{+}, z^{-}, v, \ell, \kappa\right)=\liminf _{\varepsilon \rightarrow 0} \frac{1}{\ell} E_{\varepsilon}\left(X_{\varepsilon}, R_{\ell, \kappa}^{v}\left(y_{\varepsilon}\right)\right) .
$$

(By a standard diagonal sequence argument the infimum on the right hand side of (4.4) is attained.) Set $X_{\varepsilon}^{\lambda}=\lambda X_{\varepsilon}$. By (3.1) we get that the corresponding functions $u_{\lambda \varepsilon}^{\lambda}$, see (2.15), satisfy $u_{\lambda \varepsilon}^{\lambda}(x)=u_{\varepsilon}\left(\lambda^{-1} x\right)$ for all $x \in \mathbb{R}^{2}$. Change of variables $y=\lambda^{-1} x$ and $u_{z^{+}, z^{-}}^{v}(y)=u_{z^{+}, z^{-}}^{v}(\lambda y)$ imply

$$
\begin{aligned}
& \lim _{\varepsilon \rightarrow 0} \int_{R_{\lambda \ell, \lambda \kappa}^{v}}\left|u_{\lambda \varepsilon}^{\lambda}\left(x+\lambda y_{\varepsilon}\right)-u_{z^{+}, z^{-}}^{\nu}(x)\right| \mathrm{d} x \\
& \quad=\lim _{\varepsilon \rightarrow 0} \lambda^{2} \int_{R_{\ell, \kappa}^{v}}\left|u_{\varepsilon}\left(y+y_{\varepsilon}\right)-u_{z^{+}, z^{-}}^{v}(y)\right| \mathrm{d} y=0 .
\end{aligned}
$$

Using Lemma 3.1(ii) along with (4.13) and the definition of $\Psi$, we obtain

$$
\begin{aligned}
\Psi\left(z^{+}, z^{-}, v, \lambda \ell, \lambda \kappa\right) & \leqq \liminf _{\varepsilon \rightarrow 0} \frac{1}{\lambda \ell} E_{\lambda \varepsilon}\left(X_{\varepsilon}^{\lambda}, R_{\lambda \ell, \lambda \kappa}^{\nu}\left(\lambda y_{\varepsilon}\right)\right) \\
& =\liminf _{\varepsilon \rightarrow 0} \frac{1}{\ell} E_{\varepsilon}\left(X_{\varepsilon}, R_{\ell, \kappa}^{\nu}\left(y_{\varepsilon}\right)\right)=\Psi\left(z^{+}, z^{-}, \nu, \ell, \kappa\right) .
\end{aligned}
$$

By exchanging $\lambda$ with $\frac{1}{\lambda}$ and $\ell, \kappa$ with $\lambda \ell, \lambda \kappa$, respectively, we obtain (4.5).

Step 3.2: Proof of (4.6). Fix $\lambda \geqq 1$ and $\ell, \kappa>0$. Consider $X_{\varepsilon} \subset \mathbb{R}^{2}$ and $y_{\varepsilon} \in \mathbb{R}^{2}$ such that $\lim _{\varepsilon \rightarrow 0} \int_{R_{\ell, \lambda \kappa}^{v}}\left|u_{\varepsilon}\left(x+y_{\varepsilon}\right)-u_{z^{+}, z^{-}}^{v}(x)\right| \mathrm{d} x=0$ and

$$
\Psi\left(z^{+}, z^{-}, \nu, \ell, \lambda \kappa\right)=\liminf _{\varepsilon \rightarrow 0} \frac{1}{\ell} E_{\varepsilon}\left(X_{\varepsilon}, R_{\ell, \lambda \kappa}^{\nu}\left(y_{\varepsilon}\right)\right) .
$$

By Lemma 3.1(iii) and the definition of $\Psi$ we get

$$
\begin{aligned}
& \Psi\left(z^{+}, z^{-}, v, \ell, \kappa\right) \leqq \liminf _{\varepsilon \rightarrow 0} \frac{1}{\ell} E_{\varepsilon}\left(X_{\varepsilon}, R_{\ell, \kappa}^{v}\left(y_{\varepsilon}\right)\right) \leqq \liminf _{\varepsilon \rightarrow 0} \frac{1}{\ell} E_{\varepsilon}\left(X_{\varepsilon}, R_{\ell, \lambda \kappa}^{v}\left(y_{\varepsilon}\right)\right) \\
& \quad=\Psi\left(z^{+}, z^{-}, v, \ell, \lambda \kappa\right) .
\end{aligned}
$$


Step 3.3: Proof of (4.7). Let $\lambda \in \mathbb{N}$ and $\ell, \kappa>0$. Consider $X_{\varepsilon} \subset \mathbb{R}^{2}$ and $y_{\varepsilon} \in \mathbb{R}^{2}$ such that

$$
\lim _{\varepsilon \rightarrow 0} \int_{R_{\lambda \ell, \kappa}^{v}}\left|u_{\varepsilon}\left(x+y_{\varepsilon}\right)-u_{z^{+}, z^{-}}^{v}(x)\right| \mathrm{d} x=0
$$

and

$$
\Psi\left(z^{+}, z^{-}, v, \lambda \ell, \kappa\right)=\liminf _{\varepsilon \rightarrow 0} \frac{1}{\lambda \ell} E_{\varepsilon}\left(X_{\varepsilon}, R_{\lambda \ell, \kappa}^{v}\left(y_{\varepsilon}\right)\right) .
$$

We decompose the half-open rectangle $R_{\lambda \ell, \kappa}^{v}\left(y_{\varepsilon}\right)$ into pairwise disjoint half-open rectangles of the form

$$
R_{\lambda \ell, \kappa}^{v}\left(y_{\varepsilon}\right)=\bigcup_{j=0}^{\lambda-1} R_{\ell, \kappa}^{v}\left(y_{j}^{\varepsilon}\right)
$$

where $y_{j}^{\varepsilon}=y_{\varepsilon}+\frac{2 j-\lambda+1}{2} \ell \nu^{\perp}$. Now, using Lemma 3.1(iv), we derive that there exists $j_{0}$ such that

$$
E_{\varepsilon}\left(X_{\varepsilon}, R_{\ell, \kappa}^{\nu}\left(y_{j_{0}}^{\varepsilon}\right)\right) \leqq \frac{1}{\lambda} \sum_{j=0}^{\lambda-1} E_{\varepsilon}\left(X_{\varepsilon}, R_{\ell, \kappa}^{\nu}\left(y_{j}^{\varepsilon}\right)\right)=\frac{1}{\lambda} E_{\varepsilon}\left(X_{\varepsilon}, R_{\lambda \ell, \kappa}^{\nu}\left(y_{\varepsilon}\right)\right) .
$$

By (4.14) and the fact that $u_{z^{+}, z^{-}}^{v}(x)=u_{z^{+}, z^{-}}^{v}\left(x+t v^{\perp}\right)$ for all $x \in \mathbb{R}^{2}$ and $t \in \mathbb{R}$, see (3.4), we get that $\lim _{\varepsilon \rightarrow 0} \int_{R_{\ell, \kappa}^{v}}\left|u_{\varepsilon}\left(x+y_{j_{0}}^{\varepsilon}\right)-u_{z^{+}, z^{-}}^{v}(x)\right| \mathrm{d} x=0$. By the definition of $\Psi,(4.15)$, and (4.16) this yields

$$
\begin{aligned}
& \Psi\left(z^{+}, z^{-}, v, \ell, \kappa\right) \leqq \liminf _{\varepsilon \rightarrow 0} \frac{1}{\ell} E_{\varepsilon}\left(X_{\varepsilon}, R_{\ell, \kappa}^{v}\left(y_{j_{0}}^{\varepsilon}\right)\right) \leqq \liminf _{\varepsilon \rightarrow 0} \frac{1}{\lambda \ell} E_{\varepsilon}\left(X_{\varepsilon}, R_{\lambda \ell, \kappa}^{v}\left(y_{\varepsilon}\right)\right) \\
& \quad=\Psi\left(z^{+}, z^{-}, v, \lambda \ell, \kappa\right) .
\end{aligned}
$$

This implies (4.7).

Step 3.4: Proof of (4.8). Let $0<\ell_{1} \leqq \ell_{2}$. Consider $X_{\varepsilon} \subset \mathbb{R}^{2}$ and $y_{\varepsilon} \in \mathbb{R}^{2}$ such that $\lim _{\varepsilon \rightarrow 0} \int_{R_{\ell_{2}, \kappa}^{v}}\left|u_{\varepsilon}\left(x+y_{\varepsilon}\right)-u_{z^{+}, z^{-}}^{v}(x)\right| \mathrm{d} x=0$ and

$$
\Psi\left(z^{+}, z^{-}, v, \ell_{2}, \kappa\right)=\liminf _{\varepsilon \rightarrow 0} \frac{1}{\ell_{2}} E_{\varepsilon}\left(X_{\varepsilon}, R_{\ell_{2}, \kappa}^{v}\left(y_{\varepsilon}\right)\right) .
$$

By using Lemma 3.1(iii) along with $\ell_{2} \geqq \ell_{1}$ and the definition of $\Psi$ we get

$$
\begin{aligned}
\Psi\left(z^{+}, z^{-}, v, \ell_{1}, \kappa\right) & \leqq \liminf _{\varepsilon \rightarrow 0} \frac{1}{\ell_{1}} E_{\varepsilon}\left(X_{\varepsilon}, R_{\ell_{1}, \kappa}^{v}\left(y_{\varepsilon}\right)\right) \leqq \liminf _{\varepsilon \rightarrow 0} \frac{1}{\ell_{1}} E_{\varepsilon}\left(X_{\varepsilon}, R_{\ell_{2}, \kappa}^{v}\left(y_{\varepsilon}\right)\right) \\
& =\frac{\ell_{2}}{\ell_{1}} \liminf _{\varepsilon \rightarrow 0} \frac{1}{\ell_{2}} E_{\varepsilon}\left(X_{\varepsilon}, R_{\ell_{2}, \kappa}^{v}\left(y_{\varepsilon}\right)\right)=\frac{\ell_{2}}{\ell_{1}} \Psi\left(z^{+}, z^{-}, v, \ell_{2}, \kappa\right) .
\end{aligned}
$$

This yields (4.8) and concludes the proof.

We now proceed with the proof of Lemma 4.1. 
Proof of Lemma 4.1. In view of (3.5), we can choose a subsequence in $\varepsilon$ (not relabeled) and configurations $X_{\varepsilon} \subset \mathbb{R}^{2}$ and $y_{\varepsilon} \in \mathbb{R}^{2}$ such that $\lim _{\varepsilon \rightarrow 0} \int_{Q^{v}} \mid u_{\varepsilon}(x+$ $\left.y_{\varepsilon}\right)-u_{z^{+}, z^{-}}^{v}(x) \mid \mathrm{d} x=0$ and

$$
\psi\left(z^{+}, z^{-}, v\right)=\lim _{\varepsilon \rightarrow 0} E_{\varepsilon}\left(X_{\varepsilon}, Q^{v}\left(y_{\varepsilon}\right)\right) .
$$

We perform a refined cut-off construction and split the proof into several steps. As explained above, the construction is quite delicate due to the fact that the energy is very sensitive to small changes of the configurations. First, we use Lemma 4.2 to prove that the energy of $X_{\varepsilon}$ concentrates around a strip close to the limiting interface (Step 1). This allows us to select one dominant component on each side of the interface, that is, on the upper and the lower half-cube (Step 2). Here, the notion "component" refers to a subset of a specific triangular lattice.

Our goal in the subsequent steps is to modify the configuration $X_{\varepsilon}$ such that it coincides with these lattices near the boundary of the upper and lower half-cube, respectively. In Step 3, we give a precise cardinality estimate on the number of points that differ from the lattices of the two dominant components in terms of $\mathrm{o}\left(\varepsilon^{-2}\right)$. In Step 4, we select a "good layer" where we can modify our configuration. "Good" means here that, in that layer, the configuration coincides with the lattice of the dominant component up to $o\left(\varepsilon^{-1}\right)$ atoms. In Step 5, we show that the configuration constructed in Step 4 is an asymptotic energy lower bound for the original configuration. Finally, in Step 6, we conclude by observing that the constructed configuration is a competitor in the definition of $\Phi$. We will perform this construction under the assumption that in both the upper and the lower half-cube there exist (dominant) lattices. The case of vacuum calls for small adaptions which are described at the end in Step 7.

Step 1: The energy concentrates near the line $\left\{\left\langle v,\left(x-y_{\varepsilon}\right)\right\rangle=0\right\}$. Recall (4.3). We show that for all $\delta \in(0,1)$ it holds that

$$
\lim _{\varepsilon \rightarrow 0} E_{\varepsilon}\left(X_{\varepsilon}, Q^{v}\left(y_{\varepsilon}\right) \backslash R_{1, \delta}^{v}\left(y_{\varepsilon}\right)\right)=0 .
$$

By Lemma 3.1(iii), Lemma 4.2, (4.17), and the fact that $\left\{X_{\varepsilon}\right\}_{\varepsilon}$ is admissible in the definition of $\psi$ on $R_{1, \delta}^{v}$, see (4.4), we obtain

$\psi\left(z^{+}, z^{-}, v\right) \leqq \liminf _{\varepsilon \rightarrow 0} E_{\varepsilon}\left(X_{\varepsilon}, R_{1, \delta}^{v}\left(y_{\varepsilon}\right)\right) \leqq \lim _{\varepsilon \rightarrow 0} E_{\varepsilon}\left(X_{\varepsilon}, Q^{v}\left(y_{\varepsilon}\right)\right)=\psi\left(z^{+}, z^{-}, v\right)$.

Lemma 3.1(iv) then implies

$$
\begin{aligned}
0 & \leqq \limsup _{\varepsilon \rightarrow 0} E_{\varepsilon}\left(X_{\varepsilon}, Q^{v}\left(y_{\varepsilon}\right) \backslash R_{1, \delta}^{v}\left(y_{\varepsilon}\right)\right) \\
& =\limsup _{\varepsilon \rightarrow 0}\left(E_{\varepsilon}\left(X_{\varepsilon}, Q^{v}\left(y_{\varepsilon}\right)\right)-E_{\varepsilon}\left(X_{\varepsilon}, R_{1, \delta}^{v}\left(y_{\varepsilon}\right)\right)\right) \\
& \leqq \lim _{\varepsilon \rightarrow 0} E_{\varepsilon}\left(X_{\varepsilon}, Q^{v}\left(y_{\varepsilon}\right)\right)-\liminf _{\varepsilon \rightarrow 0} E_{\varepsilon}\left(X_{\varepsilon}, R_{1, \delta}^{v}\left(y_{\varepsilon}\right)\right)=0 .
\end{aligned}
$$

This yields (4.18) and concludes Step 1.

In order to shorten the notation, we omit the dependence on the center $y_{\varepsilon}$ and simply write $Q_{\rho}^{v}$ instead of $Q_{\rho}^{v}\left(y_{\varepsilon}\right)$ for $\rho>0$ and $R_{1, \delta}^{v}$ instead of $R_{1, \delta}^{v}\left(y_{\varepsilon}\right)$. For 
brevity, we also define (omitting the center $y_{\varepsilon}$ ) the rectangles $P_{\delta, \varepsilon}^{ \pm}=Q_{1-\varepsilon}^{\nu, \pm} \backslash R_{1-\varepsilon, \delta}^{v}$, where $Q_{1-\varepsilon}^{\nu, \pm}$ is defined below (2.6). We will prove all auxiliary statements along the proof for the upper half-cube $Q^{\nu,+}$ only since the arguments for the lower one are analogous. In what follows, $\delta \in(0,1)$ is fixed sufficiently small. Without restriction, we may suppose that $\varepsilon \ll \delta$.

Step 2: Single dominant component in the upper and lower half. We prove that there exist sequences $\left\{z_{\varepsilon}^{ \pm}\right\}_{\varepsilon} \subset \mathcal{Z}$ such that $z_{\varepsilon}^{ \pm} \rightarrow z^{ \pm}$and

$$
\mathcal{L}^{2}\left(\left\{u_{\varepsilon} \neq z_{\varepsilon}^{ \pm}\right\} \cap P_{\delta, \varepsilon}^{ \pm}\right) \leqq C E_{\varepsilon}\left(X_{\varepsilon}, Q^{v} \backslash R_{1, \delta / 2}^{v}\right)
$$

where $C>0$ is a universal constant independent of $\varepsilon$.

Recall by (2.11) and (2.15) that the function $u_{\varepsilon}$ can be written as $u_{\varepsilon}=$ $\sum_{j=1}^{\infty} \chi_{G_{j}^{\varepsilon}} z_{j}^{\varepsilon}$ for pairwise distinct $\left\{z_{j}^{\varepsilon}\right\}_{j} \subset \mathcal{Z} \backslash\{\mathbf{0}\}$ and pairwise disjoint $\left\{G_{j}^{\varepsilon}\right\}_{j} \subset$ $\mathbb{R}^{2}$. By Proposition 3.3 (more precisely, see (3.3)), (2.4), and Lemma 3.1(iii) we have

$$
\sum_{j=1}^{\infty} \mathcal{H}^{1}\left(\partial^{*} G_{j}^{\varepsilon} \cap P_{\delta, \varepsilon}^{+}\right) \leqq C E_{\varepsilon}\left(X_{\varepsilon},\left(P_{\delta, \varepsilon}^{+}\right)_{\varepsilon}\right) \leqq C E_{\varepsilon}\left(X_{\varepsilon}, Q^{v} \backslash R_{1, \delta / 2}^{v}\right),
$$

where in the last step we used $\left(P_{\delta, \varepsilon}^{+}\right)_{\varepsilon} \subset Q^{\nu} \backslash R_{1, \delta / 2}^{v}$. We also define the vacuum inside $Q^{\nu}$ by $G_{0}^{\varepsilon}:=Q^{\nu} \backslash \bigcup_{j=1}^{\infty} G_{j}^{\varepsilon}$. By the relative isoperimetric inequality (see for example [22, Theorem 2, Section 5.6.2]), there exists $c>0$ such that for all $j \in \mathbb{N}_{0}$ that holds

$$
\begin{aligned}
\min \left\{\mathcal{L}^{2}\left(G_{j}^{\varepsilon} \cap P_{\delta, \varepsilon}^{+}\right)\right. & \left., \mathcal{L}^{2}\left(P_{\delta, \varepsilon}^{+} \backslash G_{j}^{\varepsilon}\right)\right\} \\
& \leqq \min \left\{\mathcal{L}^{2}\left(G_{j}^{\varepsilon} \cap P_{\delta, \varepsilon}^{+}\right), \mathcal{L}^{2}\left(P_{\delta, \varepsilon}^{+} \backslash G_{j}^{\varepsilon}\right)\right\}^{1 / 2} \mathcal{L}^{2}\left(P_{\delta, \varepsilon}^{+}\right)^{1 / 2} \\
& \leqq c \mathcal{H}^{1}\left(\partial^{*} G_{j}^{\varepsilon} \cap P_{\delta, \varepsilon}^{+}\right)
\end{aligned}
$$

where we used $\mathcal{L}^{2}\left(P_{\delta, \varepsilon}^{+}\right) \leqq 1$. (Note that the theorem in the reference above is stated and proved in a ball, but that the argument only relies on Poincaré inequalities, and thus easily extends to the rectangles $P_{\delta, \varepsilon}^{+}$. Since the ratio of length and width is controlled, the constant is independent of $\delta$ and $\varepsilon$.) Then, from (4.20), (4.21), and $\partial^{*} G_{0}^{\varepsilon} \cap P_{\delta, \varepsilon}^{+} \subset \bigcup_{j=1}^{\infty}\left(\partial^{*} G_{j}^{\varepsilon} \cap P_{\delta, \varepsilon}^{+}\right)$it follows that

$$
\sum_{j=0}^{\infty} \min \left\{\mathcal{L}^{2}\left(G_{j}^{\varepsilon} \cap P_{\delta, \varepsilon}^{+}\right), \mathcal{L}^{2}\left(P_{\delta, \varepsilon}^{+} \backslash G_{j}^{\varepsilon}\right)\right\} \leqq C E_{\varepsilon}\left(X_{\varepsilon}, Q^{v} \backslash R_{1, \delta / 2}^{v}\right) .
$$

We now get that there is a unique dominant component, that is, there exists $j_{\varepsilon} \in \mathbb{N}_{0}$ such that

$$
\mathcal{L}^{2}\left(G_{j_{\varepsilon}}^{\varepsilon} \cap P_{\delta, \varepsilon}^{+}\right)>\frac{1}{2} \mathcal{L}^{2}\left(P_{\delta, \varepsilon}^{+}\right)
$$

In fact, assume by contradiction that this were not the case. Then, we get, for all $j \in \mathbb{N}_{0}$,

$$
\min \left\{\mathcal{L}^{2}\left(G_{j}^{\varepsilon} \cap P_{\delta, \varepsilon}^{+}\right), \mathcal{L}^{2}\left(P_{\delta, \varepsilon}^{+} \backslash G_{j}^{\varepsilon}\right)\right\}=\mathcal{L}^{2}\left(G_{j}^{\varepsilon} \cap P_{\delta, \varepsilon}^{+}\right)
$$


By using (4.22) we obtain $\mathcal{L}^{2}\left(P_{\delta, \varepsilon}^{+}\right)=\sum_{j=0}^{\infty} \mathcal{L}^{2}\left(G_{j}^{\varepsilon} \cap P_{\delta, \varepsilon}^{+}\right) \leqq C E_{\varepsilon}\left(X_{\varepsilon}, Q^{v} \backslash R_{1, \delta / 2}^{v}\right)$. This contradicts (4.18) for $\varepsilon$ small enough. Now (4.22) and (4.23) imply (4.19) for the choice $z_{\varepsilon}^{+}=z_{j_{\varepsilon}}^{\varepsilon}$.

To conclude this step, we note that the convergence $\lim _{\varepsilon \rightarrow 0} \int_{Q^{v}} \mid u_{\varepsilon}\left(x+y_{\varepsilon}\right)-$ $u_{z^{+}, z^{-}}^{v}(x) \mid \mathrm{d} x=0$ along with (4.23) also yields $z_{\varepsilon}^{+} \rightarrow z^{+}$.

The rest of the proof is divided into two cases: (a) $z_{\varepsilon}^{+} \neq \mathbf{0}$ and (b) $z_{\varepsilon}^{+}=\mathbf{0}$, that is, $X_{\varepsilon}$ converges to a lattice in the upper half of the cube or there is vacuum. We perform the proof for case (a). At the end of the proof (Step 7), we indicate the necessary changes to treat case (b).

Step 3: Cardinality estimate. We prove that there exists $C>0$ such that

$$
\varepsilon^{2} \#\left(\left(\varepsilon \mathscr{L}\left(z_{\varepsilon}^{ \pm}\right) \triangle X_{\varepsilon}\right) \cap P_{\delta, \varepsilon}^{ \pm}\right) \leqq C E_{\varepsilon}\left(X_{\varepsilon}, Q^{v} \backslash R_{1, \delta / 2}^{v}\right),
$$

where here and in what follows $\Delta$ denotes the symmetric difference of sets. First, consider some $x \in\left(\varepsilon \mathscr{L}\left(z_{\varepsilon}^{+}\right) \backslash X_{\varepsilon}\right) \cap P_{\delta, \varepsilon}^{+}$. Then, by the definition of $u_{\varepsilon}$ in (2.15) we get

$$
u_{\varepsilon}(y) \neq z_{\varepsilon}^{+} \text {for all } y \in B_{\varepsilon / 4}(x)
$$

Indeed, otherwise we would find $y \in B_{\varepsilon / 4}(x)$ and $x^{\prime} \in X_{\varepsilon} \cap B_{\varepsilon / \sqrt{3}}(y)$ with $\# \mathcal{N}_{\varepsilon}\left(x^{\prime}\right)=6$ and $\left\{x^{\prime}\right\} \cup \mathcal{N}_{\varepsilon}\left(x^{\prime}\right) \subset \varepsilon \mathscr{L}\left(z_{\varepsilon}^{+}\right)$. The latter follows from the fact that $V_{\varepsilon}^{z_{\varepsilon}^{+}}\left(x^{\prime}\right) \subset B_{\varepsilon / \sqrt{3}}\left(x^{\prime}\right)$. In particular, we have $x^{\prime} \in \varepsilon \mathscr{L}\left(z_{\varepsilon}^{+}\right)$and $\left|x-x^{\prime}\right| \leqq$ $|x-y|+\left|y-x^{\prime}\right| \leqq \varepsilon / 4+\varepsilon / \sqrt{3}<\varepsilon$. This, however, is impossible, since $\left|x_{1}-x_{2}\right| \geqq \varepsilon$ for all $x_{1}, x_{2} \in \varepsilon \mathscr{L}\left(z_{\varepsilon}^{+}\right), x_{1} \neq x_{2}$.

On the other hand, if there exists $x \in\left(X_{\varepsilon} \backslash \varepsilon \mathscr{L}\left(z_{\varepsilon}^{+}\right)\right) \cap P_{\delta, \varepsilon}^{+}$, then we find $x_{0} \in \varepsilon \mathscr{L}\left(z_{\varepsilon}^{+}\right) \cap P_{\delta, \varepsilon}^{+}$with $\left|x_{0}-x\right|<\varepsilon$. Clearly, $x_{0} \notin X_{\varepsilon}$ by (1.1) and the fact that $E_{\varepsilon}\left(X_{\varepsilon}\right)<+\infty$. Repeating the reasoning in (4.25) we find that

$$
u_{\varepsilon}(y) \neq z_{\varepsilon}^{+} \text {for all } y \in B_{\varepsilon / 4}\left(x_{0}\right) .
$$

Note that, in this procedure, $x_{0}$ can be chosen for at most six $x \in X_{\varepsilon}$ independently of $\varepsilon$ since $\#\left(X_{\varepsilon} \cap B_{\varepsilon}\left(x_{0}\right)\right) \leqq 6$ due to $E_{\varepsilon}\left(X_{\varepsilon}\right)<+\infty$. Using (4.19), $\mathcal{L}^{2}\left(B_{\varepsilon / 4}(x) \cap P_{\delta, \varepsilon}^{+}\right) \geqq c \varepsilon^{2}$ for all $x \in \varepsilon \mathscr{L}\left(z_{\varepsilon}^{+}\right) \cap P_{\delta, \varepsilon}^{+}$, and (4.25)-(4.26) we conclude $\varepsilon^{2} \#\left(\left(\varepsilon \mathscr{L}\left(z_{\varepsilon}^{+}\right) \triangle X_{\varepsilon}\right) \cap P_{\delta, \varepsilon}^{+}\right) \leqq C \mathcal{L}^{2}\left(\left\{u_{\varepsilon} \neq z_{\varepsilon}^{+}\right\} \cap P_{\delta, \varepsilon}^{+}\right) \leqq C E_{\varepsilon}\left(X_{\varepsilon}, Q^{\nu} \backslash R_{1, \delta / 2}^{v}\right)$.

Step 4: Cut-off construction. In this step, we construct a new configuration $Y_{\varepsilon}^{+} \subset \mathbb{R}^{2}$ such that $Y_{\varepsilon}^{+}=\varepsilon \mathscr{L}\left(z_{\varepsilon}^{+}\right)$on $\partial_{\varepsilon}^{+} Q^{v}$, see (2.7). This construction changes the configuration in the upper half-cube $Q^{\nu,+}$. Step 5 then shows that the energy of $Y_{\varepsilon}^{+}$is asymptotically equal to the one of $X_{\varepsilon}$. The procedure can then be repeated on the lower half-cube. We defer this to Step 6 below.

Set $N_{\varepsilon}=\left\lfloor\frac{\delta}{6 \varepsilon}\right\rfloor$. (Here and in the sequel, we do not highlight the dependence on $\delta$ to save notation.) For $k \in\left\{0, \ldots, N_{\varepsilon}+1\right\}$ we let $r_{k}=1-\delta+3 k \varepsilon$ and define the layers

$$
S_{k}^{\varepsilon}=\left(Q_{r_{k}}^{\nu,+} \backslash Q_{r_{k-1}}^{\nu,+}\right) \backslash R_{1, \delta}^{v} .
$$


For $k \in\left\{1, \ldots, N_{\varepsilon}\right\}$ we also define the "thickened layers" $L_{k}^{\varepsilon}=S_{k-1}^{\varepsilon} \cup S_{k}^{\varepsilon} \cup S_{k+1}^{\varepsilon}$. Our goal is to perform a transition to the lattice $\varepsilon \mathscr{L}\left(z_{\varepsilon}^{+}\right)$on one of these layers. To this end, we choose a convenient layer by an averaging argument: by (4.24) there exists $k_{\varepsilon} \in\left\{1, \ldots, N_{\varepsilon}\right\}$ such that

$$
\begin{aligned}
\#\left(\left(\varepsilon \mathscr{L}\left(z_{\varepsilon}^{+}\right) \triangle X_{\varepsilon}\right) \cap L_{k_{\varepsilon}}^{\varepsilon}\right) & \leqq \frac{1}{N_{\varepsilon}} \sum_{k=1}^{N_{\varepsilon}} \#\left(\left(\varepsilon \mathscr{L}\left(z_{\varepsilon}^{+}\right) \triangle X_{\varepsilon}\right) \cap L_{k}^{\varepsilon}\right) \\
& \leqq \frac{3}{N_{\varepsilon}} \#\left(\left(\varepsilon \mathscr{L}\left(z_{\varepsilon}^{+}\right) \triangle X_{\varepsilon}\right) \cap P_{\delta, \varepsilon}^{+}\right) \leqq \frac{C}{\varepsilon \delta} E_{\varepsilon}\left(X_{\varepsilon}, Q^{v} \backslash R_{1, \delta / 2}^{v}\right) .
\end{aligned}
$$

Here, we used $L_{k}^{\varepsilon} \subset P_{\delta, \varepsilon}^{+}$for all $k$ and $\varepsilon \delta \leqq C N_{\varepsilon} \varepsilon^{2}$. The factor 3 is due to the fact that we count each strip $S_{k}^{\varepsilon}$ at most three times. Set $D^{\varepsilon}:=Q_{r_{k_{\varepsilon}-1}}^{v} \cup\left(Q^{\nu,-} \backslash R_{1, \delta}^{v}\right)$. We now define $Y_{\varepsilon}^{+}$by

$$
Y_{\varepsilon}^{+}= \begin{cases}\varepsilon \mathscr{L}\left(z_{\varepsilon}^{+}\right) & \text {in }\left(P_{\delta, \varepsilon}^{+} \backslash Q_{r_{\varepsilon}}^{v}\right) \cup \partial_{\varepsilon}^{+} Q^{v}, \\ \varnothing & \text { in }\left(R_{1, \delta}^{v} \backslash Q_{r_{k_{\varepsilon}-1}}^{v}\right) \backslash\left(\partial_{\varepsilon}^{+} Q^{v} \cup \partial_{\varepsilon}^{-} Q^{v}\right), \\ X_{\varepsilon} \cap \varepsilon \mathscr{L}\left(z_{\varepsilon}^{+}\right) & \text {in } S_{k_{\varepsilon}}^{\varepsilon}, \\ X_{\varepsilon} & \text { in } D^{\varepsilon} \cup \partial_{\varepsilon}^{-} Q^{v} .\end{cases}
$$

See Fig. 7 for an illustration of the different regions. We briefly explain the definition. In $D^{\varepsilon} \cup \partial_{\varepsilon}^{-} Q^{\nu}$, the configuration remains unchanged, and near the boundary of the upper half-cube it coincides with the lattice $\varepsilon \mathscr{L}\left(z_{\varepsilon}^{+}\right)$. In $S_{k_{\varepsilon}}^{\varepsilon}$, we use the intersection $X_{\varepsilon} \cap \varepsilon \mathscr{L}\left(z_{\varepsilon}^{+}\right)$. In this sense, $S_{k_{\varepsilon}}^{\varepsilon}$ can be understood as a transition layer. Eventually, small regions near the boundary close to the interface $\partial Q^{\nu,+} \cap \partial Q^{\nu,-}$ do not contain atoms. This is convenient since in this region the energy of the original configuration possibly does not vanish. Note that the latter ensures that $\left|y_{1}-y_{2}\right| \geqq \varepsilon$ for all $y_{1}, y_{2} \in Y_{\varepsilon}^{+}, y_{1} \neq y_{2}$, and therefore

$$
E_{\varepsilon}\left(Y_{\varepsilon}^{+}\right)<+\infty \text {. }
$$

Finally, we point out that $Y_{\varepsilon}^{+} \not \subset Q^{v}$ due to the definition of $\partial_{\varepsilon}^{ \pm} Q^{v}$ in (2.7), see also Fig. 3.

Step 5: Energy estimate. In this step we show that the energy of the configuration constructed in Step 4 is asymptotically controlled by the original energy, that is,

$$
\liminf _{\varepsilon \rightarrow 0} E_{\varepsilon}\left(Y_{\varepsilon}^{+}, Q^{v}\right) \leqq \liminf _{\varepsilon \rightarrow 0} E_{\varepsilon}\left(X_{\varepsilon}, Q^{v}\right)+C \delta
$$

for some universal $C>0$. In order to obtain (4.31), we distinguish three regions:

$$
A_{1}^{\varepsilon}=\overline{\left(R_{1, \delta}^{v} \backslash Q_{r_{k-1}}^{v}\right)_{\varepsilon}}, \quad A_{2}^{\varepsilon}=\overline{\left(S_{k_{\varepsilon}}^{\varepsilon}\right)_{\varepsilon}} \backslash A_{1}^{\varepsilon}, \quad A_{3}^{\varepsilon}=Q^{v} \backslash\left(A_{1}^{\varepsilon} \cup A_{2}^{\varepsilon}\right) .
$$

Energy estimate on $A_{1}^{\varepsilon}$ : We claim that there exists a universal $C>0$ such that

$$
E_{\varepsilon}\left(Y_{\varepsilon}^{+}, A_{1}^{\varepsilon}\right) \leqq C \delta
$$

In fact, due to (4.29), we have $Y_{\varepsilon}^{+} \cap\left(R_{1, \delta}^{v} \backslash Q_{r_{k_{\varepsilon}-1}}^{v}\right)=\left(\varepsilon \mathscr{L}\left(z_{\varepsilon}^{+}\right) \cap R_{1, \delta}^{v} \cap \partial_{\varepsilon}^{+} Q^{v}\right) \cup$ $\left(X_{\varepsilon} \cap R_{1, \delta}^{v} \cap \partial_{\varepsilon}^{-} Q^{v}\right)$. As $\mathcal{L}^{2}\left(\left(R_{1, \delta}^{v} \cap \partial_{\varepsilon}^{ \pm} Q^{v}\right)_{\varepsilon}\right) \leqq C \delta \varepsilon$, see (2.7) and (4.3), by Lemma 3.1(v) we get

$$
\#\left(Y_{\varepsilon}^{+} \cap\left(R_{1, \delta}^{v} \backslash Q_{r_{\varepsilon}-1}^{v}\right)\right) \leqq C \delta / \varepsilon .
$$




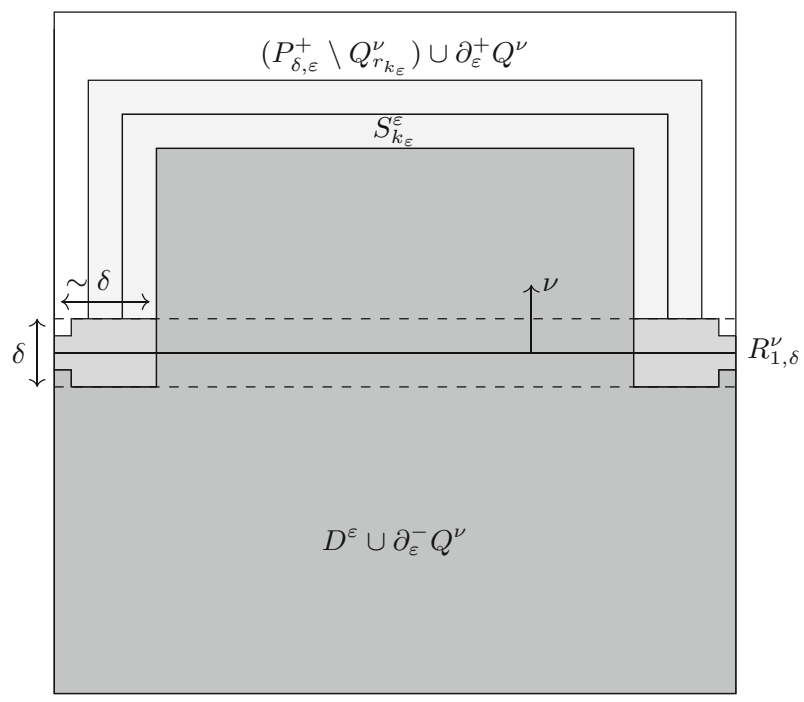

Fig. 7. The different regions for $Y_{\varepsilon}^{+}$inside $Q^{\nu}$ : dark gray region $D^{\varepsilon} \cup \partial_{\varepsilon}^{-} Q^{\nu}$, gray region $\left(R_{1, \delta}^{v} \backslash Q_{r_{k_{\varepsilon}-1}}^{v}\right) \backslash\left(\partial_{\varepsilon}^{+} Q^{\nu} \cup \partial_{\varepsilon}^{-} Q^{\nu}\right)$, light gray region $S_{k_{\varepsilon}}^{\varepsilon}$, and white region $\left(P_{\delta, \varepsilon}^{+} \backslash Q_{r_{k_{\varepsilon}}}^{v}\right) \cup$ $\partial_{\varepsilon}^{+} Q^{\nu}$. The two dashed lines enclose the region $R_{1, \delta}^{\nu}$

Here, Lemma 3.1 is applicable by (4.30). Additionally, we note that $R_{1, \delta}^{v} \backslash Q_{r_{k_{\varepsilon}-1}}^{v}$ consists of two rectangles and we have $\mathcal{H}^{1}\left(\partial\left(R_{1, \delta}^{v} \backslash Q_{r_{k_{\varepsilon}-1}}^{v}\right)\right) \leqq C \delta$. Hence, by Lemma 3.1(v) we obtain

$$
\begin{aligned}
\#\left(\left(A_{1}^{\varepsilon} \cap Y_{\varepsilon}^{+}\right) \backslash\left(R_{1, \delta}^{v} \backslash Q_{r_{k_{\varepsilon}-1}}^{v}\right)\right) & \left.\leqq C \varepsilon^{-2} \mathcal{L}^{2}\left(\overline{\left(R_{1, \delta}^{v} \backslash Q_{r_{k_{\varepsilon}-1}}^{v}\right)_{\varepsilon}} \backslash\left(R_{1, \delta}^{v} \backslash Q_{r_{k_{\varepsilon}-1}}^{v}\right)\right)_{\varepsilon}\right) \\
& \leqq C \varepsilon^{-1} \mathcal{H}^{1}\left(\partial\left(R_{1, \delta}^{v} \backslash Q_{r_{k_{\varepsilon}-1}}^{v}\right)\right) \leqq C \delta / \varepsilon .
\end{aligned}
$$

This along with (4.34) yields \# $\left(A_{1}^{\varepsilon} \cap Y_{\varepsilon}^{+}\right) \leqq C \delta / \varepsilon$, and therefore (4.33) follows by (2.3).

Energy estimate on $A_{2}^{\varepsilon}$ : We prove that there exists a universal $C>0$ such that

$$
E_{\varepsilon}\left(Y_{\varepsilon}^{+}, A_{2}^{\varepsilon}\right) \leqq(1+C / \delta) E_{\varepsilon}\left(X_{\varepsilon}, Q^{v} \backslash R_{1, \delta / 2}^{v}\right) .
$$

First, the definition of $L_{k_{\varepsilon}}^{\varepsilon}$ below (4.27) implies $\left(A_{2}^{\varepsilon}\right)_{\varepsilon} \subset L_{k_{\varepsilon}}^{\varepsilon}$. For $x \in Y_{\varepsilon}^{+}$, we denote the neighborhood of $x$ with respect to $Y_{\varepsilon}^{+}$by $\mathcal{N}_{\varepsilon, Y}(x)$, cf. (2.1). We claim that $\# \mathcal{N}_{\varepsilon, Y}(x) \geqq \# \mathcal{N}_{\varepsilon}(x)-6 \#\left(\overline{B_{\varepsilon}(x)} \cap\left(X_{\varepsilon} \backslash \varepsilon \mathscr{L}\left(z_{\varepsilon}^{+}\right)\right)\right) \quad$ for all $x \in X_{\varepsilon} \cap Y_{\varepsilon}^{+} \cap A_{2}^{\varepsilon}$.

In fact, if $\overline{B_{\varepsilon}(x)} \cap\left(X_{\varepsilon} \backslash \varepsilon \mathscr{L}\left(z_{\varepsilon}^{+}\right)\right) \neq \varnothing$, the right hand side is nonpositive since $\# \mathcal{N}_{\varepsilon}(x) \leqq 6$, see (2.2). Since $\# \mathcal{N}_{\varepsilon, Y}(x) \geqq 0$, (4.36) follows in this case. On the other hand, if $\overline{B_{\varepsilon}(x)} \cap\left(X_{\varepsilon} \backslash \varepsilon \mathscr{L}\left(z_{\varepsilon}^{+}\right)\right)=\emptyset$, by (4.29), we may have only increased the cardinality of the neighborhood by adding atoms in $\varepsilon \mathscr{L}\left(z_{\varepsilon}^{+}\right) \backslash X_{\varepsilon}$, that is, $\# \mathcal{N}_{\varepsilon, Y}(x) \geqq \# \mathcal{N}_{\varepsilon}(x)$. This again yields (4.36). 
We split the sum into $X_{\varepsilon} \cap Y_{\varepsilon}^{+}$and $Y_{\varepsilon}^{+} \backslash X_{\varepsilon}$. By using (2.3), $A_{2}^{\varepsilon} \subset L_{k_{\varepsilon}}^{\varepsilon}$, Lemma 3.1(iii), and (4.36) we obtain

$$
\begin{aligned}
& E_{\varepsilon}\left(Y_{\varepsilon}^{+}, A_{2}^{\varepsilon}\right) \leqq C \varepsilon \#\left\{x \in A_{2}^{\varepsilon} \cap\left(Y_{\varepsilon}^{+} \backslash X_{\varepsilon}\right)\right\}+\frac{1}{2} \sum_{\substack{x \in Y_{\varepsilon}^{+} \cap X_{\varepsilon} \\
x \in A_{2}^{\varepsilon}}} \varepsilon\left(6-\# \mathcal{N}_{\varepsilon, Y}(x)\right) \\
& \leqq C \varepsilon \#\left\{x \in\left(Y_{\varepsilon}^{+} \cap L_{k_{\varepsilon}}^{\varepsilon}\right) \backslash X_{\varepsilon}\right\}+3 \varepsilon \sum_{\substack{x \in Y_{\varepsilon}^{+} \cap X_{\varepsilon} \\
x \in A_{2}^{\varepsilon}}} \#\left(\overline{B_{\varepsilon}(x)} \cap\left(X_{\varepsilon} \backslash \varepsilon \mathscr{L}\left(z_{\varepsilon}^{+}\right)\right)\right) \\
& \quad+E_{\varepsilon}\left(X_{\varepsilon}, L_{k_{\varepsilon}}^{\varepsilon}\right) .
\end{aligned}
$$

Note, by (4.29), that $Y_{\varepsilon}^{+} \subset \varepsilon \mathscr{L}\left(z_{\varepsilon}^{+}\right) \cup X_{\varepsilon}$ in $L_{k_{\varepsilon}}^{\varepsilon}$. Therefore, in view of (4.28), we obtain

$\#\left\{x \in\left(Y_{\varepsilon}^{+} \cap L_{k_{\varepsilon}}^{\varepsilon}\right) \backslash X_{\varepsilon}\right\} \leqq \#\left\{x \in\left(\varepsilon \mathscr{L}\left(z_{\varepsilon}^{+}\right) \triangle X_{\varepsilon}\right) \cap L_{k_{\varepsilon}}^{\varepsilon}\right\} \leqq \frac{C}{\varepsilon \delta} E_{\varepsilon}\left(X_{\varepsilon}, Q^{v} \backslash R_{1, \delta / 2}^{v}\right)$.

Exploiting (4.28) once more, we get

$$
\begin{aligned}
\sum_{x \in Y_{\varepsilon}^{+} \cap X_{\varepsilon} \cap A_{2}^{\varepsilon}} \#\left(\overline{B_{\varepsilon}(x)} \cap\left(X_{\varepsilon} \backslash \varepsilon \mathscr{L}\left(z_{\varepsilon}^{+}\right)\right)\right) & \leqq C \#\left\{x \in\left(\varepsilon \mathscr{L}\left(z_{\varepsilon}^{+}\right) \triangle X_{\varepsilon}\right) \cap L_{k_{\varepsilon}}^{\varepsilon}\right\} \\
& \leqq \frac{C}{\varepsilon \delta} E_{\varepsilon}\left(X_{\varepsilon}, Q^{v} \backslash R_{1, \delta / 2}^{v}\right) .
\end{aligned}
$$

Here, the first inequality holds because $\left|x_{1}-x_{2}\right| \geqq \varepsilon$ for $x_{1}, x_{2} \in X_{\varepsilon}, x_{1} \neq x_{2}$, and $\overline{B_{\varepsilon}(x)} \subset L_{k_{\varepsilon}}^{\varepsilon}$ for all $x \in A_{2}^{\varepsilon}$. Hence, we get that every point in $\left(X_{\varepsilon} \backslash \varepsilon \mathcal{L}\left(z_{\varepsilon}^{+}\right)\right) \cap L_{k_{\varepsilon}}^{\varepsilon}$ is only accounted for at most seven times in the sum. Now, using (4.37)-(4.39), $L_{k_{\varepsilon}}^{\varepsilon} \subset Q^{v} \backslash R_{1, \delta}^{v}$, and Lemma 3.1(iii), we obtain (4.35).

Energy estimate on $A_{3}^{\varepsilon}$ : We claim that

$$
E_{\varepsilon}\left(Y_{\varepsilon}^{+}, A_{3}^{\varepsilon}\right) \leqq E_{\varepsilon}\left(X_{\varepsilon}, Q^{v}\right) .
$$

Recalling (4.32) we get that each $x \in A_{3}^{\varepsilon} \cap Y_{\varepsilon}^{+}$lies either in $T^{\varepsilon}:=\left(P_{\delta, \varepsilon}^{+} \backslash Q_{r_{\varepsilon}}^{v}\right) \cup$ $\left(\partial_{\varepsilon}^{+} Q^{v} \backslash R_{1, \delta}^{v}\right.$ ) or in $D^{\varepsilon}$. If $x \in A_{3}^{\varepsilon} \cap Y_{\varepsilon}^{+} \cap T^{\varepsilon}$, then also $\overline{B_{\varepsilon}(x)} \subset T^{\varepsilon}$. (Here, we use the definition of $A_{1}^{\varepsilon}, A_{2}^{\varepsilon}$ and (2.7).) Then, (4.29) implies \# $\mathcal{N}_{\varepsilon, Y}(x)=6$. On the other hand, if $x \in A_{3}^{\varepsilon} \cap Y_{\varepsilon}^{+} \cap D^{\varepsilon}$, then $X_{\varepsilon} \cap \overline{B_{\varepsilon}(x)}=Y_{\varepsilon}^{+} \cap \overline{B_{\varepsilon}(x)}$, which yields $\mathcal{N}_{\varepsilon, Y}(x)=$ $\mathcal{N}_{\varepsilon}(x)$. Thus, by (2.3) and Lemma 3.1(iii),(iv) we obtain (4.40). In fact, we get

$$
\begin{aligned}
E_{\varepsilon}\left(Y_{\varepsilon}^{+}, A_{3}^{\varepsilon}\right) & =E_{\varepsilon}\left(Y_{\varepsilon}^{+}, A_{3}^{\varepsilon} \cap T^{\varepsilon}\right)+E_{\varepsilon}\left(Y_{\varepsilon}^{+}, A_{3}^{\varepsilon} \cap D^{\varepsilon}\right) \\
& =E_{\varepsilon}\left(Y_{\varepsilon}^{+}, A_{3}^{\varepsilon} \cap D^{\varepsilon}\right) \leqq E_{\varepsilon}\left(X_{\varepsilon}, Q^{\nu}\right) .
\end{aligned}
$$

To conclude this step of the proof, it suffices to recall that by Lemma 3.1(iv)

$$
E_{\varepsilon}\left(Y_{\varepsilon}^{+}, Q^{\nu}\right)=E_{\varepsilon}\left(Y_{\varepsilon}^{+}, A_{1}^{\varepsilon}\right)+E_{\varepsilon}\left(Y_{\varepsilon}^{+}, A_{2}^{\varepsilon}\right)+E_{\varepsilon}\left(Y_{\varepsilon}^{+}, A_{3}^{\varepsilon}\right) .
$$

Then we obtain (4.31) by (4.18), (4.33), (4.35), and (4.40). 
Step 6: Conclusion. By repeating the cut-off construction in Step 4 on $Q^{v,-}$ for $z_{\varepsilon}^{-}$, we obtain a configuration $Y_{\varepsilon}$ such that $Y_{\varepsilon}=\varepsilon \mathscr{L}\left(z_{\varepsilon}^{ \pm}\right)$on $\partial_{\varepsilon}^{ \pm} Q^{\nu}\left(y_{\varepsilon}\right)$ and

$$
\liminf _{\varepsilon \rightarrow 0} E_{\varepsilon}\left(Y_{\varepsilon}, Q^{\nu}\left(y_{\varepsilon}\right)\right) \leqq \liminf _{\varepsilon \rightarrow 0} E_{\varepsilon}\left(X_{\varepsilon}, Q^{\nu}\left(y_{\varepsilon}\right)\right)+C \delta
$$

by (4.31), where we reinclude the center $y_{\varepsilon}$ in the notation for clarification. Since $z_{\varepsilon}^{ \pm} \rightarrow z^{ \pm}$by Step 2, we observe by the definition of $\Phi$ in (4.1) that

$$
\liminf _{\varepsilon \rightarrow 0} E_{\varepsilon}\left(Y_{\varepsilon}, Q^{v}\left(y_{\varepsilon}\right)\right) \geqq \Phi\left(z^{+}, z^{-}, v\right) .
$$

By using (4.17), (4.41) and by passing to $\delta \rightarrow 0$, we obtain the statement of the lemma.

Step 7: Adaptions in (b). To conclude the proof of the lemma, it remains to describe Steps 3-5 in the case of vacuum, that is, $z_{\varepsilon}^{+}=\mathbf{0}$.

Step 3 for case (b): Cardinality estimate. We prove that

$$
\varepsilon^{2} \#\left(X_{\varepsilon} \cap P_{\delta, \varepsilon}^{+}\right) \leqq C E_{\varepsilon}\left(X_{\varepsilon}, Q^{v} \backslash R_{1, \delta / 2}^{v}\right)
$$

for a universal $C>0$. In fact, if $x \in X_{\varepsilon}$ has $\# \mathcal{N}_{\varepsilon}(x)=6$, then $u_{\varepsilon}(x) \neq \mathbf{0}$ on $B_{\varepsilon / 2}(x)$ by (2.15) and the fact that $B_{\varepsilon / 2}(x) \subset V_{\varepsilon}^{z(x)}(x)$. Also note the $B_{\varepsilon / 2}(x) \cap B_{\varepsilon / 2}(y)=\varnothing$ for $x, y \in X_{\varepsilon}, x \neq y$. Thus, by (2.3), (4.19) (with $z_{\varepsilon}^{+}=\mathbf{0}$ ), and Lemma 3.1(iii) we get

$$
\begin{gathered}
\varepsilon^{2} \#\left(X_{\varepsilon} \cap P_{\delta, \varepsilon}^{+}\right) \leqq \varepsilon^{2} \#\left\{x \in X_{\varepsilon} \cap P_{\delta, \varepsilon}^{+}: \# \mathcal{N}_{\varepsilon}(x)=6\right\}+\varepsilon^{2} \sum_{x \in X_{\varepsilon} \cap P_{\delta, \varepsilon}^{+}}\left(6-\# \mathcal{N}_{\varepsilon}(x)\right) \\
\leqq C \mathcal{L}^{2}\left(\left\{u_{\varepsilon} \neq \mathbf{0}\right\} \cap P_{\delta, \varepsilon}^{+}\right)+2 \varepsilon E_{\varepsilon}\left(X_{\varepsilon}, Q^{\nu} \backslash R_{1, \delta / 2}^{v}\right) \leqq C E_{\varepsilon}\left(X_{\varepsilon}, Q^{v} \backslash R_{1, \delta / 2}^{v}\right),
\end{gathered}
$$

where we again used that $P_{\delta, \varepsilon}^{+} \subset Q^{v} \backslash R_{1, \delta / 2}^{v}$. This concludes Step 3 in case (b). Step 4 for case (b): Cut-off construction. We now explain the construction of a new configuration $Y_{\varepsilon}^{+}$such that $Y_{\varepsilon}^{+}=\mathbf{0}$ on $\partial_{\varepsilon}^{+} Q^{v}$. Again set $N_{\varepsilon}=\left\lfloor\frac{\delta}{6 \varepsilon}\right\rfloor$ and define $S_{k}^{\varepsilon}$ as in (4.27), as well as $L_{k}^{\varepsilon}=S_{k-1}^{\varepsilon} \cup S_{k}^{\varepsilon} \cup S_{k+1}^{\varepsilon}$. Similar to (4.28), by averaging over $k$ and using (4.42), there exists $k_{\varepsilon} \in\left\{1, \ldots, N_{\varepsilon}\right\}$ such that

$$
\#\left(X_{\varepsilon} \cap L_{k_{\varepsilon}}^{\varepsilon}\right) \leqq \frac{1}{N_{\varepsilon}} \sum_{k=1}^{N_{\varepsilon}} \#\left(X_{\varepsilon} \cap L_{k}^{\varepsilon}\right) \leqq \frac{3}{N_{\varepsilon}} \#\left(X_{\varepsilon} \cap P_{\delta, \varepsilon}^{+}\right) \leqq \frac{C}{\varepsilon \delta} E_{\varepsilon}\left(X_{\varepsilon}, Q^{v} \backslash R_{1, \delta / 2}^{v}\right),
$$

where we again use that each strip $S_{k}^{\varepsilon}$ is counted at most three times. We define

$$
Y_{\varepsilon}^{+}= \begin{cases}\emptyset & \text { in }\left(\left(P_{\delta, \varepsilon}^{+} \cup R_{1, \delta}^{v}\right) \backslash\left(Q_{r_{k_{\varepsilon}}}^{v} \cup \partial_{\varepsilon}^{-} Q^{v}\right)\right) \cup \partial_{\varepsilon}^{+} Q^{v}, \\ X_{\varepsilon} & \text { otherwise. }\end{cases}
$$

Note that, since $E_{\varepsilon}\left(X_{\varepsilon}\right)<+\infty$, we have that $E_{\varepsilon}\left(Y_{\varepsilon}^{+}\right)<+\infty$.

Step 5 for case (b): Energy estimate. We again split the estimate into the three sets $A_{1}^{\varepsilon}, A_{2}^{\varepsilon}$, and $A_{3}^{\varepsilon}$ defined in (4.32).

Energy estimate for $A_{1}^{\varepsilon}$ : We claim that there exists $C>0$ such that

$$
E_{\varepsilon}\left(Y_{\varepsilon}^{+}, A_{1}^{\varepsilon}\right) \leqq C \delta
$$


In fact, due to (4.44), we have $Y_{\varepsilon}^{+} \cap\left(R_{1, \delta}^{v} \backslash Q_{r_{k_{\varepsilon}}}^{v}\right)=X_{\varepsilon} \cap R_{1, \delta}^{v} \cap \partial_{\varepsilon}^{-} Q^{v}$, where, similarly as in (4.34), \# $\left(X_{\varepsilon} \cap R_{1, \delta}^{v} \cap \partial_{\varepsilon}^{-} Q^{v}\right) \leqq C \delta / \varepsilon$. As $R_{1, \delta}^{v} \backslash Q_{r_{k_{\varepsilon}-1}}^{v}$ consists of two rectangles with $\mathcal{H}^{1}\left(\partial\left(R_{1, \delta}^{v} \backslash Q_{r_{k_{\varepsilon}}-1}^{v}\right)\right) \leqq C \delta$ and $Y_{\varepsilon}^{+}$satisfies $E_{\varepsilon}\left(Y_{\varepsilon}^{+}\right)<+\infty$, we obtain, by Lemma 3.1(v)

$$
\begin{aligned}
\#\left(A_{1}^{\varepsilon} \cap Y_{\varepsilon}^{+}\right) & =\#\left(\left(A_{1}^{\varepsilon} \backslash\left(R_{1, \delta}^{v} \backslash Q_{r_{k_{\varepsilon}}}^{v}\right)\right) \cap Y_{\varepsilon}^{+}\right)+\#\left(X_{\varepsilon} \cap R_{1, \delta}^{v} \cap \partial_{\varepsilon}^{-} Q^{v}\right) \\
& \leqq C \varepsilon^{-2} \mathcal{L}^{2}\left(\left(A_{1}^{\varepsilon} \backslash\left(R_{1, \delta}^{v} \backslash Q_{r_{k_{\varepsilon}}}^{v}\right)\right)_{\varepsilon}\right)+C \delta / \varepsilon \\
& \leqq C \varepsilon^{-1} \mathcal{H}^{1}\left(\partial\left(R_{1, \delta}^{v} \backslash Q_{r_{k_{\varepsilon}-1}}^{v}\right)\right)+C \delta / \varepsilon \leqq C \delta / \varepsilon
\end{aligned}
$$

Then (4.45) follows by (2.3).

Energy estimate for $A_{2}^{\varepsilon}$ : We claim that there exists $C>0$ such that

$$
E_{\varepsilon}\left(Y_{\varepsilon}^{+}, A_{2}^{\varepsilon}\right) \leqq \frac{C}{\delta} E_{\varepsilon}\left(X_{\varepsilon}, Q^{v} \backslash R_{1, \delta / 2}^{v}\right)
$$

In fact, if $x \in Y_{\varepsilon}^{+} \cap A_{2}^{\varepsilon}$, then $x \in X_{\varepsilon} \cap L_{k_{\varepsilon}}^{\varepsilon}$. Using (2.3) and (4.43) we obtain (4.46). Energy estimate for $A_{3}^{\varepsilon}$ : We observe that

$$
E_{\varepsilon}\left(Y_{\varepsilon}^{+}, A_{3}^{\varepsilon}\right) \leqq E_{\varepsilon}\left(X_{\varepsilon}, Q^{v}\right) .
$$

Indeed, if $x \in Y_{\varepsilon}^{+} \cap\left(Q^{v} \backslash\left(A_{1}^{\varepsilon} \cup A_{2}^{\varepsilon}\right)\right)$, then $\mathcal{N}_{\varepsilon, Y}(x)=\mathcal{N}_{\varepsilon}(x)$, where the neighborhood of $x$ with respect to $Y_{\varepsilon}^{+}$is again denoted by $\mathcal{N}_{\varepsilon, Y}(x)$. Therefore, (4.47) follows by (2.3) and Lemma 3.1(iii).

Summarizing, (4.45)-(4.47) and (4.18) yield

$$
\liminf _{\varepsilon \rightarrow 0} E_{\varepsilon}\left(Y_{\varepsilon}^{+}, Q^{v}\right) \leqq \liminf _{\varepsilon \rightarrow 0} E_{\varepsilon}\left(X_{\varepsilon}, Q^{v}\right)+C \delta,
$$

which is the analog to (4.31). The rest of the proof (that is, Step 6) remains unchanged.

\section{Reduction of the Problem to Subsets of Two Lattices}

In the previous section, we have seen that the condition of $L^{1}$-convergence in the definition of $\psi$ (see (3.5)) can be replaced by converging boundary values, see the definition of $\Phi$ in (4.1). From now on, it will be convenient to express the problem with lattice spacing equal to 1 . Recall (2.7) and observe that by Lemma 3.1 the cell formula for $\Phi$ can be written as

$$
\begin{aligned}
\Phi\left(z^{+}, z^{-}, v\right)=\min & \left\{\operatorname { l i m i n f } _ { T \rightarrow + \infty } \frac { 1 } { T } \operatorname { i n f } \left\{E_{1}\left(X_{T}, Q_{T}^{v}\left(y_{T}\right)\right): y_{T} \in \mathbb{R}^{2},\right.\right. \\
& \left.\left.X_{T}=\mathscr{L}\left(z_{T}^{ \pm}\right) \text {on } \partial_{1}^{ \pm} Q_{T}^{v}\left(y_{T}\right)\right\}:\left\{z_{T}^{ \pm}\right\}_{T} \subset \mathcal{Z} \text { with } z_{T}^{ \pm} \rightarrow z^{ \pm}\right\}
\end{aligned}
$$

for all $z^{+}, z^{-} \in \mathcal{Z}$ and $v \in \mathbb{S}^{1}$. This section is devoted to a fundamental ingredient for the proof of relation of $\Phi$ and $\varphi$, and the properties of $\varphi$, which will be addressed in Sections 6 and 7. We show that the minimization problem in (5.1) can be reduced 
to configurations that are subsets of two lattices only (or just one if either $z^{+}=\mathbf{0}$ or $z^{-}=\mathbf{0}$ ). For the formulation of the lemma, we introduce two further notions: we say that a set $Y \subset \mathbb{R}^{2}$ is connected if for each pair $x, y \in Y$ there exists a chain $\left(v_{1}, \ldots, v_{n}\right)$ with $v_{i} \in Y$ for $i \in\{1, \ldots, n\}, v_{1}=x, v_{n}=y$, and $\left|v_{i+1}-v_{i}\right|=1$ for $i \in\{1, \ldots, n-1\}$. Moreover, given a configuration $X$ and $Y \subset X$, we define the boundary of $Y$ inside $Q_{T}^{v}(y)$ by

$$
\partial Y=\left\{x \in Y \cap Q_{T}^{v}(y): \#(\mathcal{N}(x) \cap Y)<6\right\} .
$$

Lemma 5.1. (Reduction to subsets of two lattices) Let $z^{+}, z^{-} \in \mathcal{Z}, v \in \mathbb{S}^{1}, y \in \mathbb{R}^{2}$, and $T>0$. Let $X \subset \mathbb{R}^{2}$ be a minimizer of

$$
\min \left\{E_{1}\left(X, Q_{T}^{v}(y)\right): X=\mathscr{L}\left(z^{ \pm}\right) \text {on } \partial_{1}^{ \pm} Q_{T}^{v}(y)\right\} .
$$

Then, it satisfies the following two properties:

(i) (Subset of lattices) There holds $X=X^{+} \cup X^{-}$on $Q_{T}^{v}(y)$, where $X^{ \pm} \subset \mathscr{L}\left(z^{ \pm}\right)$ and $X^{ \pm}$is connected.

(ii) (Structure of boundaries) The sets $\partial X^{+}$and $\partial X^{-}$defined in (5.2) are connected and satisfy $\# \mathcal{N}(x) \leqq 5$ for all $x \in \partial X^{ \pm}$, as well as $\max _{x, y \in \partial X^{ \pm}}|x-y| \geqq T$.

Note that the minimum in (5.3) exists since $E_{1}$ is lower semicontinuous, see (1.1) and (1.3), and the problem is finite dimensional. We also point out that $X^{+} \cap X^{-} \neq \varnothing$ is possible, see for example Fig. 4, that is, the two grains described by $X^{+}$and $X^{-}$ can have common atoms. Resolving this ambiguity by introducing a specific choice, the grain boundary and bonds connecting the two grains can be described in more detail.

Lemma 5.2. (Bonds between grain boundaries) Let $X^{ \pm}$be the sets found in Lemma 5.1. There exist $Y^{ \pm}$with $X^{ \pm} \backslash \partial X^{\mp} \subset Y^{ \pm} \subset X^{ \pm}$such that

(i) (Partition into grains) $Y^{+} \cup Y^{-}=X^{+} \cup X^{-}$and $Y^{+} \cap Y^{-} \cap Q_{T}^{v}(y)=\emptyset$.

(ii) (Grain and bulk boundaries) $\partial Y^{ \pm} \subset \partial X^{ \pm}$and $Y^{ \pm}=\mathscr{L}\left(z^{ \pm}\right)$on $\partial_{1}^{ \pm} Q_{T}^{v}(y)$.

(iii) (Neighborhood structure at grain boundary) it holds that

$$
\left|\sum_{x \in \partial Y^{ \pm}} \#\left(\mathcal{N}(x) \cap Y^{ \pm}\right)-4 \# \partial Y^{ \pm}\right| \leqq 2 .
$$

We thus have that on average each boundary atom has four neighbors in the same grain. As it has at most five neighbors in the whole configuration, it has on average less than one bond connecting it to the other grain.

From a technical perspective, Lemma 5.1 will provide an important tool to study the properties of the cell formulas. From the physical point of view, it shows that our extremely brittle set-up, while allowing for rebonding, does not support interpolating boundary layers near cracks. Its proof will require some concepts from graph theory which will be only needed for this part of the article. For this reason, it is possible to omit the proofs of Lemmas 5.1 and 5.2 on first reading and to proceed directly with Section 6. As our graph theoretic description gives in fact a more precise picture of the geometry of grain boundaries, which is of some 
independent interest, we summarize these findings in Theorem 5.4 at the end of Section 5.

We now address the proof of the lemma and start by introducing some notions from graph theory.

The bond graph: We define the bond graph of $X \subset \mathbb{R}^{2}$ as the set of positions $X$ with the set of bonds $\{\{x, y\}: x \in X, y \in \mathcal{N}(x)\}$, where $\mathcal{N}(x)=\mathcal{N}_{1}(x)$ is defined in (2.1). As for configurations with finite energy $E_{1}$ there holds $\operatorname{dist}(x, X \backslash\{x\}) \geqq 1$ for all $x \in X$ and $y \in \mathcal{N}(x)$ only if $|x-y|=1<\sqrt{2}$, the bond graph is planar. Indeed, given a quadrilateral with all sides and one diagonal equal to 1 , the second diagonal is $\sqrt{3}>1$.

A sequence of atoms $p=\left(v_{1}, \ldots, v_{n}\right) \subset X$ is called a simple path in $X$ if the atoms are distinct and $\left\{v_{j-1}, v_{j}\right\}$ are bonds for $j \in\{1, \ldots, n-1\}$. If $\left(v_{1}, \ldots, v_{n-1}\right)$ is a simple path and $v_{n-1}$ is connected to $v_{n}=v_{1}$ by a bond, $p$ is a cycle in $X$. We say that a configuration is connected if each two atoms are joinable through a simple path. (Note that this definition is consistent with the one given before the statement of Lemma 5.1.) A bond is called acyclic if it is not contained in any cycle of the bond graph. The reduced bond graph of $X$ is obtained by first deleting all acyclic bonds and then all atoms which are not connected to any other atom. By a face of $X$ we always mean a face of its reduced bond graph. The boundary of a face is given by a disjoint union of cycles and by a unique cycle if the reduced bond graph is connected. Such a boundary is called a polygon and, in particular, a $j$-gon if it consists of $j \in \mathbb{N}$ atoms.

Sub-configuration: We say that $Z \subset X$ is a sub-configuration of $X$. All notions defined above are defined analogously for any sub-configuration $Z$ of $X$.

Face defect: We define the face defect of a sub-configuration $Z \subset X$ by

$$
\eta(Z)=\sum_{j \geqq 3}(j-3) f_{j}(Z),
$$

where $f_{j}(Z)$ denotes the number of polygons with $j$ atoms in the bond graph of $Z$. Strong connectedness: We say that a configuration $Z$ is strongly connected if $Z \backslash\{x\}$ is connected for every $x \in Z$. Note that strongly connected graphs with more than two atoms coincide with their reduced bond graph as they do not contain acyclic bonds since removing one of the atoms belonging to the bond would disconnect the configuration.

Maximal components: Fix $Q_{T}^{v}(y)$. Let $z^{+}, z^{-} \in \mathcal{Z}$ and consider $X \subset \mathbb{R}^{2}$ such that $X=\mathscr{L}\left(z^{ \pm}\right)$on $\partial_{1}^{ \pm} Q_{T}^{v}(y)$. We denote the set of strongly connected subsets of lattices by

$$
\mathcal{C}^{ \pm}=\left\{Z \subset X \cap \mathscr{L}\left(z^{ \pm}\right): Z \cap \partial_{1}^{ \pm} Q_{T}^{v}(y) \neq \emptyset, Z \text { is strongly connected }\right\} .
$$

We introduce the maximal components, denoted by $M^{ \pm}$, as the maximal elements in $\mathcal{C}^{ \pm}$with respect to set inclusion. These sets can be written as

$$
M^{ \pm}=\bigcup_{Z \in \mathcal{C}^{ \pm}} Z
$$




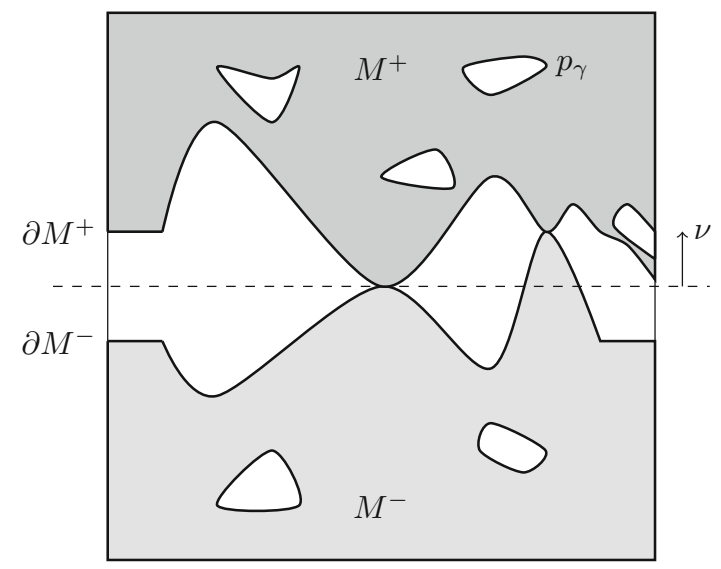

Fig. 8. A schematic picture of $M^{+} \cap Q_{T}^{v}(y)$, depicted in dark gray, and of $M^{-} \cap Q_{T}^{v}(y)$, depicted in light gray. Their boundaries are illustrated in bold. We depict also a curve $p_{\gamma}$ considered in Step 2 of the proof below
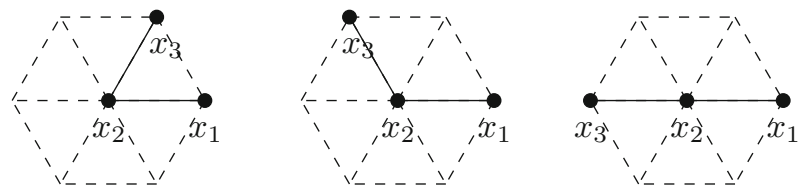

Fig. 9. The three different (up to rotation and reflection) possibilities of paths of length 3

Note that $M^{+}=\emptyset$ or $M^{-}=\emptyset$ if $z^{+}=\mathbf{0}$ or $z^{-}=\mathbf{0}$, respectively. Moreover, we point out that $M^{ \pm}$are in general not subsets of $Q_{T}^{v}(y)$. We illustrate $M^{ \pm} \cap Q_{T}^{v}$ in Fig. 8.

Lemma 5.3. (Simple paths in maximal components) Let $\gamma=\left(x_{1}, \ldots, x_{k}\right)$ be a simple path in $X$ with $x_{1}, x_{k} \in M^{+}$(or both in $M^{-}$) such that $x_{2}, \ldots, x_{k-1} \notin M^{+}$ (or $x_{2}, \ldots, x_{k-1} \notin M^{-}$, respectively). Then $k \geqq 4$.

Proof. Let $\gamma$ be as in the statement, without restriction with $x_{1}, x_{k} \in M^{+}$. Recall that $M^{+} \subset \mathscr{L}\left(z^{+}\right)$. If we had $k=3$, then we would necessarily get $x_{2} \in \mathscr{L}\left(z^{+}\right)$, as well, see Fig. 9. This, however, contradicts the choice of the maximal component $M^{+}$. In fact, also $M^{+} \cup\left\{x_{2}\right\}$ would be a strongly connected set.

Proof. Without restriction we assume $z^{+} \neq z^{-}$. The proof strategy is as follows: we first show that $X$ consists of at most two connected components which contain the lower and the upper part of the boundary, respectively (Step 1). We are then left with at most two connected components which contain the maximal components $M^{ \pm}$defined in (5.5). Then, we prove that these components $M^{ \pm}$do not contain holes. This ensures that $\partial M^{ \pm} \cap Q_{T}^{v}(y)$ are simple paths (Step 2). Finally, we show that there are no parts of $X$ that may be connected to $M^{ \pm}$, but that are not subsets of the upper and lower lattice $\mathscr{L}\left(z^{ \pm}\right)$(Step 3$)$. Steps $1-3$ are proved by contradiction, that is, we suppose that $X$ did not satisfy the abovementioned properties and then 
we show that the configuration can be modified in such a way that the energy strictly decreases. Some technical estimates are given in Steps 4-5.

Fix $z^{ \pm} \in \mathcal{Z}, v \in \mathbb{S}^{1}, T>0$, and $y \in \mathbb{R}^{2}$. Denote by $X \subset \mathbb{R}^{2}$ a minimizer of (5.3). Without loss of generality we assume that

$$
X \subset\left\{x \in \overline{\left(Q_{T}^{v}(y)\right)_{1}}: \mathcal{N}(x) \cap Q_{T}^{v}(y) \neq \emptyset\right\} \cup \partial_{1}^{+} Q_{T}^{v}(y) \cup \partial_{1}^{-} Q_{T}^{v}(y) .
$$

In particular, we have $X=\mathscr{L}\left(z^{ \pm}\right)$on $\partial_{1}^{ \pm} Q_{T}^{v}(y)$. By $M^{ \pm}$we denote its maximal upper and lower component, respectively, given by (5.5). (Recall that $M^{+}=\varnothing$ or $M^{-}=\varnothing$ if $z^{+}=\mathbf{0}$ or $z^{-}=\mathbf{0}$.) Without restriction we assume that $z^{ \pm}=$ $\left(\theta^{ \pm}, \tau^{ \pm}, 1\right)$. Otherwise, we apply all arguments just to the component $z^{ \pm}$with $z^{ \pm} \neq \mathbf{0}$.

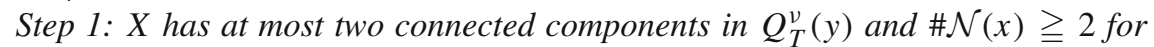
all $x \in X \cap Q_{T}^{v}(y)$. First, we observe that the maximal components $M^{+}$and $M^{-}$ are either contained in one single or in two different connected components of $X$. Assume by contradiction that the configuration $X$ consists of more than the (at most two) connected components containing $M^{ \pm}$. Then we can remove the other connected components not containing $M^{ \pm}$and obtain a new configuration which has strictly less energy and the same boundary data as $X$. This follows directly from the definition of the energy in (2.3).

Moreover, if there exists $x^{\prime} \in X$ such that $\# \mathcal{N}\left(x^{\prime}\right) \leqq 1$, then we can consider the configuration $X \backslash\left\{x^{\prime}\right\}$ to obtain a configuration with strictly less energy since, by (2.3), we have

$$
E_{1}\left(X, Q_{T}^{v}(y)\right)=\frac{1}{2} \sum_{x \in X \cap Q_{T}^{v}(y)}(6-\# \mathcal{N}(x)) \geqq E_{1}\left(X \backslash\left\{x^{\prime}\right\}, Q_{T}^{v}(y)\right)+2 .
$$

Step 2: $\partial M^{ \pm}$is a simple path. In this step, we show that each of the sets $\partial M^{ \pm}$ defined in (5.2) is a simple path in $X$ joining the lateral faces of $Q_{T}^{v}(y)$. More precisely, let

$$
\begin{aligned}
H_{v^{\perp},-}^{T}(y) & :=\left\{x \in \mathbb{R}^{2}:\left\langle(x-y), v^{\perp}\right\rangle<-T / 2\right\} \text { and } H_{v^{\perp},+}^{T}(y) \\
& =\left\{x \in \mathbb{R}^{2}:\left\langle(x-y), v^{\perp}\right\rangle \geqq T / 2\right\} .
\end{aligned}
$$

Then there are $v_{-}^{ \pm} \in M^{ \pm} \cap H_{v^{\perp},-}^{T}(y)$ and $v_{+}^{ \pm} \in M^{ \pm} \cap H_{v^{\perp},+}^{T}(y)$ such that $\left\{v_{-}^{ \pm}, v_{+}^{ \pm}\right\} \cup$ $\partial M^{ \pm}$is a simple path with first element $v_{-}^{ \pm}$and last element $v_{+}^{ \pm}$.

To prove this, we color each (closed) equilateral triangle of sidelength 1 all of whose corners are contained in $M^{ \pm}$in dark/light gray, respectively, see Fig. 8. We first show that there are no cycles in $\partial M^{ \pm}$. Since $M^{ \pm}$is strongly connected, this also yields that the colored regions inside $Q_{T}^{v}(y)$ are simply connected and that $\partial M^{ \pm}$ lies on the boundary of the respective colored region. Assume by contradiction that there exists a cycle $p=\left(v_{1}, \ldots, v_{n}\right) \subset M^{ \pm}$with $v_{n}=v_{1}$. Denote by $\operatorname{int}(p)$ the interior connected component of the curve

$$
p_{\gamma}=\bigcup_{i=1}^{n-1}\left[v_{i} ; v_{i+1}\right]
$$


see Fig. 8. Now define

$$
\tilde{X}= \begin{cases}\mathscr{L}\left(z^{+}\right) & \text {in } \operatorname{int}(p) \\ X & \text { otherwise }\end{cases}
$$

Since we did not change the neighborhood of each atom $x \in Q_{T}^{v}(y) \backslash \overline{\operatorname{int}(p)}$, we obtain by (2.3) and Lemma 3.1(iv)

$$
\begin{aligned}
E_{1}\left(\tilde{X}, Q_{T}^{v}(y)\right) & =E_{1}(\tilde{X}, \overline{\operatorname{int}(p)})+E_{1}\left(\tilde{X}, Q_{T}^{v}(y) \backslash \overline{\operatorname{int}(p)}\right) \\
& <E_{1}(X, \overline{\operatorname{int}(p)})+E_{1}\left(X, Q_{T}^{v}(y) \backslash \overline{\operatorname{int}(p)}\right)=E_{1}\left(X, Q_{T}^{v}(y)\right),
\end{aligned}
$$

where we have used that $\# \mathcal{N}(x)=6$ for all $x \in \tilde{X} \cap \operatorname{int}(p)$ and that every $x \in p$ has at least as many bonds in $\tilde{X}$ as in $X$, while for at least one $x \in p$ the number of bonds has increased. We have constructed a configuration $\tilde{X}$ with strictly less energy and the same boundary data as $X$. This contradicts the fact that $X \subset \mathbb{R}^{2}$ is a minimizer of (5.3), and shows that there are no such cycles in $M^{ \pm}$.

We next show that even the complement of each colored region inside $Q_{T}^{v}(y)$ is connected. If this were not the case, without restriction we assume for contradiction that there are $v, w \in M^{+} \cap H_{v^{\perp},+}^{T}(y)$ such that there is a simple path with first element $v$, last element $w$, and intermediate elements in $\partial M^{+}$, whose bonds together with a segment in $\partial Q_{T}^{v}(y)$ bound a region free of dark triangles. By the boundary conditions, we can suppose that $6 \geqq\langle v, v\rangle>\langle w, v\rangle \geqq-6$, see also Fig. 8. We extend it to a cycle $p$ by placing additional atoms in $\mathscr{L}\left(z^{+}\right) \cap \overline{\left(Q_{T}^{v}(y)\right)_{\varepsilon}} \cap H_{\nu^{\perp},+}^{T}(y)$. Our assumptions on $X$ specified in (5.6) and Step 1 guarantee that each point in $\mathscr{L}\left(z^{+}\right)$on or inside of $p$ has distance at least 1 to every atom of the connected component of $X$ that contains $M^{-}$. Now let

$$
\tilde{X}= \begin{cases}\mathscr{L}\left(z^{+}\right) & \text {in } \overline{\operatorname{int}(p)} \\ X & \text { in } \mathbb{R}^{2} \backslash \overline{\operatorname{int}(p)} \\ \varnothing & \text { otherwise }\end{cases}
$$

In a fashion similiar to before we get $E_{1}\left(\tilde{X}, Q_{T}^{v}(y)\right)<E_{1}\left(X, Q_{T}^{v}(y)\right)$, which also shows that this situation does not occur. We conclude that each $M^{ \pm}$is strongly connected and both the dark and the light colored areas have connected complements relative to $Q_{T}^{v}(y)$.

We claim that $\partial M^{ \pm}$has to be a simple path. Assume by contradiction that this were not the case, for example, for $M^{+}$. Then, since $\partial M^{+}$lies on the boundary of the region in dark gray being the union of triangles, we find $x \in \partial M^{+}$which is a corner of exactly two of these triangles and these triangles share only $x$ as a common point, see Fig. 10. Since $\partial M^{+}$does not contain cycles, we find $x^{+}, x^{-} \in \mathcal{N}(x)$ such that each path in $M^{+}$connecting $x^{+}$with $x^{-}$contains $x$. This, however, contradicts the strong connectedness of $M^{+}$, and shows that $\partial M^{+}$is a simple path. This concludes Step 2.

Step 3: Comparison with subsets of the lattice. Our goal is to show that there holds $X \subset \mathscr{L}\left(z^{+}\right) \cup \mathscr{L}\left(z^{-}\right)$. Recalling the definition of $M^{ \pm}$in (5.5), it thus suffices to show that removing the connected components of $\left(X \cap Q_{T}^{v}(y)\right) \backslash\left(M^{+} \cup M^{-}\right)$ 


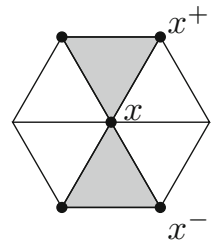

Fig. 10. A point $x \in \partial M^{ \pm}$that would make $\partial M^{ \pm}$a non-simple path

would strictly decrease the energy which clearly contradicts the assumption that $X$ is a minimizer. (Recall that we have already reduced to the case that $X$ consists of at most two connected components. Note, however, that $\left(X \cap Q_{T}^{v}(y)\right) \backslash\left(M^{+} \cup M^{-}\right)$ might consist of more connected components.)

This will conclude the proof of the statement: it shows that the minimizer $X$ is indeed a subset of $\mathscr{L}\left(z^{+}\right) \cup \mathscr{L}\left(z^{-}\right)$. Moreover, the property that $\partial M^{ \pm} \cap Q_{T}^{v}(y)$ are simple paths joining the lateral faces of $Q_{T}^{v}(y)$ has already been addressed in Step 2. Finally, we observe that $\# \mathcal{N}(x) \leqq 5$ for all $x \in \partial M^{ \pm}$. In fact, $\# \mathcal{N}(x)=6$ for some $x \in \partial M^{ \pm}$would entail $\{x\} \cup \overline{\mathcal{N}}(x) \subset M^{ \pm}$as $M^{ \pm} \subset \mathscr{L}\left(z^{ \pm}\right)$is the maximal component. This contradicts (5.2).

Now, consider a connected component $X^{\prime}$ of $\left(X \cap Q_{T}^{v}(y)\right) \backslash\left(M^{+} \cup M^{-}\right)$. We want to prove that

$$
E_{1}\left(X, Q_{T}^{v}(y)\right) \geqq E_{1}\left(X \backslash X^{\prime}, Q_{T}^{v}(y)\right)+1 .
$$

We first introduce some further notation. By $\Gamma^{ \pm} \subset \partial M^{ \pm}$we denote the smallest connected sets $\Gamma^{ \pm} \supset \mathcal{N}\left(X^{\prime}\right) \cap M^{ \pm}$, where we define $\mathcal{N}\left(X^{\prime}\right):=\bigcup_{x \in X^{\prime}} \mathcal{N}(x) \backslash X^{\prime}$ Define $\Gamma:=\Gamma^{+} \cup \Gamma^{-}$and $X_{\Gamma}:=X^{\prime} \cup \Gamma$. Note that both $\Gamma^{-}$and $\Gamma^{+}$are simple paths in $X$ since $\partial M^{ \pm}$are simple paths, see Fig. 11. For $x \in X_{\Gamma}$, we introduce the internal and external neighborhoods by

$$
\mathcal{N}_{i}(x)=\mathcal{N}(x) \cap X_{\Gamma}, \quad \mathcal{N}_{e}(x)=\mathcal{N}(x) \backslash X_{\Gamma},
$$

that is, the set of neighbors inside and outside of $X_{\Gamma}$, respectively. Note that $X_{\Gamma}$ is connected. Its reduced bond graph is delimited by a finite union of disjoint cycles. We denote by $\partial X_{\Gamma}$ the union of these cycles and by $d=\# \partial X_{\Gamma}$ its cardinality. (The notation is unrelated to (5.2).) We further define

$$
\begin{aligned}
& f_{j}=\# j \text {-gons of } X_{\Gamma}, \quad f=\sum_{j} f_{j}, \quad \eta=\eta\left(X_{\Gamma}\right), \quad n_{\Gamma}=\# \Gamma, \quad n=\# X_{\Gamma}, \\
& b_{\Gamma}=\#\{\{x, y\}: x, y \in \Gamma, y \in \mathcal{N}(x)\}, \quad b=\#\left\{\{x, y\}: x, y \in X_{\Gamma}, y \in \mathcal{N}(x)\right\}, \\
& b_{\text {ac }}=\#\left\{\{x, y\} \text { acyclic }: x, y \in X_{\Gamma}, y \in \mathcal{N}(x)\right\}
\end{aligned}
$$

where $\eta$ was introduced in (5.4). Note that $f$ corresponds to the number of faces both in the bond graph and in the reduced bond graph of $X_{\Gamma}$. We will see that it holds that

$$
2+d+2 b_{\mathrm{ac}}+\eta \geqq 3 n_{\Gamma}-b_{\Gamma} .
$$


(a)

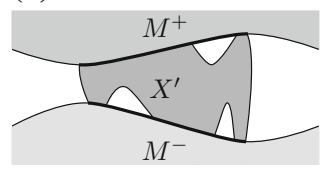

(c)

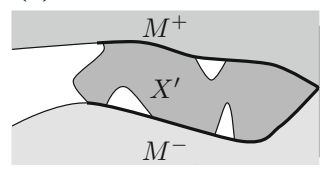

(b)
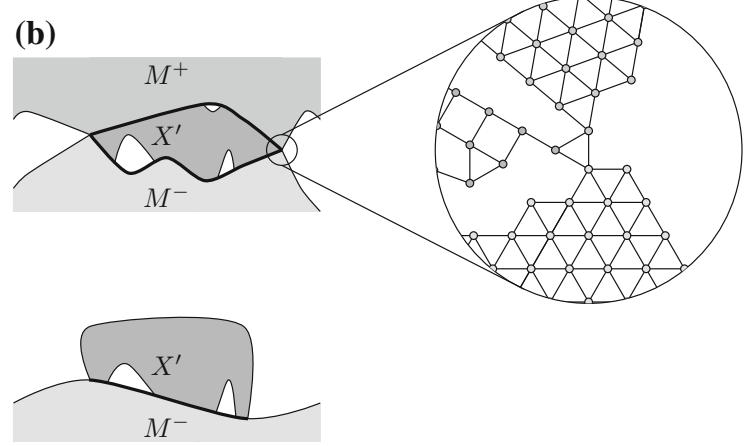

Fig. 11. The different possibilities of $X^{\prime}$ touching $M^{ \pm}$corresponding to case (a) on the top left, case (b) on the top right, and case (c) in the two bottom pictures. $M^{+}$is always depicted in gray, $M^{-}$in light gray, and $X^{\prime}$ in dark gray. $\Gamma^{+}$and $\Gamma^{-}$are depicted by the bold black lines

We defer the proof of (5.10) to Steps 4-5 below and proceed to prove (5.7).

Since in the passage from $X$ to $X \backslash X^{\prime}$ the neighborhood of atoms outside $X_{\Gamma}$ is left unchanged and for atoms in $\Gamma$ the neighbors outside of $X_{\Gamma} \backslash \Gamma$ remain, in view of (2.3), we need to check that

$$
\frac{1}{2} \sum_{x \in X_{\Gamma}}(6-\# \mathcal{N}(x)) \geqq \frac{1}{2} \sum_{x \in \Gamma}\left(6-\left(\# \mathcal{N}_{e}(x)+\#(\mathcal{N}(x) \cap \Gamma)\right)+1 .\right.
$$

We can count the faces to obtain

$$
2 b-d-2 b_{\mathrm{ac}}=\sum_{j \geqq 3} j f_{j}=\eta+3 f .
$$

Indeed, the first identity follows from the fact that in the summation all bonds contained in the union of cycles delimiting the reduced bond graph of $X_{\Gamma}$ are counted only once, the acyclic bonds are not counted, and all other cyclic bonds are counted twice. The second identity follows from (5.4). As the bond graph is planar and connected, we can apply Euler's formula (omitting the exterior face) to get $n-b+f=1$. Then, by (5.10) and (5.12) we derive

$$
3 n-b \geqq 3 n_{\Gamma}-b_{\Gamma}+1 .
$$

By the definitions in (5.8)-(5.9) and the facts that $\sum_{x \in X_{\Gamma}} \# \mathcal{N}_{i}(x)=2 b$, $\sum_{x \in \Gamma} \#(\mathcal{N}(x) \cap \Gamma)=2 b_{\Gamma}$ this implies

$$
\frac{1}{2} \sum_{x \in X_{\Gamma}}\left(6-\# \mathcal{N}_{i}(x)\right) \geqq \frac{1}{2} \sum_{x \in \Gamma}(6-\#(\mathcal{N}(x) \cap \Gamma))+1 .
$$

Now we note that $\# \mathcal{N}(x)-\# \mathcal{N}_{e}(x)=\# \mathcal{N}_{i}(x)$ for $x \in \Gamma$ and $\mathcal{N}(x)=\mathcal{N}_{i}(x)$ for $x \in X_{\Gamma} \backslash \Gamma$, see (5.8). This along with (5.13) shows the desired estimate (5.11). To conclude the proof, it remains to show (5.10). 
Step 4: Proof of (5.10). Recall that $\Gamma$ consists of the two simple paths $\Gamma^{+}$and $\Gamma^{-}$. We need to distinguish three cases:
(a) $\Gamma$ is not connected,
(b) $\Gamma$ is a cycle, (c) $\Gamma$ is a simple path.

Since $\Gamma^{ \pm}$are simple paths, and the bond graph of $X^{\prime}$ is planar and connected, we see that these are all possibilities that may occur, see Fig. 11 for an illustration of the different cases. At this point, we also use that $\Gamma^{ \pm}$are the smallest connected sets with $\Gamma^{ \pm} \supset \mathcal{N}\left(X^{\prime}\right) \cap M^{ \pm}$and $\Gamma^{ \pm} \subset \partial M^{ \pm}$, where $\partial M^{ \pm}$is a simple path connecting $H_{v^{\perp},-}^{T}(y) \cap \mathscr{L}\left(z^{ \pm}\right)$and $H_{v^{\perp},+}^{T}(y) \cap \mathscr{L}\left(z^{ \pm}\right)$.

First of all, we observe that

$$
\text { Case (a): } n_{\Gamma} \leqq b_{\Gamma}+2, \quad \text { Case (b): } n_{\Gamma} \leqq b_{\Gamma}, \quad \text { Case (c): } n_{\Gamma} \leqq b_{\Gamma}+1 \text {. }
$$

This is due to the fact that the bond graph of $\Gamma$ contains $\Gamma^{ \pm}$and a simple path containing $k$ bonds consists of $k+1$ atoms, and in a cycle the number of bonds equals the number of atoms. (As there may be more bonds present if there are triangles in the bond graph, we get inequalities.)

Using (5.14), it suffices to prove

$$
d+2 b_{\mathrm{ac}}+\eta \geqq \begin{cases}2 n_{\Gamma} & \text { in case }(\mathrm{a}), \\ 2 n_{\Gamma}-2 & \text { in case (b), } \\ 2 n_{\Gamma}-1 & \text { in case (c), }\end{cases}
$$

where $d, \eta, n_{\Gamma}$, and $b_{\text {ac }}$ are defined in (5.9). This will rely on the estimate

$$
\eta \geqq n_{\Gamma}-2 \text {. }
$$

We first show (5.15) in the three cases and defer the proof of (5.16) to Step 5. Observe that if a connected component $\tilde{\Gamma}$ of $\Gamma$ satisfies $\tilde{\Gamma} \not \subset \partial X_{\Gamma}$, then $\# \tilde{\Gamma}=1$ and $\tilde{\Gamma}$ connects to $X^{\prime}$ by one acyclic bond. This follows from the observation that, whenever $x \in \tilde{\Gamma}$ satisfies $\mathcal{N}(x) \cap X_{\Gamma} \geqq 2$, then $x$ lies on a cycle in $X_{\Gamma}$ and thus, as an element of $\Gamma$, is contained in $\partial X_{\Gamma}$.

Case (a): Suppose first $\Gamma \subset \partial X_{\Gamma}$. Since $\partial X_{\Gamma}$ is a disjoint union of cycles and $\Gamma$ consists of two simple paths, we get $\#\left(\partial X_{\Gamma} \backslash \Gamma\right) \geqq 2$. In fact, if $\Gamma^{+}$and $\Gamma^{-}$intersect the same cycle of $\partial X_{\Gamma}$, this follows from the fact that $\Gamma^{+} \cup \Gamma^{-}$is not connected. If $\Gamma^{+}$and $\Gamma^{-}$intersect different cycles of $\partial X_{\Gamma}$, it suffices to use that $\Gamma^{ \pm}$are not cycles. This shows $d \geqq n_{\Gamma}+2$. Then (5.16) implies (5.15). If $\Gamma^{-} \subset \partial X_{\Gamma}, \Gamma^{+} \not \subset \partial X_{\Gamma}$, then, as before, $\#\left(\partial X_{\Gamma} \backslash \Gamma^{-}\right) \geqq 1$ and thus $d \geqq \# \Gamma^{-}+1$. The observation below (5.16) gives $\# \Gamma^{+}=1$ and $b_{\text {ac }} \geqq 1$, so particularly $d \geqq n_{\Gamma}$. Then again (5.16) implies (5.15). The case $\Gamma^{-} \not \subset \partial X_{\Gamma}, \Gamma^{+} \subset \partial X_{\Gamma}$ is analogous. Finally, if $\Gamma^{-}, \Gamma^{+} \not \subset \partial X_{\Gamma}$, then $n_{\Gamma}=2$ and $b_{\mathrm{ac}} \geqq 2$ since $\Gamma^{-}$and $\Gamma^{+}$cannot be connected to $X^{\prime}$ by the same (acyclic) bond. This proves (5.15).

Case (b): Since $\Gamma$ is a cycle, we get $\Gamma \subset \partial X_{\Gamma}$. Thus, we obtain $n_{\Gamma} \leqq d$ and (5.16) yields (5.15).

Case (c): Suppose first that $\Gamma \subset \partial X_{\Gamma}$. Since $\Gamma$ is not a cycle and $\partial X_{\Gamma}$ is a union of cycles, we get \# $\left(\partial X_{\Gamma} \backslash \Gamma\right) \geqq 1$. This implies $d \geqq n_{\Gamma}+1$. Then (5.16) again yields (5.15). If $\Gamma \not \subset \partial X_{\Gamma}$, then $n_{\Gamma}=1$ and $b_{\mathrm{ac}} \geqq 1$, from which (5.15) follows. 
Step 5: Proof of (5.16). It remains to check (5.16). To this end, we classify the polygons in the (reduced) bond graph of $X_{\Gamma}$ in the following way: for $k \geqq 1$, we set

$$
\partial \text {-k-gon }=\left\{P \text { polygon in } X_{\Gamma}: \#(P \cap \Gamma)=k\right\} \quad \text { and } \quad \partial \text {-gon }=\bigcup_{k \geqq 1} \partial-k \text {-gon, }
$$

and define $D_{k}=\# \partial-k$-gon. In order to estimate the cardinality of $P \in \partial$-k-gon, we introduce the following condition:

there exist $x_{+} \in M^{+} \cap P$ and $x_{-} \in\left(M^{-} \backslash M^{+}\right) \cap P \quad$ with $\quad\left|x_{+}-x_{-}\right|=1$.

We claim that always, \#P $\geqq k+1$, while if (5.17) does not hold then it holds that $\# P \geqq k+2$.

To see the first claim we note that clearly \#P $P k$. If $\# P=k$, then $P \subset \Gamma$ and $\Gamma$ is a cycle, hence $P=\Gamma$. But then all bonds connecting $\Gamma$ and $X^{\prime}$ are acyclic. As observed below (5.16), this entails $\# \Gamma=1$ which, however, is not possible in case $\Gamma$ is a cycle.

Assume now (5.17) does not hold. First, suppose that $P \cap \Gamma \subset M^{+}$or $P \cap \Gamma \subset$ $M^{-}$. If $k=1$, the statement $\# P \geqq k+2$ is clear as $\# P \geqq 3$. If $k \geqq 2$, we can choose a simple path in $P$ such that only the first and the last atom lie in $M^{+}$ (or $M^{-}$, respectively). The statement then follows from Lemma 5.3. On the other hand, if $P \cap\left(M^{+} \backslash M^{-}\right) \neq \emptyset$ and $P \cap\left(M^{-} \backslash M^{+}\right) \neq \emptyset$, then there exist two simple paths contained in $P$ joining $M^{+} \backslash M^{-}$and $M^{-} \backslash M^{+}$. Since (5.17) does not hold, each of these two paths contains an atom that is not contained in $\Gamma$. This implies $\# P \geqq k+2$.

We are now in a position to prove (5.16). By the definition of $\eta$ and the cardinality estimate for $\partial-k$-gons we obtain

$$
\begin{aligned}
\eta=\sum_{j \geqq 3} f_{j}(j-3) & \geqq \sum_{k \geqq 1} D_{k}(k+2-3)-N \\
& \geqq \sum_{k \geqq 1} D_{k}(k-1)- \begin{cases}0 & \text { in case (a), } \\
2 & \text { in case (b), } \\
1 & \text { in case (c), }\end{cases}
\end{aligned}
$$

where $N$ denotes the number of $\partial$-gons satisfying case (5.17). We used that: in case (a) we have $N=0$ since otherwise $\Gamma$ would be connected, in case (b) the fact that $X^{\prime}$ is connected and the planarity of the bond graph imply that $N \leqq 2$, and in case (c) we get $N \leqq 1$ since $\Gamma$ is a simple path. Finally, we claim that

$$
\sum_{k \geqq 1} D_{k}(k-1) \geqq \begin{cases}n_{\Gamma}-2 & \text { in case (a), } \\ n_{\Gamma} & \text { in case }(\mathrm{b}), \\ n_{\Gamma}-1 & \text { in case }(\mathrm{c}),\end{cases}
$$

Indeed, this follows from the fact that each bond in between two successive atoms $x, y \in \Gamma$ is contained in exactly one $\partial$-gon and $k-1$ estimates from above the 
number of bonds between atoms in $\Gamma \cap P$ whenever $P \in \partial$-k-gon as otherwise $P=\Gamma$ and $\# P=k$ which we have excluded above. (The estimate is strict if $\Gamma \cap P$ is not connected.) By combining (5.18)-(5.19) we obtain (5.16). This concludes the proof.

Proof of Lemma 5.2. Without restriction we assume that $z^{+} \neq z^{-}$. Let $X^{ \pm}$be as in the statement of Lemma 5.1, that is, $X^{ \pm}=M^{ \pm}$. We define

$$
\begin{aligned}
& Y^{+}=X^{+} \backslash\left(\partial X^{+} \cap \partial X^{-}\right) \cup\left\{x \in \partial X^{+} \cap \partial X^{-}: \#\left(\mathcal{N}(x) \cap X^{+}\right) \geqq \#\left(\mathcal{N}(x) \cap X^{-}\right)\right\}, \\
& Y^{-}=X^{-} \backslash\left(\partial X^{+} \cap \partial X^{-}\right) \cup\left\{x \in \partial X^{+} \cap \partial X^{-}: \#\left(\mathcal{N}(x) \cap X^{+}\right)<\#\left(\mathcal{N}(x) \cap X^{-}\right)\right\} .
\end{aligned}
$$

Proof of (i). Property (i) is obviously satisfied by construction.

Proof of (ii). As a preparation, let us note that, if $x \in X^{+} \cap X^{-}$, then $\mathcal{N}(x) \cap X^{+} \cap$ $X^{-}=\emptyset$ since $z^{+} \neq z^{-}$. Moreover, if $x \in X^{+} \cap X^{-} \cap Q_{T}^{v}(y)=\partial X^{+} \cap \partial X^{-}$, then $\# \mathcal{N}(x) \leqq 5$ by Lemma 5.1(ii). Since $X^{ \pm}$is strongly connected, we also have $\#\left(\mathcal{N}(x) \cap X^{\overline{ \pm}}\right) \geqq 2$. Our definition of $Y^{ \pm}$then entails

$$
x \in X^{ \pm} \backslash Y^{ \pm} \Longrightarrow \#\left(\mathcal{N}(x) \cap X^{ \pm}\right)=2 .
$$

This ensures that $Y^{ \pm}=X^{ \pm}=\mathscr{L}\left(z^{ \pm}\right)$on $\partial_{1}^{ \pm} Q_{T}^{v}(y)$. Furthermore, it entails $\partial Y^{ \pm} \subset$ $\partial X^{ \pm}$. Indeed, $y \in \partial Y^{ \pm} \backslash \partial X^{ \pm}$would give $\#\left(\mathcal{N}(y) \cap X^{ \pm}\right)=6$ and $\#\left(\mathcal{N}(y) \cap Y^{ \pm}\right) \leqq$ 5 , that is, there exists $x \in X^{ \pm} \backslash Y^{ \pm}$with $|x-y|=1$. But then $\#(\mathcal{N}(x) \cap \mathcal{N}(y) \cap$ $\left.X^{ \pm}\right)=2$, which yields the contradiction $\#\left(\mathcal{N}(x) \cap X^{ \pm}\right) \geqq 3$.

Proof of (iii). Since $X^{ \pm}$is simply connected and $x \in \partial X^{ \pm} \backslash \partial Y^{ \pm}$is only possible if $\#\left(\mathcal{N}(x) \cap X^{ \pm}\right)=2$ (see (5.20)), we get that $\partial Y^{ \pm}$is a simple path connecting the lateral faces of $Q_{T}^{v}(y)$. More precisely, by Step 2 of the proof of Lemma 5.1, there are $v_{-}^{ \pm} \in X^{ \pm} \cap H_{v^{\perp},-}^{T}(y)$ and $v_{+}^{ \pm} \in X^{ \pm} \cap H_{v^{\perp},+}^{T}(y)$ such that $\left\{v_{-}^{ \pm}, v_{+}^{ \pm}\right\} \cup \partial Y^{ \pm}$is a simple path with first element $v_{-}^{ \pm}$and last element $v_{+}^{ \pm}$. The bonds between any two consecutive atoms in this chain form a polygonal line and we denote by $\alpha(x)$ the (interior) angle it forms at atom $x$.

As the first and the last segments cross the lateral faces of $Q_{T}^{v}(y)$ and $Y^{ \pm}$is strongly connected, we have

$$
\sum_{x \in \partial Y^{ \pm}}(\pi-\alpha(x)) \in \frac{1}{3}\{-2 \pi,-\pi, 0, \pi, 2 \pi\} .
$$

Since $X^{ \pm}$is simply connected, due to (5.20), the same holds true for $Y^{ \pm}$. Hence, $\alpha(x)$ relates to the number of neighbours of $x$ within $Y^{ \pm}$by the formula

$$
\alpha(x)=\frac{1}{3}\left(\#\left(\mathcal{N}(x) \cap Y^{ \pm}\right)-1\right) \pi .
$$

As a consequence we obtain

$$
\left|\sum_{x \in \partial Y^{ \pm}}\left(\#\left(\mathcal{N}(x) \cap Y^{ \pm}\right)-4\right)\right|=\left|\frac{3}{\pi} \sum_{x \in \partial Y^{ \pm}}(\alpha(x)-\pi)\right| \leqq 2 .
$$

This concludes the proof. 
We summarize our main findings on the structure of grain boundaries obtained in the proof of Lemma 5.1 in the following theorem:

Theorem 5.4. (Reduction to subsets of two lattices) Let $z^{+}, z^{-} \in \mathcal{Z}, z^{+} \neq z^{-}$, $v \in \mathbb{S}^{1}, y \in \mathbb{R}^{2}$, and $T>0$. Let $X \subset \mathbb{R}^{2}$ be a minimizer of

$$
\min \left\{E_{1}\left(X, Q_{T}^{v}(y)\right): X=\mathscr{L}\left(z^{ \pm}\right) \text {on } \partial_{1}^{ \pm} Q_{T}^{v}(y)\right\}
$$

Then $X=M^{+} \cup M^{-}$on $Q_{T}^{v}(y)$, where $M^{+}, M^{-}$are the maximal components of $X$, see (5.5). Coloring each (closed) equilateral triangle of sidelength 1 all of whose corners are contained in $M^{ \pm}$in dark/light gray, yields two simply connected plain regions containing $\partial_{1}^{ \pm} Q_{T}^{v}(y)$, respectively, whose boundary part inside of $Q_{T}^{v}(y)$ is given by a simple path of atoms.

\section{Characterization of Solid-Vacuum/Solid-Solid Interactions}

This section is devoted to establish a relation between the cell formula $\Phi$ defined in (4.1) and the density $\varphi_{\text {hex }}$ given in (2.18). In particular, we will analyze the situation where the two lattices $\mathscr{L}\left(z^{+}\right)$and $\mathscr{L}\left(z^{-}\right)$, which determine the admissible configurations at the boundary, allow for touching points, that is, atoms $x^{+} \in$ $\mathscr{L}\left(z^{+}\right)$and $x^{-} \in \mathscr{L}\left(z^{-}\right)$with $\left|x^{+}-x^{-}\right|=1$. We start by formulating the two results of this section.

Lemma 6.1. (Relation of $\Phi$ and $\varphi_{\mathrm{hex}}$ ) There exists a universal constant $C>0$ such that for each $v \in \mathbb{S}^{1}$ and for every sequence of centers $\left\{y_{T}\right\}_{T}$ the following properties hold:

(i) If $z^{+}=(\theta, \tau, 1) \in \mathcal{Z}$ and $z^{-}=\mathbf{0}$ or if $z^{+}=\mathbf{0}$ and $z^{-}=(\theta, \tau, 1) \in \mathcal{Z}$, there holds for all $T>0$

$$
\mid \frac{1}{T} \min \left\{E_{1}\left(X_{T}, Q_{T}^{v}\left(y_{T}\right)\right): X_{T}=\mathscr{L}\left(z^{ \pm}\right) \text {on } \partial_{1}^{ \pm} Q_{T}^{v}\left(y_{T}\right)\right\}-\varphi_{\text {hex }}\left(e^{-i \theta} v\right) \mid \leqq C / T \text {. }
$$

(ii) For all $z^{+}=\left(\theta^{+}, \tau^{+}, 1\right), z^{-}=\left(\theta^{-}, \tau^{-}, 1\right) \in \mathcal{Z}$ there holds for all $T>0$

$$
\begin{aligned}
& \frac{1}{T} \min \left\{E_{1}\left(X_{T}, Q_{T}^{v}\left(y_{T}\right)\right): X_{T}=\mathscr{L}\left(z^{ \pm}\right) \text {on } \partial_{1}^{ \pm} Q_{T}^{v}\left(y_{T}\right)\right\} \\
& \quad \leqq \varphi_{\text {hex }}\left(e^{-i \theta^{+}} v\right)+\varphi_{\text {hex }}\left(e^{-i \theta^{-}} v\right)+C / T .
\end{aligned}
$$

Moreover, if $z^{+} \neq z^{-}$, then also

$$
\begin{aligned}
& \frac{1}{T} \min \left\{E_{1}\left(X_{T}, Q_{T}^{v}\left(y_{T}\right)\right): X_{T}=\mathscr{L}\left(z^{ \pm}\right) \text {on } \partial_{1}^{ \pm} Q_{T}^{v}\left(y_{T}\right)\right\} \\
& \quad \geqq \frac{1}{2} \varphi_{\text {hex }}\left(e^{-i \theta^{+}} v\right)+\frac{1}{2} \varphi_{\text {hex }}\left(e^{-i \theta^{-}} v\right)-C / T .
\end{aligned}
$$


Note that this lemma indeed provides a relation between $\varphi_{\text {hex }}$ and the density $\Phi$ since

$\Phi\left(z^{+}, z^{-}, v\right) \leqq \liminf _{T \rightarrow+\infty} \frac{1}{T} \min \left\{E_{1}\left(X_{T}, Q_{T}^{v}\left(y_{T}\right)\right): X_{T}=\mathscr{L}\left(z^{ \pm}\right)\right.$on $\left.\partial_{1}^{ \pm} Q_{T}^{v}\left(y_{T}\right)\right\}$

for all $z^{ \pm} \in \mathcal{Z}, v \in \mathbb{S}^{1}$, and all $\left\{y_{T}\right\}_{T}$. We point out that the energy density $\varphi_{\text {hex }}$ has already been identified in [3,20]. In our exposition, once the technical result about reduction to two lattices (see Lemma 5.1) has been achieved, the proof of Lemma 6.1(i) is rather simple compared to [20, Theorem 2.2]. In addition, this version with convergence rate is a novel result and is needed in order to prove Proposition 2.2.

The next lemma is a refinement which addresses the question under which conditions on the difference of the rotation angles $\theta^{+}-\theta^{-}$equality holds in (ii). To formulate this statement, recall $\omega=\frac{1}{2}+\frac{i}{2} \sqrt{3}$ from Section 2.2. We introduce the set of good angles, denoted by $\mathcal{G}_{\mathbb{A}}$, as the angles $\theta \in \mathbb{A}$ which can be written as

$$
e^{i \theta}=\frac{v_{1}}{v_{2}}, \quad \text { with } v_{1}, v_{2} \in \mathscr{L} \backslash\{0\} .
$$

Here, the division of $v_{1}, v_{2} \in \mathbb{C}$ has to be understood in the sense of complex numbers. I.e., such angles correspond to rotations which transform one lattice point into another one. Note that $\mathcal{G}_{\mathbb{A}}$ is clearly countable. From an algebraic standpoint, our notion of $\mathcal{G}_{\mathbb{A}}$ coincides with those angles $\theta$ such that $e^{i \theta}$ is a fraction of the commutative ring $\mathscr{L}$.

Lemma 6.2. (Touching lattices) Let $z^{ \pm}=\left(\theta^{ \pm}, \tau^{ \pm}, 1\right) \in \mathcal{Z}$ be such that

$$
\Phi\left(z^{+}, z^{-}, v\right) \leqq \varphi_{\mathrm{hex}}\left(e^{-i \theta^{-}} \nu\right)+\varphi_{\mathrm{hex}}\left(e^{-i \theta^{+}} \nu\right)-\eta
$$

for an $\eta>0$. Then, there exists an optimal sequence $\left\{X_{T}\right\}_{T}$ for $\Phi\left(z^{+}, z^{-}, v\right)$, see (5.1), such that for all $T>0$ large enough, there holds $X_{T} \subset \mathscr{L}\left(z_{T}^{+}\right) \cup \mathscr{L}\left(z_{T}^{-}\right)$, where $z_{T}^{ \pm}=\left(\theta_{T}^{ \pm}, \tau_{T}^{ \pm}, 1\right) \in \mathcal{Z}$, and the rotation angles satisfy

$$
\theta_{T}^{+}-\theta_{T}^{-}=\theta^{+}-\theta^{-} \in \mathcal{G}_{\mathbb{A}} \text { for all } T>0 .
$$

More precisely, $e^{i\left(\theta^{+}-\theta^{-}\right)}=v_{1} / v_{2}$ for lattice vectors $v_{1}, v_{2} \in \mathscr{L} \backslash\{0\}$ with $\left|v_{1}\right|,\left|v_{2}\right|$ $\leqq C_{\eta}$, where $C_{\eta}>0$ only depends on $\eta$.

Condition (6.3) means that the surface energy between sub-lattices of $\mathscr{L}\left(z^{+}\right)$ and $\mathscr{L}\left(z^{-}\right)$can be strictly less than the sum of the surface energies corresponding to each lattice interacting with the vacuum. This indicates that there are many atoms (in a certain sense) in $\mathscr{L}\left(z^{+}\right)$with distance 1 to atoms in $\mathscr{L}\left(z^{-}\right)$. Therefore, we speak of lattices which have "touching points". The lemma shows two properties of optimal sequences: (i) they can be chosen as a subset of two lattices only, cf. also Lemma 5.1, (ii) the difference of the corresponding rotation angles is constant and lies in $\mathcal{G}_{\mathbb{A}}$.

We now proceed with the proofs of the two lemmas. 
Proof of Lemma 6.1. For the whole proof, we fix $v \in \mathbb{S}^{1}$ and a sequence of centers $\left\{y_{T}\right\}_{T}$.

Proof of (i). Let $z=(\theta, \tau, 1) \in \mathcal{Z} \backslash\{\mathbf{0}\}$. We only prove the result for $z^{+}=z$ and $z^{-}=\mathbf{0}$ since the argumentation for the reflected boundary conditions is the same. We obtain the statement by showing separately the two inequalities, where one is proved by a slicing argument and the other one in a constructive way.

Step 1: First inequality. The goal of this step is to prove

$$
\frac{1}{T} \min \left\{E_{1}\left(X_{T}, Q_{T}^{v}\left(y_{T}\right)\right): X_{T}=\mathscr{L}\left(z^{ \pm}\right) \text {on } \partial_{1}^{ \pm} Q_{T}^{v}\left(y_{T}\right)\right\} \geqq \varphi_{\text {hex }}\left(e^{-i \theta} v\right)-C / T \text {. }
$$

Consider $X_{T} \subset \mathbb{R}^{2}$ satisfying $X_{T}=\mathscr{L}(z)$ on $\partial_{1}^{+} Q_{T}^{v}\left(y_{T}\right), X_{T}=\mathscr{L}(\mathbf{0})=\varnothing$ on $\partial_{1}^{-} Q_{T}^{v}\left(y_{T}\right)$, and

$$
E_{1}\left(X_{T}, Q_{T}^{v}\left(y_{T}\right)\right)=\min \left\{E_{1}\left(\tilde{X}_{T}, Q_{T}^{v}\left(y_{T}\right)\right): \tilde{X}_{T}=\mathscr{L}\left(z^{ \pm}\right) \text {on } \partial_{1}^{ \pm} Q_{T}^{v}\left(y_{T}\right)\right\} .
$$

By Lemma 5.1, we get that $X_{T} \subset \mathscr{L}(z)=e^{i \theta}(\mathscr{L}+\tau)$. Recall the definition $\omega=\frac{1}{2}+\frac{i}{2} \sqrt{3}$. We now perform a slicing argument: for $k \in\{1,2,3\}$, we define for each $\mu \in \mathbb{R}$

$$
I_{k}(\mu):=\left\{\lambda e^{i \theta} \omega^{k}+\mu e^{i \theta}\left(\omega^{k}\right)^{\perp}: \lambda \in \mathbb{R}\right\}
$$

the line in lattice direction $e^{i \theta} \omega^{k}$ passing through the line $\mathbb{R} e^{i \theta}\left(\omega^{k}\right)^{\perp}$ at point $\mu e^{i \theta}\left(\omega^{k}\right)^{\perp}$. We set

$$
\mathcal{I}_{k}=\left\{\mu \in \mathbb{R}: I_{k}(\mu) \cap \mathscr{L}(z) \neq \emptyset, I_{k}(\mu) \cap\left[y_{T}-\frac{T}{2} v^{\perp} ; y_{T}+\frac{T}{2} v^{\perp}\right]\right\} .
$$

Due to the boundary conditions, up to a bounded number of times independent of both $v$ and $T$, for each $\mu \in \mathcal{I}_{k}$ we find $x \in X_{T} \subset \mathscr{L}(z)$ such that $x+e^{i \theta} \omega^{k} \notin X_{T}$ or $x-e^{i \theta} \omega^{k} \notin X_{T}$. (Note that a bounded number of lattice lines, independent of $T$, in direction $e^{i \theta} \omega^{k}$ and passing through $\left[y_{T}-\frac{T}{2} v^{\perp} ; y_{T}+\frac{T}{2} v^{\perp}\right]$ does not intersect $\partial_{1}^{+} Q_{T}^{v}\left(y_{T}\right)$.) By (2.3) this yields

$$
E_{1}\left(X_{T}, Q_{T}^{v}\left(y_{T}\right)\right) \geqq \sum_{k=1}^{3} \# \mathcal{I}_{k}-C
$$

for a constant $C>0$ independent of $T$. It remains to estimate \# $\mathcal{I}_{k}$. For $\mu \in \mathbb{R}$ such that $I_{k}(\mu) \cap \mathscr{L}(z) \neq \emptyset$, we get $I_{k}(\mu \pm \sqrt{3} / 2) \cap \mathscr{L}(z) \neq \emptyset$ and $I_{k}\left(\mu^{\prime}\right) \cap \mathscr{L}(z)=\varnothing$ for all $\mu^{\prime} \in(\mu-\sqrt{3} / 2, \mu+\sqrt{3} / 2) \backslash\{\mu\}$. Finally, we have

$$
\mathcal{L}^{1}\left(\Pi_{k}\left(\left[y_{T}-\frac{T}{2} v^{\perp} ; y_{T}+\frac{T}{2} v^{\perp}\right]\right)\right)=T\left|\left\langle v, e^{i \theta} \omega^{k}\right\rangle\right|,
$$

where $\Pi_{k}$ denotes the orthogonal projection onto $\mathbb{R} e^{i \theta}\left(\omega^{k}\right)^{\perp}$. We therefore obtain

$$
\# \mathcal{I}_{k} \geqq \frac{2 T}{\sqrt{3}}\left|\left\langle\nu, e^{i \theta} \omega^{k}\right\rangle\right|-C=\frac{2 T}{\sqrt{3}}\left|\left\langle e^{-i \theta} \nu, \omega^{k}\right\rangle\right|-C .
$$


By (2.18) and (6.6)-(6.7) we conclude

$$
\frac{1}{T} E_{1}\left(X_{T}, Q_{T}^{v}\left(y_{T}\right)\right) \geqq \frac{2}{\sqrt{3}} \sum_{k=1}^{3}\left|\left\langle e^{-i \theta} v, \omega^{k}\right\rangle\right|-C / T=\varphi_{\mathrm{hex}}\left(e^{-i \theta} v\right)-C / T .
$$

This along with (6.5) shows the first inequality.

Step 2: Second inequality. The goal of this step is to prove

$$
\frac{1}{T} \min \left\{E_{1}\left(X_{T}, Q_{T}^{v}\left(y_{T}\right)\right): X_{T}=\mathscr{L}\left(z^{ \pm}\right) \text {on } \partial_{1}^{ \pm} Q_{T}^{v}\left(y_{T}\right)\right\} \leqq \varphi_{\text {hex }}\left(e^{-i \theta} v\right)+C / T .
$$

This is achieved by constructing an explicit competitor for the minimization problem: we define $X_{T}^{+}$by

$$
X_{T}^{+}= \begin{cases}\mathscr{L}(z) & \text { in }\left\{x:\left\langle x-y_{T}, \nu\right\rangle \geqq 5\right\}, \\ \varnothing & \text { otherwise }\end{cases}
$$

that is, $X_{T}^{+}$is a (discrete version of a) half space. We directly see that $X_{T}^{+}=\mathscr{L}(z)$ on $\partial_{1}^{+} Q_{T}^{\nu}\left(y_{T}\right)$ and $X_{T}^{+}=\varnothing$ on $\partial_{1}^{-} Q_{T}^{\nu}\left(y_{T}\right)$. To estimate its energy, we start by observing that for this choice of $X_{T}^{+}$equality holds in (6.6) with $\mathcal{I}_{k}$ as defined above, up to an error of order $\mathrm{O}(1)$. Indeed, if $x \in \mathscr{L}(z) \backslash X_{T}^{+}$, then either $x+\lambda e^{i \theta} \omega^{k} \notin X_{T}^{+}$ for all $\lambda \in \mathbb{N}$ or $x-\lambda e^{i \theta} \omega^{k} \notin X_{T}^{+}$for all $\lambda \in \mathbb{N}$. Then, the equalities in (6.6) and (6.7) along with (2.18) yield

$$
\frac{1}{T} E_{1}\left(X_{T}^{+}, Q_{T}^{v}\left(y_{T}\right)\right) \leqq \frac{2}{\sqrt{3}} \sum_{k=1}^{3}\left|\left\langle e^{-i \theta} v, \omega^{k}\right\rangle\right|+C / T=\varphi_{\mathrm{hex}}\left(e^{-i \theta} v\right)+C / T .
$$

This shows (6.8). For purposes of the proof of (ii) below, we note that construction (6.9) with $-v$ in place of $v$ can be applied to obtain a configuration $X_{T}^{-} \subset \mathbb{R}^{2}$ with $X_{T}^{-}=\mathscr{L}(z)$ on $\partial_{1}^{-} Q_{T}^{v}\left(y_{T}\right)$ and $X_{T}^{-}=\emptyset$ on $\partial_{1}^{+} Q_{T}^{v}\left(y_{T}\right)$ which satisfies (6.10).

Proof of (ii). Fix $z^{+}=\left(\theta^{+}, \tau^{+}, 1\right) \in \mathcal{Z}$ and $z^{-}=\left(\theta^{-}, \tau^{-}, 1\right) \in \mathcal{Z}$. We show the first inequality by an explicit construction. The second one is obtained with the help of Lemma 5.2.

Step 1: First inequality. We define $X_{T}=X_{T}^{+} \cup X_{T}^{-}$, where

$$
\begin{aligned}
& X_{T}^{+}=\left\{\begin{array}{ll}
\mathscr{L}\left(z^{+}\right) & \text {in }\left\{x:\left\langle x-y_{T}, v\right\rangle \geqq 5\right\}, \\
\varnothing & \text { otherwise. }
\end{array},\right. \\
& X_{T}^{-}= \begin{cases}\mathscr{L}\left(z^{-}\right) & \text {in }\left\{x:\left\langle x-y_{T}, v\right\rangle \leqq-5\right\}, \\
\varnothing & \text { otherwise. }\end{cases}
\end{aligned}
$$

Then, $X_{T}$ clearly satisfies the boundary conditions $X_{T}=\mathscr{L}\left(z^{ \pm}\right)$on $\partial_{1}^{ \pm} Q_{T}^{v}\left(y_{T}\right)$ and by repeating the reasoning in (6.10) we find that

$$
\frac{1}{T} E_{T}\left(X_{T}, Q_{T}^{\nu}\left(y_{T}\right)\right)=\frac{1}{T}\left(E_{1}\left(X_{T}^{+}, Q_{T}^{\nu}\left(y_{T}\right)\right)+E_{1}\left(X_{T}^{-}, Q_{T}^{\nu}\left(y_{T}\right)\right)\right)
$$




$$
\leqq \varphi_{\mathrm{hex}}\left(e^{-i \theta^{+}} v\right)+\varphi_{\mathrm{hex}}\left(e^{-i \theta^{-}} v\right)+C / T \text {. }
$$

Step 2: Second inequality. Consider $X_{T} \subset \mathbb{R}^{2}$ satisfying $X_{T}=\mathscr{L}\left(z^{ \pm}\right)$on $\partial_{1}^{ \pm} Q_{T}^{v}\left(y_{T}\right)$ and

$$
E_{1}\left(X_{T}, Q_{T}^{v}\left(y_{T}\right)\right)=\min \left\{E_{1}\left(\tilde{X}_{T}, Q_{T}^{v}\left(y_{T}\right)\right): \tilde{X}_{T}=\mathscr{L}\left(z^{ \pm}\right) \text {on } \partial_{1}^{ \pm} Q_{T}^{\nu}\left(y_{T}\right)\right\} .
$$

By Lemmas 5.1 and 5.2 there holds $X_{T}=X_{T}^{+} \cup X_{T}^{-}=Y_{T}^{+} \dot{\cup} Y_{T}^{-}$on $Q_{T}^{\nu}(y)$, where $Y_{T}^{ \pm}=\mathscr{L}\left(z^{ \pm}\right)$on $\partial_{1}^{ \pm} Q_{T}^{v}\left(y_{T}\right)$ and

$$
\left|\sum_{x \in \partial Y_{T}^{ \pm}} \#\left(\mathcal{N}(x) \cap Y_{T}^{ \pm}\right)-4 \# \partial Y_{T}^{ \pm}\right| \leqq 2 .
$$

Since $\# \mathcal{N}(x) \leqq 5$ for any $x \in \partial Y_{T}^{ \pm}\left(\subset \partial X_{T}^{ \pm}\right)$, we get

$$
\frac{1}{2} \sum_{x \in Y_{T}^{ \pm} \cap Q_{T}^{v}\left(y_{T}\right)}(6-\# \mathcal{N}(x)) \geqq \frac{1}{2} \# \partial Y_{T}^{ \pm} \geqq \frac{1}{4} \sum_{x \in \partial Y_{T}^{ \pm}}\left(6-\#\left(\mathcal{N}(x) \cap Y_{T}^{ \pm}\right)\right)-1 / 2 .
$$

So observing that $Y_{T}^{ \pm}$is a competitor in (Step 1 of) (i) above and using that $Y_{T}^{+} \cap$ $Y_{T}^{-} \cap Q_{T}^{v}\left(y_{T}\right)=\emptyset$, we find that

$$
\begin{aligned}
\frac{1}{T} E_{1}\left(X_{T}, Q_{T}^{v}\left(y_{T}\right)\right) & \geqq \frac{1}{2 T} E_{1}\left(Y_{T}^{+}, Q_{T}^{v}\left(y_{T}\right)\right)+\frac{1}{2 T} E_{1}\left(Y_{T}^{-}, Q_{T}^{v}\left(y_{T}\right)\right)-1 / T \\
& \geqq \frac{1}{2} \varphi_{\text {hex }}\left(e^{-i \theta^{+}} \nu\right)+\frac{1}{2} \varphi_{\text {hex }}\left(e^{-i \theta^{-}} \nu\right)-C / T
\end{aligned}
$$

This concludes the proof.

Proof of Lemma 6.2. Let $\left\{X_{T}\right\}_{T}$ be an optimal sequence for $\Phi\left(z^{+}, z^{-}, v\right)$ and denote by $\left\{y_{T}\right\}_{T}$ the corresponding centers of the cubes. Due to Lemma 5.1, we may without restriction assume that $X_{T}=X_{T}^{+} \cup X_{T}^{-}$, for sub-configurations $X_{T}^{ \pm}$ satisfying $X_{T}^{ \pm} \subset \mathscr{L}\left(z_{T}^{ \pm}\right)$, where $z_{T}^{ \pm}=\left(\theta_{T}^{ \pm}, \tau_{T}^{ \pm}, 1\right) \rightarrow z^{ \pm}=\left(\theta^{ \pm}, \tau^{ \pm}, 1\right)$ as $T \rightarrow+\infty$. Moreover, the sets $\partial X^{ \pm}$defined in (5.2) are connected, and there holds $X_{T}=\mathscr{L}\left(z_{T}^{ \pm}\right)$on $\partial_{1}^{ \pm} Q_{T}^{v}\left(y_{T}\right)$. In what follows, we fix a subsequence (not relabeled) such that by (6.3) we have

$$
\varphi_{\text {hex }}\left(e^{-i \theta^{+}} \nu\right)+\varphi_{\text {hex }}\left(e^{-i \theta^{-}} \nu\right)-\lim _{T \rightarrow+\infty} \frac{1}{T} E_{1}\left(X_{T}, Q_{T}^{\nu}\left(y_{T}\right)\right) \geqq \eta>0 .
$$

Our strategy to show (6.4) lies in proving

$$
e^{i\left(\theta_{T}^{+}-\theta_{T}^{-}\right)}=\frac{v_{T}^{+}}{v_{T}^{-}} \quad \text { with } \quad v_{T}^{+}, v_{T}^{-} \in \mathscr{L} \quad \text { satisfying } \quad\left|v_{T}^{+}\right|=\left|v_{T}^{-}\right| \leqq C_{\eta}
$$

for all $T$ sufficiently large, where $C_{\eta}$ only depends on $\eta$. From this estimate, the statement in (6.4) easily follows. In fact, given (6.12), since $\mathscr{L}$ is a discrete set and $\theta_{T}^{ \pm} \rightarrow \theta^{ \pm}, e^{i\left(\theta_{T}^{+}-\theta_{T}^{-}\right)}=v_{T}^{+} / v_{T}^{-}$is eventually constant and we find $\theta^{+}-\theta^{-}=$ $\theta_{T}^{+}-\theta_{T}^{-} \in \mathcal{G}_{\mathbb{A}}$ for all $T$ large enough. 
Let us come to the proof of (6.12). Recall by Lemma 5.1 that $X_{T}$ is contained in the two components $X_{T}^{+}$and $X_{T}^{-}$. We further define the set of touching points

$$
\begin{aligned}
& \mathcal{T}_{T}^{+}=\left\{x \in X_{T}^{+}: \exists y \in X_{T}^{-} \text {such that }|x-y|=1\right\}, \\
& \mathcal{T}_{T}^{-}=\left\{x \in X_{T}^{-}: \exists y \in X_{T}^{+} \text {such that }|x-y|=1\right\} .
\end{aligned}
$$

Note that $\mathcal{T}_{T}^{ \pm} \subset \bigcup_{x \in \partial X_{T}^{ \pm}}(\{x\} \cup \mathcal{N}(x))$, see definition (5.2). ( $\mathcal{T}_{T}^{ \pm} \backslash \partial X_{T}^{ \pm} \neq \varnothing$ is possible if $X_{T}^{+} \cap X_{T}^{-} \neq \emptyset$.) By (2.2) we also observe that

$$
\# \mathcal{T}_{T}^{+} / 6 \leqq \# \mathcal{T}_{T}^{-} \leqq 6 \# \mathcal{T}_{T}^{+} .
$$

We start with a brief outline of the proof. Steps 1-4 are devoted to some preliminary estimates: we first show that the cardinality of the sets $\partial X_{T}^{ \pm}$and $\mathcal{T}_{T}^{ \pm}$scales like $T$ by providing a lower bound for $\mathcal{T}_{T}^{ \pm}$(Step 1) and an upper bound for $\partial X_{T}^{ \pm}$(Step 2). Then we show that, for the majority of points in $\mathcal{T}_{T}^{ \pm}$, neighborhoods contain many points of $\partial X_{T}^{ \pm}$(Step 3) and also elements of $\mathcal{T}_{T}^{ \pm}$(Step 4). Based on this, we can find quadrilaterals consisting of two points in $\mathcal{T}_{T}^{+}$and two points in $\mathcal{T}_{T}^{-}$where two sides have length 1 and the other two sides are parallel to lattice vectors of the form $e^{i \theta_{T}^{+}} w_{T}^{+}$and $e^{i \theta_{T}^{-}} w_{T}^{-}$, respectively, for some $w_{T}^{+}, w_{T}^{-} \in \mathscr{L}$ with controlled norm. From this, (6.12) can be derived (Step 5 and Step 6).

Step 1: Cardinality of touching points. We show $\# \mathcal{T}_{T}^{ \pm} \geqq \frac{\eta}{22} T$ for $T$ large enough. By (2.2), (2.3), and the fact that $X_{T}^{ \pm}=\mathscr{L}\left(z_{T}^{ \pm}\right)$on $\partial_{1}^{ \pm} Q_{T}^{v}\left(y_{T}\right)$, we obtain

$$
\begin{aligned}
E_{1}\left(X_{T}, Q_{T}^{v}\left(y_{T}\right)\right) \geqq & \frac{1}{2} \sum_{x \in X_{T}^{+} \cap Q_{T}^{v}\left(y_{T}\right)}\left(6-\#\left(\mathcal{N}(x) \cap X_{T}^{+}\right)\right) \\
+ & \frac{1}{2} \sum_{\substack{x \in X_{T}^{-} \cap Q_{T}^{v}\\
}}\left(6-\#\left(y_{T}\right)\right. \\
& -3\left(\# \mathcal{T}_{T}^{+}+\# \mathcal{T}_{T}^{-}\right),
\end{aligned}
$$

and therefore,

$$
3\left(\# \mathcal{T}_{T}^{+}+\# \mathcal{T}_{T}^{-}\right) \geqq E_{1}\left(X_{T}^{+}, Q_{T}^{v}\left(y_{T}\right)\right)+E_{1}\left(X_{T}^{-}, Q_{T}^{v}\left(y_{T}\right)\right)-E_{1}\left(X_{T}, Q_{T}^{v}\left(y_{T}\right)\right) .
$$

We note by the definition of $X_{T}$ that the subconfigurations $X_{T}^{+}$and $X_{T}^{-}$are competitors for the minimization problems appearing in Lemma 6.1(i). Dividing by $T$ and passing to the lim inf along $T \rightarrow+\infty$, by (6.11) we therefore conclude

$$
\liminf _{T \rightarrow+\infty} \frac{1}{T}\left(\# \mathcal{T}_{T}^{+}+\# \mathcal{T}_{T}^{-}\right) \geqq \eta / 3 \text {. }
$$

This yields $\lim \inf _{T \rightarrow+\infty} \frac{1}{T} \# \mathcal{T}_{T}^{ \pm} \geqq \frac{\eta}{21}$ by (6.13), and concludes Step 1 .

Step 2: A priori bound on the length of the boundaries. We claim that for $T>0$ large enough the boundaries $\partial X_{T}^{ \pm} \subset Q_{T}^{v}\left(y_{T}\right)$ (cf. (5.2)) satisfy

$$
\#\left(\partial X_{T}^{+} \cup \partial X_{T}^{-}\right) \leqq 8 T .
$$


In fact, by Lemma 5.1(ii) there holds $\# \mathcal{N}(x) \leqq 5$ for all $x \in \partial X_{T}^{ \pm}$and therefore for $T$ sufficiently large we get by (2.3), (6.11), and the fact that $\left\|\varphi_{\text {hex }}\right\|_{L^{\infty}\left(\mathbb{S}^{1}\right)}=2$ $(\operatorname{see}(2.18))$

$$
\begin{aligned}
\#\left(\partial X_{T}^{+} \cup \partial X_{T}^{-}\right) & \leqq \\
x \in X_{T} \cap Q_{T}^{v}\left(y_{T}\right) & (6-\# \mathcal{N}(x))=2 E_{1}\left(X_{T}, Q_{T}^{v}\left(y_{T}\right)\right) \\
& \leqq 2 T\left(\varphi_{\mathrm{hex}}\left(e^{-i \theta^{+}} \nu\right)+\varphi_{\mathrm{hex}}\left(e^{-i \theta^{-}} \nu\right)\right) \leqq 8 T .
\end{aligned}
$$

Step 3: Atomic density lower bound for $\partial X_{T}^{ \pm}$. We claim that there exists a universal $0<c<1$ such that, for all $T>r \geqq 1$, we have

$$
\#\left(\partial X_{T}^{ \pm} \cap B_{r}(x)\right) \geqq c r \quad \text { for all } x \in \mathbb{R}^{2} \text { with } \operatorname{dist}\left(x, \partial X_{T}^{ \pm}\right) \leqq 1 .
$$

To prove this estimate we assume without restriction that $T>3 r$. Due to Lemma 5.1(ii), $\partial X_{T}^{ \pm}$is connected and $\partial X_{T}^{ \pm} \backslash B_{r}(x) \neq \varnothing$. Therefore, there has to exist a simple path in $\partial X_{T}^{ \pm}$that connects some atom in $\partial X_{T}^{ \pm} \backslash B_{r}(x)$ with an atom in $\overline{B_{1}(x)}$ and has at least $c r$ atoms inside $B_{r}(x)$.

Step 4: Bounded gap between points in $\mathcal{T}_{T}^{ \pm}$. Given $R>0$, we introduce the set of $R$-isolated points by

$$
\mathcal{I}_{T, R}^{ \pm}:=\left\{x \in \mathcal{T}_{T}^{ \pm}: B_{R}(x) \cap \mathcal{T}_{T}^{ \pm} \subset \overline{B_{2}(x)}\right\} .
$$

We claim that there exists a universal $\bar{c}>0$ such that for $R=\bar{c} / \eta$ and all $T$ sufficiently large

$$
\# \mathcal{I}_{T, R}^{ \pm} \leqq \# \mathcal{T}_{T}^{ \pm} / 2
$$

To see this, note that due to (6.14), (6.15) for $r=R / 2$ (use that $\operatorname{dist}\left(x, \partial X_{T}^{ \pm}\right) \leqq 1$ for all $\left.x \in \mathcal{T}_{T}^{ \pm}\right)$and Step 1 we have

$$
\# \mathcal{I}_{T, R}^{ \pm} \leqq \frac{2}{c R} \sum_{x \in \mathcal{I}_{T, R}^{ \pm}} \#\left(\partial X_{T}^{ \pm} \cap B_{R / 2}(x)\right) \leqq \frac{C}{c R} \# \partial X_{T}^{ \pm} \leqq \frac{C}{c R} T \leqq \frac{C}{c \bar{c}} \# \mathcal{T}_{T}^{ \pm},
$$

where $C>0$ denotes a universal constant varying from step to step. Here, in the second step we accounted for possible multiple counting by using that, due to the definition of $\mathcal{I}_{T, R}^{ \pm}$, the intersection $B_{R / 2}(x) \cap B_{R / 2}(y), x, y \in \mathcal{I}_{T, R_{T}}^{ \pm}$, can be non-empty only if $|x-y| \leqq 2$. The assertion follows if $\bar{c}$ is chosen big enough.

Step 5: Bounded gap between pairs of points having the same relative position. We choose two arbitrary lattice vectors $\xi_{1}, \xi_{2}$ satisfying $e^{-i \theta_{T}^{-}} \xi_{1}, e^{-i \theta_{T}^{+}} \xi_{2} \in B_{2 R} \cap$ $(\mathscr{L} \backslash\{0\})$ with $R>0$ given by Step 4. Define

$$
\begin{aligned}
\mathcal{D}_{T}^{\xi_{1}, \xi_{2}}= & \left\{\left(x_{1}, y_{1}\right) \in \mathcal{T}_{T}^{-} \times \mathcal{T}_{T}^{-}: \text {there exist } x_{2}, y_{2} \in \mathcal{T}_{T}^{+}\right. \text {such that } \\
& \left.\left|x_{1}-x_{2}\right|=1,\left|y_{1}-y_{2}\right|=1 \text { and } x_{1}-y_{1}=\xi_{1}, x_{2}-y_{2}=\xi_{2}\right\} .
\end{aligned}
$$

The set consists of pairs $\left(x_{1}, y_{1}\right)$ in $\mathcal{T}_{T}^{-}$whose difference is $\xi_{1}$ and which have corresponding neighbors in $\mathcal{T}_{T}^{+}$with difference $\xi_{2}$. 
We observe by (6.16) that for $x_{1} \in \mathcal{T}_{T}^{-} \backslash \mathcal{I}_{T, R}^{-}$we find $\xi_{1} \in B_{R} \cap e^{i \theta_{T}^{-}} \mathscr{L}$ with $\left|\xi_{1}\right|>2$ and $y_{1} \in \mathcal{T}_{T}^{-}$such that $x_{1}-y_{1}=\xi_{1}$. We denote the corresponding neighbors in $\mathcal{T}_{T}^{+}$by $x_{2}$ and $y_{2}$, respectively. Since $x_{2}, y_{2} \in e^{i \theta_{T}^{+}}\left(\mathscr{L}+\tau_{T}^{+}\right)$and $\left|x_{1}-y_{1}\right|=\left|x_{2}-y_{2}\right|=1$, we find $\xi_{2} \in B_{2 R} \cap e^{i \theta_{T}^{+}} \mathscr{L}$ such that $x_{2}-y_{2}=\xi_{2}$. Clearly, $\xi_{2} \neq 0$ as $\left|\xi_{1}\right|>2$. This discussion along with (6.17) implies

$$
\frac{1}{2} \# \mathcal{T}_{T}^{-} \leqq \#\left(\mathcal{T}_{T}^{-} \backslash \mathcal{I}_{T, R}^{-}\right) \leqq \sum_{\left(\xi_{1}, \xi_{2}\right)} \# \mathcal{D}_{T}^{\xi_{1}, \xi_{2}}
$$

where the sum runs over all pairs $\left(\xi_{1}, \xi_{2}\right)$ with $e^{-i \theta_{T}^{-}} \xi_{1}, e^{-i \theta_{T}^{+}} \xi_{2} \in B_{2 R} \cap(\mathscr{L} \backslash\{0\})$. Choose $\left(\zeta_{1}^{T}, \zeta_{2}^{T}\right) \in\left(B_{2 R} \cap e^{i \theta_{T}^{-}}(\mathscr{L} \backslash\{0\})\right) \times\left(B_{2 R} \cap e^{i \theta_{T}^{+}}(\mathscr{L} \backslash\{0\})\right)$ such that $\# \mathcal{D}_{T}^{\zeta_{1}^{T}}, \zeta_{2}^{T} \geqq \# \mathcal{D}_{T}^{\xi_{1}, \xi_{2}}$ for all $\left(\xi_{1}, \xi_{2}\right) \in\left(B_{2 R} \cap e^{i \theta_{T}^{-}}(\mathscr{L} \backslash\{0\})\right) \times\left(B_{2 R} \cap e^{i \theta_{T}^{+}}(\mathscr{L} \backslash\{0\})\right)$. Then, (6.18) and the fact that the number of pairs $e^{-i \theta_{T}^{-}} \xi_{1}, e^{-i \theta_{T}^{+}} \xi_{2} \in B_{2 R} \cap$ $(\mathscr{L} \backslash\{0\})$ is controlled by $C R^{4}$ yield

$$
\# \mathcal{T}_{T}^{-} \leqq C R^{4} \# \mathcal{D}_{T}^{\zeta_{1}^{T}, \zeta_{2}^{T}}
$$

for a universal $C>0$. We write $\mathcal{D}_{T}^{\zeta_{1}^{T}, \zeta_{2}^{T}}=\left\{x_{j}^{T}, y_{j}^{T}\right\}_{j=1}^{M_{T}}$ for some $M_{T} \in \mathbb{N}$. We claim that there is a universal $c^{\prime}>0$ such that for $\varrho=c^{\prime} \eta^{-5}$

there exist $j, k, l \in\left\{1, \ldots, M_{T}\right\}$ pairwise distinct such that $x_{k}^{T}, x_{l}^{T} \in B_{\varrho}\left(x_{j}^{T}\right)$.

Assume that, on the contrary, $\varrho$ is such that each $B_{\varrho}\left(x_{k}^{T}\right) \backslash\left\{x_{k}^{T}\right\}$ contains at most one point $\left\{x_{j}^{T}\right\}_{j=1}^{M_{T}}$. Then, it is elementary to see that we can choose $\left\{\tilde{x}_{j}^{T}\right\}_{j=1}^{\left\lceil M_{T} / 2\right\rceil} \subset$ $\left\{x_{j}^{T}\right\}_{j=1}^{M_{T}}$ such that $B_{\varrho / 2}\left(\tilde{x}_{j}^{T}\right) \cap B_{\varrho / 2}\left(\tilde{x}_{k}^{T}\right)=\emptyset$ for $j, k \in\left\{1, \ldots,\left\lceil M_{T} / 2\right\rceil\right\}, j \neq k$. This along with (6.14), (6.15), and $2\left\lceil M_{T} / 2\right\rceil \geqq \# \mathcal{D}_{T}^{\zeta_{1}^{T}, \zeta_{2}^{T}}$ implies

$$
\# \mathcal{D}_{T}^{\zeta_{1}^{T}, \zeta_{2}^{T}} \leqq 2\left\lceil\frac{M_{T}}{2}\right\rceil \leqq \frac{4}{c \varrho} \sum_{j=1}^{\left\lceil M_{T} / 2\right\rceil} \#\left(\partial X_{T}^{-} \cap B_{\varrho / 2}\left(\tilde{x}_{j}^{T}\right)\right) \leqq \frac{4}{c \varrho} \# \partial X_{T}^{-} \leqq \frac{32 T}{c \varrho}
$$

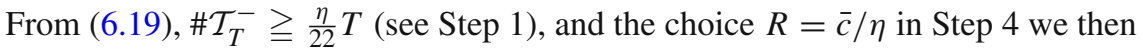
get $\varrho \leqq c^{\prime} \eta^{-5} / 2$ for a universal $c^{\prime}>0$. The assertion of (6.20) is thus guaranteed for $\varrho=c^{\prime} \eta^{-5}$. This concludes Step 5 .

Step 6: Conclusion. We denote the three atoms identified in (6.20) by $x_{1}^{1}, x_{1}^{2}, x_{1}^{3}$ (for convenience, we use a different notation and labeling), and denote by $y_{1}^{1}, y_{1}^{2}, y_{1}^{3}$ the corresponding points such that $\left(x_{1}^{j}, y_{1}^{j}\right) \in \mathcal{D}_{T}^{\zeta_{1}^{T}, \zeta_{2}^{T}}$ for $j \in\{1,2,3\}$. In particular, recall that

$$
\left|x_{1}^{1}-x_{1}^{2}\right|, \quad\left|x_{1}^{1}-x_{1}^{3}\right|, \quad\left|x_{1}^{2}-x_{1}^{3}\right| \leqq 2 \varrho .
$$

By the definition of $\mathcal{D}_{T}^{\zeta_{1}^{T}, \zeta_{2}^{T}}$, there exist $\left(x_{2}^{1}, y_{2}^{1}\right),\left(x_{2}^{2}, y_{2}^{2}\right),\left(x_{2}^{3}, y_{2}^{3}\right)$ such that $\mid x_{1}^{j}-$ $x_{2}^{j}|=| y_{1}^{j}-y_{2}^{j} \mid=1, \zeta_{1}^{T}=x_{1}^{j}-y_{1}^{j}$, and $\zeta_{2}^{T}=x_{2}^{j}-y_{2}^{j}$ for $j \in\{1,2,3\}$. 

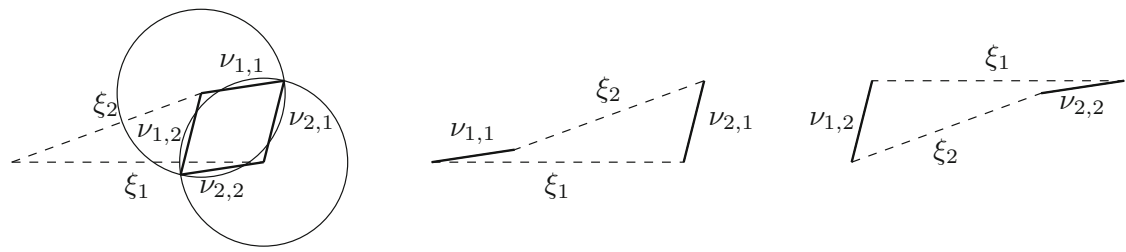

Fig. 12. The two possible quadrilaterals in Step 6 , where $\xi_{1}, \xi_{2}$ are given unlike vectors and $v_{1,1}, v_{2,1}, v_{1,2}, v_{2,2}$ denote the possible sides of length 1

Now for each $j$, the four points $\left\{x_{1}^{j}, x_{2}^{j}, y_{2}^{j}, y_{1}^{j}\right\}$ form a quadrilateral (possibly selfintersecting) with two edges of length one and two edges oriented in $\zeta_{1}^{T}$ and $\zeta_{2}^{T}$, respectively. Now there are two cases to consider: (a) $\zeta_{1}^{T}=\zeta_{2}^{T}$ and (b) $\zeta_{1}^{T} \neq \zeta_{2}^{T}$. Case (a): We have that $x_{1}^{1}-y_{1}^{1}=x_{2}^{1}-y_{2}^{1}$, where $x_{1}^{1}-y_{1}^{1}=e^{i \theta_{T}^{-}} v_{1}$ and $x_{2}^{1}-y_{2}^{1}=$ $e^{i \theta_{T}^{+}} v_{2}$ for $v_{1}, v_{2} \in(\mathscr{L} \backslash\{0\}) \cap B_{2 R}$. Then $e^{i \theta_{T}^{-}} v_{1}=e^{i \theta_{T}^{+}} v_{2}$ and thus (6.12) holds for $v_{T}^{+}=v_{1}$ and $v_{T}^{-}=v_{2}$ with $\left|v_{T}^{+}\right|,\left|v_{T}^{-}\right| \leqq 2 R=2 \bar{c} / \eta$.

Case (b): Note that two of the three quadrilaterals $\left\{x_{1}^{j}, x_{2}^{j}, y_{2}^{j}, y_{1}^{j}\right\}, j \in\{1,2,3\}$, are necessarily translates of each other. In fact, there are only two different quadrilaterals (up to translation) with fixed order of the sides, prescribed side-length 1 of two opposite edges, and prescribed length and orientation of the other two edges, see Fig. 12.

Without restriction, assume that the quadrilaterals for $j=1$ and $j=2$ are translates of each other. Then we get $x_{1}^{1}-x_{2}^{1}=x_{1}^{2}-x_{2}^{2}$. We write $x_{1}^{j}=e^{i \theta_{T}^{-}}\left(b_{1}^{j}+\right.$ $\left.\tau_{T}^{-}\right)$and $x_{2}^{j}=e^{i \theta_{T}^{+}}\left(b_{2}^{j}+\tau_{T}^{+}\right)$for suitable $b_{1}^{j}, b_{2}^{j} \in \mathscr{L}$ for $j \in\{1,2\}$. (Note that the lattice vectors depend on $T$ which we do not include in the notation for convenience.) Then $x_{1}^{1}-x_{2}^{1}=x_{1}^{2}-x_{2}^{2}$ implies $e^{i \theta_{T}^{-}}\left(b_{1}^{1}-b_{1}^{2}\right)=e^{i \theta_{T}^{+}}\left(b_{2}^{1}-b_{2}^{2}\right)$. Since $x_{1}^{1} \neq x_{1}^{2}$ we have $b_{1}^{1}-b_{1}^{2} \neq 0$ and thus also $b_{2}^{1}-b_{2}^{2} \neq 0$, and therefore,

$$
e^{i\left(\theta_{T}^{+}-\theta_{T}^{-}\right)}=\frac{b_{1}^{1}-b_{1}^{2}}{b_{2}^{1}-b_{2}^{2}} .
$$

Due to (6.21), we obtain $\left|b_{1}^{1}-b_{1}^{2}\right|=\left|x_{1}^{1}-x_{1}^{2}\right| \leqq 2 \varrho$ and, since $\left|b_{1}^{1}-b_{1}^{2}\right|=\left|b_{2}^{1}-b_{2}^{2}\right|$, also $\left|b_{2}^{1}-b_{2}^{2}\right| \leqq 2 \varrho$. As we clearly also have $b_{1}^{1}-b_{1}^{2}, b_{2}^{1}-b_{2}^{2} \in \mathscr{L}$, we derive that (6.12) holds for $v_{T}^{+}:=b_{1}^{1}-b_{1}^{2}$ and $v_{T}^{-}:=b_{2}^{1}-b_{2}^{2}$ with $\left|v_{T}^{+}\right|,\left|v_{T}^{-}\right| \leqq 2 \varrho=$ $2 c^{\prime} \eta^{-5}$. As explained below (6.12), (6.12) implies (6.4), and therefore the proof is concluded.

\section{Cell Formula Part II: Relation of Converging and Fixed Boundary Values}

In this final section about cell formulas we show that converging boundary conditions as in the cell formula $\Phi$, see (4.1), can be replaced by fixed boundary values. Moreover, we show Proposition 2.2 and the properties of $\varphi$ stated in Theorem 2.5. We introduce the auxiliary function

$$
\bar{\varphi}\left(z^{+}, z^{-}, v\right):=\liminf _{T \rightarrow+\infty} \frac{1}{T} \inf \left\{E_{1}\left(X_{T}, Q_{T}^{\nu}\left(y_{T}\right)\right): y_{T} \in \mathbb{R}^{2},\right.
$$




$$
\left.X_{T}=\mathscr{L}\left(z^{ \pm}\right) \text {on } \partial_{1}^{ \pm} Q_{T}^{\nu}\left(y_{T}\right)\right\}
$$

for $z^{ \pm} \in \mathcal{Z}$ and $v \in \mathbb{S}^{1}$. The main goal of this section is to prove the following two statements:

Lemma 7.1. For each $z^{+}, z^{-} \in \mathcal{Z}$ and $v \in \mathbb{S}^{1}$ it holds that

$$
\Phi\left(z^{+}, z^{-}, v\right)=\bar{\varphi}\left(z^{+}, z^{-}, v\right) .
$$

Moreover, for $z^{ \pm}=\left(\theta^{ \pm}, \tau^{ \pm}, 1\right) \in \mathcal{Z}$ with $\left\{(x, y) \in \mathscr{L}\left(z^{+}\right) \times \mathscr{L}\left(z^{-}\right):|x-y|=\right.$ $1\}=\emptyset$, we have $\bar{\varphi}\left(z^{+}, z^{-}, v\right)=\varphi_{\text {hex }}\left(e^{-i \theta^{+}} v\right)+\varphi_{\text {hex }}\left(e^{-i \theta^{-}} v\right)$.

Proposition 7.2. For every $z^{+}, z^{-} \in \mathcal{Z}, v \in \mathbb{S}^{1}$, and every sequence $\left\{y_{T}\right\}_{T} \in \mathbb{R}^{2}$ there exists

$$
\bar{\varphi}\left(z^{+}, z^{-}, v\right)=\lim _{T \rightarrow+\infty} \frac{1}{T} \min \left\{E_{1}\left(X_{T}, Q_{T}^{v}\left(y_{T}\right)\right): X_{T}=\mathscr{L}\left(z^{ \pm}\right) \text {on } \partial_{1}^{ \pm} Q_{T}^{v}\left(y_{T}\right)\right\}
$$

and is independent of $\left\{y_{T}\right\}_{T}$. In particular, we get $\varphi \equiv \bar{\varphi}$, and the statement of Proposition 2.2 holds.

We point out that Lemma 7.1, Proposition 7.2, and Lemma 4.1 conclude the proof of Proposition 3.4. Section 7.1 is devoted to the proof of Lemma 7.1. Afterwards, in Section 7.2, we show Proposition 7.2 (which particularly yields Proposition 2.2) and we prove further properties of the density $\varphi$ stated in Theorem 2.5. Then, all proofs of our main results announced in Section 2.3 are concluded.

\subsection{Converging and Fixed Boundary Values}

This subsection is devoted to the proof of Lemma 7.1. By definition it is clear that $\Phi\left(z^{+}, z^{-}, v\right) \leqq \bar{\varphi}\left(z^{+}, z^{-}, v\right)$ for all $z^{+}, z^{-} \in \mathcal{Z}$ and $v \in \mathbb{S}^{1}$. To see (7.2), it therefore suffices to prove the opposite inequality

$$
\Phi\left(z^{+}, z^{-}, v\right) \geqq \bar{\varphi}\left(z^{+}, z^{-}, v\right) .
$$

Moreover, we observe that if $z^{+}=\mathbf{0}$ or $z^{-}=\mathbf{0}$, then Lemma 6.1(i) and the continuity of $\varphi_{\text {hex }}$ imply $\Phi\left(z^{+}, z^{-}, v\right)=\bar{\varphi}\left(z^{+}, z^{-}, v\right)=\varphi_{\text {hex }}\left(e^{-i \theta} v\right)$, where $\theta$ is the angle corresponding to $z^{+}$or $z^{-}$, respectively. Therefore, it suffices to treat the case $z^{ \pm}=\left(\theta^{ \pm}, \tau^{ \pm}, 1\right) \in \mathcal{Z}$. To this end, it is crucial that converging boundary values as in (4.1) can be replaced by fixed ones. We split the analysis into two steps by first addressing the rotations and then the translations. We start with the rotations. In view of Lemma 6.2, we may without restriction assume that $\theta^{+}-\theta^{-} \in \mathcal{G}_{\mathbb{A}}$ since otherwise $\Phi\left(z^{+}, z^{-}, v\right) \geqq \varphi_{\text {hex }}\left(e^{-i \theta^{+}} v\right)+\varphi_{\text {hex }}\left(e^{-i \theta^{-}} v\right)$ and (7.4) follows from Lemma 6.1(ii). Lemma 6.2 already implies that the difference of rotations $\theta_{T}^{+}-\theta_{T}^{-}$ is constant in $T$. The next lemma shows that also $\theta_{T}^{+}$and $\theta_{T}^{-}$can be chosen to be constant. 
Lemma 7.3. (Fixed rotations) Consider $z_{T}^{ \pm}=\left(\theta_{T}^{ \pm}, \tau_{T}^{ \pm}, 1\right) \in \mathcal{Z}$ such that $\theta_{T}^{+}-$ $\theta_{T}^{-}=\theta^{+}-\theta^{-}$for all $T>0$ for some $\theta^{+}, \theta^{-} \in \mathbb{A}$ and $\theta_{T}^{ \pm} \rightarrow \theta^{ \pm}$. Let $v \in \mathbb{S}^{1}$. Then, there holds

$$
\begin{aligned}
& \liminf _{T \rightarrow+\infty} \frac{1}{T} \inf \left\{E_{1}\left(X_{T}, Q_{T}^{v}\left(y_{T}\right)\right): y_{T} \in \mathbb{R}^{2}, X_{T}=\mathscr{L}\left(z_{T}^{ \pm}\right) \text {on } \partial_{1}^{ \pm} Q_{T}^{v}\left(y_{T}\right)\right\} \\
& \geqq \liminf _{T \rightarrow+\infty} \frac{1}{T} \inf \left\{E_{1}\left(X_{T}, Q_{T}^{v}\left(y_{T}\right)\right): y_{T} \in \mathbb{R}^{2}, X_{T}=\mathscr{L}\left(\hat{z}_{T}^{ \pm}\right) \text {on } \partial_{1}^{ \pm} Q_{T}^{v}\left(y_{T}\right)\right\},
\end{aligned}
$$

where $\hat{z}_{T}^{ \pm}:=\left(\theta^{ \pm}, \tau_{T}^{ \pm}, 1\right)$.

We defer the proof and proceed with the properties of translations. Again consider $z^{ \pm}=\left(\theta^{ \pm}, \tau^{ \pm}, 1\right) \in \mathcal{Z}$ with $\theta^{+}-\theta^{-} \in \mathcal{G}_{\mathbb{A}}$. Recall by (6.2) that there holds $e^{i\left(\theta^{+}-\theta^{-}\right)}=\frac{v_{1}}{v_{2}}$ for $v_{1}, v_{2} \in \mathscr{L} \cap \mathbb{C}$ with $\left|v_{1}\right|=\left|v_{2}\right|$. We consider the coincidence site lattice

$$
e^{i \theta^{+}} \mathscr{L} \cap e^{i \theta^{-}} \mathscr{L}=\{j a+k b: j, k \in \mathbb{Z}\},
$$

where $a, b \in e^{i \theta^{+}} \mathscr{L} \cap e^{i \theta^{-}} \mathscr{L}$ are spanning vectors of minimal length. Then, for later purposes, we define the fundamental parallelogram of $e^{i \theta^{+}} \mathscr{L} \cap e^{i \theta^{-}} \mathscr{L}$ by

$$
P_{\theta^{+}, \theta^{-}}=\left\{\lambda_{1} a+\lambda_{2} b: 0 \leqq \lambda_{1}<1,0 \leqq \lambda_{2}<1\right\} .
$$

We will use the following uniform closedness property of the set of touching points between sequences of translates of two perfect lattices.

Lemma 7.4. (Closedness of touching points) Consider $z_{n}^{ \pm}=\left(\theta^{ \pm}, \tau_{n}^{ \pm}, 1\right) \in \mathcal{Z}$ for $n \in \mathbb{N}$ and $z^{ \pm}=\left(\theta^{ \pm}, \tau^{ \pm}, 1\right) \in \mathcal{Z}$ such that $\theta^{+}-\theta^{-} \in \mathcal{G}_{\mathbb{A}}$ and $\tau_{n}^{ \pm} \rightarrow \tau^{ \pm}$. For $x \in \mathscr{L}\left(z^{+}\right), y \in \mathscr{L}\left(z^{-}\right)$we set

$$
x_{n}^{+}=x+e^{i \theta^{+}}\left(\tau_{n}^{+}-\tau^{+}\right) \in \mathscr{L}\left(z_{n}^{+}\right), \quad y_{n}^{-}=y+e^{i \theta^{-}}\left(\tau_{n}^{-}-\tau^{-}\right) \in \mathscr{L}\left(z_{n}^{-}\right) .
$$

Then, there is an $n_{0} \in \mathbb{N}$ such that for all $n \geqq n_{0}$ and all $x \in \mathscr{L}\left(z^{+}\right), y \in \mathscr{L}\left(z^{-}\right)$ the following implications are verified:

$$
\begin{aligned}
& \text { (i) }|x-y|<1 \Longrightarrow\left|x_{n}^{+}-y_{n}^{-}\right|<1 \text { and } \\
& \text { (ii) }|x-y|>1 \Longrightarrow\left|x_{n}^{+}-y_{n}^{-}\right|>1 \text {. }
\end{aligned}
$$

In particular, $\left|x_{n}^{+}-y_{n}^{-}\right|=1$ for some $n \geqq n_{0}$ implies $|x-y|=1$.

We again defer the proof and now proceed with the proof of Lemma 7.1.

Proof of Lemma 7.1. Let $z^{+}, z^{-} \in \mathcal{Z}, v \in \mathbb{S}^{1}$. Recalling the discussion at the beginning of the subsection, we note that it suffices to show inequality (7.4). Moreover, we can assume that $z^{ \pm}=\left(\theta^{ \pm}, \tau^{ \pm}, 1\right)$ and that $\theta^{+}-\theta^{-} \in \mathcal{G}_{\mathbb{A}}$.

Let $\left\{X_{T}\right\}_{T}$ be an optimal sequence for $\Phi$ with corresponding centers $\left\{y_{T}\right\}_{T}$ of the cubes, that is,

$$
\liminf _{T \rightarrow+\infty} \frac{1}{T} E_{1}\left(X_{T}, Q_{T}^{\nu}\left(y_{T}\right)\right)=\Phi\left(z^{+}, z^{-}, v\right)<+\infty .
$$


By applying Lemma 6.2, we can suppose that $X_{T}=X_{T}^{+} \cup X_{T}^{-}$with $X_{T}^{ \pm} \subset \mathscr{L}\left(z_{T}^{ \pm}\right)$ and $X_{T}=\mathscr{L}\left(z_{T}^{ \pm}\right)$on $\partial_{1}^{ \pm} Q_{T}^{v}\left(y_{T}\right)$, where $z_{T}^{ \pm}=\left(\theta_{T}^{ \pm}, \tau_{T}^{ \pm}, 1\right) \rightarrow z^{ \pm}$. By (6.4) and Lemma 7.3 we can also assume that $\theta_{T}^{ \pm}=\theta^{ \pm}$for all $T$. We distinguish the two cases (a) $\left\{(x, y) \in \mathscr{L}\left(z^{+}\right) \times \mathscr{L}\left(z^{-}\right):|x-y|=1\right\}=\emptyset$ and (b) $\{(x, y) \in$ $\left.\mathscr{L}\left(z^{+}\right) \times \mathscr{L}\left(z^{-}\right):|x-y|=1\right\} \neq \emptyset$.

Case (a): $\left\{(x, y) \in \mathscr{L}\left(z^{+}\right) \times \mathscr{L}\left(z^{-}\right):|x-y|=1\right\}=\emptyset$. By Lemma 7.4 we can assume that $\left\{(x, y) \in \mathscr{L}\left(z_{T}^{+}\right) \times \mathscr{L}\left(z_{T}^{-}\right):|x-y|=1\right\}=\emptyset$ for all $T$. Thus, we get $\mathcal{N}(x) \cap X_{T}^{-}=\emptyset$ for all $x \in X_{T}^{+}$and viceversa. Therefore, by (2.3) we obtain

$$
\begin{aligned}
\Phi\left(z^{+}, z^{-}, v\right)= & \liminf _{T \rightarrow+\infty} \frac{1}{T} E_{1}\left(X_{T}, Q_{T}^{v}\left(y_{T}\right)\right)=\liminf _{T \rightarrow+\infty}\left(\frac{1}{T} E_{1}\left(X_{T}^{+}, Q_{T}^{v}\left(y_{T}\right)\right)\right. \\
& \left.+\frac{1}{T} E_{1}\left(X_{T}^{-}, Q_{T}^{v}\left(y_{T}\right)\right)\right) .
\end{aligned}
$$

Note that $X_{T}^{ \pm}=\mathscr{L}\left(z_{T}^{ \pm}\right)=\mathscr{L}\left(\theta^{ \pm}, \tau_{T}^{ \pm}, 1\right)$ on $\partial_{1}^{ \pm} Q_{T}^{v}\left(y_{T}\right)$ and $X_{T}^{ \pm}=\emptyset$ on $\partial_{1}^{\mp} Q_{T}^{v}\left(y_{T}\right)$. By Lemma 6.1(i), the energy on each sublattice $X_{T}^{+}$on $X_{T}^{-}$can be estimated separately, and we obtain

$$
\Phi\left(z^{+}, z^{-}, v\right) \geqq \varphi_{\text {hex }}\left(e^{-i \theta^{+}} v\right)+\varphi_{\text {hex }}\left(e^{-i \theta^{-}} v\right) .
$$

Then, Lemma 6.1(ii) and (7.1) imply $\Phi\left(z^{+}, z^{-}, v\right) \geqq \bar{\varphi}\left(z^{+}, z^{-}, v\right)$ and $\bar{\varphi}\left(z^{+}, z^{-}, v\right)$ $=\varphi_{\text {hex }}\left(e^{-i \theta^{+}} v\right)+\varphi_{\text {hex }}\left(e^{-i \theta^{-}} v\right)$. This concludes the proof of (7.4) in case (a). We also point out that the property stated below (7.2) holds. (In case $\theta^{+}-\theta^{-} \notin \mathcal{G}_{\mathbb{A}}$, (7.8) is immediate from (6.3).)

Case (b): $\left\{(x, y) \in \mathscr{L}\left(z^{+}\right) \times \mathscr{L}\left(z^{-}\right):|x-y|=1\right\} \neq \emptyset$. Our goal is to construct a new competitor $\tilde{X}_{T}=\tilde{X}_{T}^{+} \cup \tilde{X}_{T}^{-}$such that $\tilde{X}_{T}^{ \pm} \subset \mathscr{L}\left(z^{ \pm}\right), \tilde{X}_{T}^{ \pm}=\mathscr{L}\left(z^{ \pm}\right)$on $\partial_{1}^{ \pm} Q_{T+22}^{v}\left(y_{T}\right)$, and

$$
E_{1}\left(\tilde{X}_{T}, Q_{T+22}^{v}\left(y_{T}\right)\right) \leqq E_{1}\left(X_{T}, Q_{T}^{v}\left(y_{T}\right)\right)+C .
$$

Once this is established, by (7.1) and (7.7) we clearly get

$$
\begin{aligned}
\Phi\left(z^{+}, z^{-}, v\right) & =\liminf _{T \rightarrow+\infty} \frac{1}{T} E_{1}\left(X_{T}, Q_{T}^{v}\left(y_{T}\right)\right) \geqq \liminf _{T \rightarrow+\infty} \frac{1}{T+22} E_{1}\left(\tilde{X}_{T}, Q_{T+22}^{v}\left(y_{T}\right)\right) \\
& \geqq \bar{\varphi}\left(z^{+}, z^{-}, v\right) .
\end{aligned}
$$

To construct $\tilde{X}_{T}$, we first extend $X_{T}$ to $\hat{X}_{T}$ by

$$
\hat{X}_{T}= \begin{cases}X_{T} & \text { on } Q_{T+10}^{v}\left(y_{T}\right) \backslash A_{T}, \\ \mathscr{L}\left(z_{T}^{ \pm}\right) & \text {on }\left\{x: \pm\left\langle v, x-y_{T}\right\rangle \geqq 2\right\} \cap\left(Q_{T+34}^{v}\left(y_{T}\right) \backslash\left(Q_{T+10}^{v}\left(y_{T}\right) \cup A_{T}\right)\right), \\ \varnothing & \text { on } A_{T} \cup\left(\mathbb{R}^{2} \backslash Q_{T+34}^{v}\left(y_{T}\right)\right),\end{cases}
$$

where $A_{T}=Q_{10}^{v}\left(y_{T}+(T / 2) v^{\perp}\right) \cup Q_{10}^{v}\left(y_{T}-(T / 2) v^{\perp}\right) \cup\left(\left\{\left|x:\left\langle v, x-y_{T}\right\rangle\right|<\right.\right.$ $\left.2\} \backslash Q_{T+10}^{v}\left(y_{T}\right)\right)$. By definition, we get $E_{1}\left(\hat{X}_{T}\right)<+\infty$ since $|x-y| \geqq 1$ for all $x, y \in \hat{X}_{T}, x \neq y$. Note that we can write $\hat{X}_{T}=\hat{X}_{T}^{+} \dot{\cup} \hat{X}_{T}^{-}$, where $\hat{X}_{T}^{ \pm} \subset \mathscr{L}\left(z_{T}^{ \pm}\right)$ and $\hat{X}_{T}^{ \pm}=\mathscr{L}\left(z_{T}^{ \pm}\right)$on $\partial_{1}^{ \pm} Q_{T+22}^{v}\left(y_{T}\right)$. We claim that

$$
E_{1}\left(\hat{X}_{T}, Q_{T+32}^{v}\left(y_{T}\right)\right) \leqq E_{1}\left(X_{T}, Q_{T}^{v}\left(y_{T}\right)\right)+C .
$$


In fact, if there exists $x \in \hat{X}_{T} \cap Q_{T}^{v}\left(y_{T}\right)$ such that $\#\left(\mathcal{N}(x) \cap \hat{X}_{T}\right)<\#\left(\mathcal{N}(x) \cap X_{T}\right)$, then necessarily $x \in\left(A_{T}\right)_{1} \cap Q_{T}^{v}\left(y_{T}\right)$. However, $\mathcal{L}^{2}\left(\left(A_{T} \cap Q_{T}^{v}\left(y_{T}\right)\right)_{2}\right) \leqq C$ and therefore, due to Lemma 3.1(v), we get

$$
\#\left\{x \in \hat{X}_{T} \cap Q_{T}^{v}\left(y_{T}\right): \#\left(\mathcal{N}(x) \cap \hat{X}_{T}\right)<\#\left(\mathcal{N}(x) \cap X_{T}\right)\right\} \leqq C .
$$

In a similar fashion, if $x \in \hat{X}_{T} \cap\left(Q_{T+32}^{v}\left(y_{T}\right) \backslash Q_{T}^{v}\left(y_{T}\right)\right)$ such that $\#\left(\mathcal{N}(x) \cap \hat{X}_{T}\right)<$ 6 , then necessarily $x \in\left(A_{T}\right)_{1} \cap Q_{T+32}^{v}\left(y_{T}\right)$. Thus, again by Lemma 3.1(v), only a bounded number of atoms in $Q_{T+32}^{\nu}\left(y_{T}\right) \backslash Q_{T}^{\nu}\left(y_{T}\right)$ independently of $T$ has less than six neighbors. This along with (7.12) and (2.3) yields (7.11). by

Let us now define $\tilde{X}_{T}$. We recall the notation in (2.4) and define $\tilde{X}_{T}=\tilde{X}_{T}^{+} \cup \tilde{X}_{T}^{-}$

$$
\tilde{X}_{T}^{+}=\left(\hat{X}_{T}^{+}+e^{i \theta^{+}}\left(\tau^{+}-\tau_{T}^{+}\right)\right), \quad \tilde{X}_{T}^{-}=\left(\hat{X}_{T}^{-}+e^{i \theta^{-}}\left(\tau^{-}-\tau_{T}^{-}\right)\right) .
$$

For convenience, we denote the atoms of $\hat{X}_{T}$ by $\left\{x_{T}^{j}\right\}_{j}$ and the corresponding atoms of $\tilde{X}_{T}$ by $\left\{\tilde{x}_{T}^{j}\right\}_{j}$, that is, $\tilde{x}_{T}^{j}=x_{T}^{j}+e^{i \theta^{ \pm}}\left(\tau^{ \pm}-\tau_{T}^{ \pm}\right)$if $x_{T}^{j} \in \hat{X}_{T}^{ \pm}$. By (7.10) and the choice of $\hat{X}_{T}$, it is obvious that $\tilde{X}_{T}^{ \pm} \subset \mathscr{L}\left(z^{ \pm}\right)$and $\tilde{X}_{T}^{ \pm}=\mathscr{L}\left(z^{ \pm}\right)$on $\partial_{1}^{ \pm} Q_{T+22}^{v}\left(y_{T}\right)$ for $T$ large enough. Here, the extension $\hat{X}_{T}=\mathscr{L}\left(z_{T}^{ \pm}\right)$on $\left\{x: \pm\left\langle v, x-y_{T}\right\rangle \geqq 2\right\} \cap$ $\left(Q_{T+34}^{v}\left(y_{T}\right) \backslash\left(Q_{T+10}^{v}\left(y_{T}\right) \cup A_{T}\right)\right)$ is crucial in order to ensure that these boundary conditions hold for $\tilde{X}_{T}$. (The value 2 is for definiteness only. Every value less than 5 works, provided $T$ is sufficiently large.) To show (7.9), we prove

$$
E_{1}\left(\tilde{X}_{T}, Q_{T+22}^{v}\left(y_{T}\right)\right) \leqq E_{1}\left(\hat{X}_{T}, Q_{T+32}^{v}\left(y_{T}\right)\right) .
$$

Then, the result follows from (7.11). To this end, we need to check the following for large $T$ :

$$
\text { (i) }\left|x_{T}^{j}-x_{T}^{k}\right|=1 \Longrightarrow\left|\tilde{x}_{T}^{j}-\tilde{x}_{T}^{k}\right|=1, \quad \text { and } \quad \text { (ii) }\left|\tilde{x}_{T}^{j}-\tilde{x}_{T}^{k}\right| \geqq 1 \text { for all } j, k, j \neq k \text {. }
$$

In fact, due to (7.13)(ii), $\tilde{X}_{T}$ is a configuration with finite energy. Moreover, (7.13)(i) shows that $x_{T}^{k} \in \mathcal{N}\left(x_{T}^{j}\right)$ implies $\tilde{x}_{T}^{k} \in \mathcal{N}\left(\tilde{x}_{T}^{j}\right)$, and therefore the energy can only decrease, see (2.3).

Let us finally check (7.13). If both atoms are in $\hat{X}_{T}^{-}$or $\hat{X}_{T}^{+}$, then it is clear by the definition of $\tilde{X}_{T}$ that $x_{T}^{j}-x_{T}^{k}=\tilde{x}_{T}^{j}-\tilde{x}_{T}^{k}$, which gives (i) and (ii) due to (7.7) and (7.11). Otherwise, if $x_{T}^{j} \in \hat{X}_{T}^{-}$and $x_{T}^{k} \in \hat{X}_{T}^{+}$or vice versa, (i) follows from Lemma 7.4, whereas (ii) follows from Lemma 7.4(i), (7.7) and (7.11).

To conclude the proof of Lemma 7.1, it remains to give the proofs of Lemmas 7.3 and 7.4.

Proof of Lemma 7.3. Let $z_{T}^{ \pm}=\left(\theta_{T}^{ \pm}, \tau_{T}^{ \pm}, 1\right) \in \mathcal{Z}$ and $v \in \mathbb{S}^{1}$ be given as in the statement.

Step 1: Rotation to boundary conditions with fixed rotation angles. Choose $\tilde{y}_{T} \in \mathbb{R}^{2}$ and $\tilde{X}_{T} \subset \mathbb{R}^{2}$ satisfying $\tilde{X}_{T}=\mathscr{L}\left(z_{T}^{ \pm}\right)$on $\partial_{1}^{ \pm} Q_{T}^{v}\left(\tilde{y}_{T}\right)$ such that

$$
E_{1}\left(\tilde{X}_{T}, Q_{T}^{v}\left(\tilde{y}_{T}\right)\right) \leqq \inf \left\{E_{1}\left(X_{T}, Q_{T}^{v}\left(y_{T}\right)\right): y_{T} \in \mathbb{R}^{2}, X_{T}\right.
$$




$$
\left.=\mathscr{L}\left(z_{T}^{ \pm}\right) \text {on } \partial_{1}^{ \pm} Q_{T}^{v}\left(y_{T}\right)\right\}+1 / T \text {. }
$$

We define $X_{T}^{\mathrm{rot}}:=e^{i\left(\theta^{+}-\theta_{T}^{+}\right)} \tilde{X}_{T}, v_{T}:=e^{i\left(\theta^{+}-\theta_{T}^{+}\right)} \nu, y_{T}^{\text {rot }}:=e^{i\left(\theta^{+}-\theta_{T}^{+}\right)} \tilde{y}_{T}$, and $\hat{z}^{ \pm}:=\left(\theta^{ \pm}, \tau_{T}^{ \pm}, 1\right)$. Then, by Lemma 3.1(i) and $\theta_{T}^{+}-\theta_{T}^{-}=\theta^{+}-\theta^{-}$for all $T$, there holds $X_{T}^{\text {rot }}=\mathscr{L}\left(\hat{z}_{T}^{ \pm}\right)$on $\partial_{1}^{ \pm} Q_{T}^{\nu_{T}}\left(y_{T}^{\text {rot }}\right)$ and

$$
\begin{aligned}
& E_{1}\left(\tilde{X}_{T}, Q_{T}^{\nu}\left(\tilde{y}_{T}\right)\right)=E_{1}\left(X_{T}^{\mathrm{rot}}, Q_{T}^{\nu_{T}}\left(y_{T}^{\mathrm{rot}}\right)\right) \\
& \quad \geqq \inf \left\{E_{1}\left(X_{T}, Q_{T}^{\nu_{T}}\left(y_{T}\right)\right): y_{T} \in \mathbb{R}^{2}, X_{T}=\mathscr{L}\left(\hat{z}_{T}^{ \pm}\right) \text {on } \partial_{1}^{ \pm} Q_{T}^{\nu_{T}}\left(y_{T}\right)\right\}
\end{aligned}
$$

for all $T>0$. Therefore, in view of (7.14), to show the statement it suffices to prove

$$
\begin{aligned}
& \liminf _{T \rightarrow+\infty} \frac{1}{T} \inf \left\{E_{1}\left(X_{T}, Q_{T}^{\nu_{T}}\left(y_{T}\right)\right): y_{T} \in \mathbb{R}^{2}, X_{T}=\mathscr{L}\left(\hat{z}_{T}^{ \pm}\right) \text {on } \partial_{1}^{ \pm} Q_{T}^{\nu_{T}}\left(y_{T}\right)\right\} \\
& \quad \geqq \liminf _{T \rightarrow+\infty} \frac{1}{T} \inf \left\{E_{1}\left(X_{T}, Q_{T}^{\nu}\left(y_{T}\right)\right): y_{T} \in \mathbb{R}^{2}, X_{T}=\mathscr{L}\left(\hat{z}_{T}^{ \pm}\right) \text {on } \partial_{1}^{ \pm} Q_{T}^{v}\left(y_{T}\right)\right\} .
\end{aligned}
$$

Note that the difference of the two formulas lies only in the fact that $v$ is replaced by $v_{T}$, where $v_{T} \rightarrow v$ as $T \rightarrow+\infty$.

Step 2: Proof of (7.15). Fix $\delta>0$ and let $T>0$ be sufficiently large such that $\left|\nu_{T}-v\right|<\delta$. We choose $\tilde{y}_{T} \in \mathbb{R}^{2}$ and $\tilde{X}_{T} \subset \mathbb{R}^{2}$ satisfying $\tilde{X}_{T}=\mathscr{L}\left(\hat{z}_{T}^{ \pm}\right)$on $\partial_{1}^{ \pm} Q_{T}^{\nu_{T}}\left(\tilde{y}_{T}\right)$ such that

$$
\begin{aligned}
E_{1}\left(\tilde{X}_{T}, Q_{T}^{\nu_{T}}\left(\tilde{y}_{T}\right)\right) \leqq \inf \{ & E_{1}\left(X_{T}, Q_{T}^{v_{T}}\left(y_{T}\right)\right): y_{T} \in \mathbb{R}^{2}, \\
& \left.X_{T}=\mathscr{L}\left(\hat{z}_{T}^{ \pm}\right) \text {on } \partial_{1}^{ \pm} Q_{T}^{\nu_{T}}\left(y_{T}\right)\right\}+\delta .
\end{aligned}
$$

Recall (2.4) and (2.5). We set $T_{\delta}=(1+2 \delta) T$ and define

$$
\begin{aligned}
A_{T}^{\delta}= & \left(\left[\tilde{y}_{T}-\frac{T}{2} v_{T}^{\perp} ; \tilde{y}_{T}-\frac{T_{\delta}}{2} v^{\perp}\right] \cup\left[\tilde{y}_{T}+\frac{T}{2} v_{T}^{\perp} ; \tilde{y}_{T}+\frac{T_{\delta}}{2} v^{\perp}\right]\right)_{\kappa T \delta} \\
& \backslash\left(\partial_{1}^{+} Q_{T_{\delta}}^{v}\left(\tilde{y}_{T}\right) \cup \partial_{1}^{-} Q_{T_{\delta}}^{\nu}\left(\tilde{y}_{T}\right)\right),
\end{aligned}
$$

where $\kappa>1$ is chosen sufficiently large later. We define the configuration $\hat{X}_{T} \subset \mathbb{R}^{2}$ by

$$
\hat{X}_{T}= \begin{cases}\tilde{X}_{T} & \text { in } Q_{T}^{\nu_{T}}\left(\tilde{y}_{T}\right), \\ \varnothing & \text { in } A_{T}^{\delta} \backslash Q_{T}^{\nu_{T}}\left(\tilde{y}_{T}\right), \\ \mathscr{L}\left(\hat{z}_{T}^{ \pm}\right) & \text {in }\left\{x: \pm\left\langle v,\left(x-\tilde{y}_{T}\right)\right\rangle \geqq 5\right\} \backslash\left(A_{T}^{\delta} \cup Q_{T}^{\nu_{T}}\left(\tilde{y}_{T}\right)\right) .\end{cases}
$$

Here, $\kappa>1$ is chosen large enough (independently of $T$ ) such that $|x-y| \geqq 1$ for all $x, y \in \hat{X}_{T}, x \neq y$. In principle, $|x-y|<1$ may occur for points $x \in \tilde{X}_{T} \cap Q_{T}^{v_{T}}\left(\tilde{y}_{T}\right)$ and $y \in \mathbb{R}^{2} \backslash Q_{T}^{\nu_{T}}\left(\tilde{y}_{T}\right)$ if $x \in Q_{T}^{\nu_{T}}\left(\tilde{y}_{T}\right) \backslash Q_{T-2}^{\nu_{T}}\left(\tilde{y}_{T}\right), \pm\left\langle\nu_{T},\left(x-\tilde{y}_{T}\right)\right\rangle \geqq-5$ and $\pm\left\langle\nu,\left(y-\tilde{y}_{T}\right)\right\rangle \leqq-5$, but for $\kappa$ big enough such pairs of points are contained in $A_{T}^{\delta}$. 
We note that $\partial_{1}^{ \pm} Q_{T_{\delta}}^{v}\left(\tilde{y}_{T}\right) \cap Q_{T}^{v}\left(\tilde{y}_{T}\right)=\emptyset$ for $T$ large enough since $v_{T} \rightarrow v$ as $T \rightarrow+\infty$. Thus, by construction we get $\hat{X}_{T}=\mathscr{L}\left(\hat{z}_{T}^{ \pm}\right)$on $\partial_{1}^{ \pm} Q_{T_{\delta}}^{v}\left(\tilde{y}_{T}\right)$ for $T$ sufficiently large. Therefore, we obtain

$$
\begin{aligned}
& \inf \left\{E_{1}\left(X_{T}, Q_{T_{\delta}}^{v}\left(y_{T}\right)\right): y_{T} \in \mathbb{R}^{2}, X_{T}=\mathscr{L}\left(\hat{z}_{T}^{ \pm}\right) \text {on } \partial_{1}^{ \pm} Q_{T_{\delta}}^{v}\left(y_{T}\right)\right\} \\
& \quad \leqq E_{1}\left(\hat{X}_{T}, Q_{T_{\delta}}^{v}\left(\tilde{y}_{T}\right)\right) .
\end{aligned}
$$

We claim that

$$
E_{1}\left(\hat{X}_{T}, Q_{T_{\delta}}^{v}\left(\tilde{y}_{T}\right)\right) \leqq E_{1}\left(\tilde{X}_{T}, Q_{T}^{v_{T}}\left(\tilde{y}_{T}\right)\right)+C \kappa \delta T
$$

for a universal $C>0$. We defer the proof of this estimate to Step 3 below and conclude the proof of (7.15). Dividing (7.19) by $T_{\delta}$ and letting $T \rightarrow+\infty$, we derive

$$
\liminf _{T \rightarrow+\infty} \frac{1}{T_{\delta}} E_{1}\left(\hat{X}_{T}, Q_{T_{\delta}}^{v}\left(\tilde{y}_{T}\right)\right) \leqq \liminf _{T \rightarrow+\infty} \frac{1}{T} E_{1}\left(\tilde{X}_{T}, Q_{T}^{\nu_{T}}\left(\tilde{y}_{T}\right)\right)+C \kappa \delta .
$$

This along with (7.16) and (7.18), and the fact that $\delta>0$ was arbitrary shows (7.15). It thus remains to prove (7.19).

Step 3: Proof of (7.19). We divide the proof into the two estimates

$$
\begin{aligned}
& E_{1}\left(\hat{X}_{T}, Q_{T}^{\nu_{T}}\left(\tilde{y}_{T}\right)\right) \leqq E_{1}\left(\tilde{X}_{T}, Q_{T}^{\nu_{T}}\left(\tilde{y}_{T}\right)\right)+C \kappa \delta T, \text { and } \\
& E_{1}\left(\hat{X}_{T}, Q_{T_{\delta}}^{v_{1}}\left(\tilde{y}_{T}\right) \backslash Q_{T}^{\nu_{T}}\left(\tilde{y}_{T}\right)\right) \leqq C \kappa \delta T,
\end{aligned}
$$

for a universal $C>0$. Clearly, (7.20)-(7.21) and Lemma 3.1(iv) imply (7.19). We first prove (7.20). Recall by (7.17) and the boundary values of $\tilde{X}_{T}$ that $\hat{X}_{T}=\tilde{X}_{T}$ in $\overline{Q_{T+2}^{\nu_{T}}\left(\tilde{y}_{T}\right)} \backslash\left(A_{T}^{\delta} \backslash Q_{T}^{\nu_{T}}\left(\tilde{y}_{T}\right)\right)$. Thus, $x \in Q_{T}^{\nu_{T}}\left(\tilde{y}_{T}\right)$ can have less neighbors in $\hat{X}_{T}$ than in $\tilde{X}_{T}$ only if $x \in\left(A_{T}^{\delta}\right)_{1} \cap\left(Q_{T}^{v_{T}}\left(\tilde{y}_{T}\right) \backslash Q_{T-2}^{\nu_{T}}\left(\tilde{y}_{T}\right)\right)$. As $\operatorname{diam}\left(A_{T}^{\delta}\right) \leqq C \kappa \delta T$ and therefore $\mathcal{L}^{2}\left(\left(\left(A_{T}^{\delta}\right)_{1} \cap\left(Q_{T}^{\nu_{T}}\left(\tilde{y}_{T}\right) \backslash Q_{T-2}^{\nu_{T}}\left(\tilde{y}_{T}\right)\right)\right)_{1}\right) \leqq C \kappa \delta T$, this implies by Lemma 3.1(v) that a number of atoms $x \in Q_{T}^{\nu_{T}}\left(\tilde{y}_{T}\right)$ bounded by $C \kappa \delta T$ have less neighbors in $\hat{X}_{T}$ than in $\tilde{X}_{T}$. This shows (7.20) by (2.3). To see (7.21), again due to (7.17), all atoms $x \in \hat{X}_{T} \cap\left(Q_{T_{\delta}}^{v}\left(\tilde{y}_{T}\right) \backslash\left(Q_{T}^{\nu_{T}}\left(\tilde{y}_{T}\right) \cup\left(A_{T}^{\delta}\right)_{1}\right)\right.$ have six neighbors. Hence, their energy contribution is zero. As $\hat{X}_{T}=\emptyset$ in $A_{T}^{\delta} \backslash Q_{T}^{\nu_{T}}\left(\tilde{y}_{T}\right)$ and $\mathcal{L}^{2}\left(\left(\left(A_{T}^{\delta}\right)_{1} \backslash A_{T}^{\delta}\right)_{1}\right) \leqq C \kappa \delta T$, this implies, as before, that

$$
\#\left(\hat{X}_{T} \cap\left(\left(A_{T}^{\delta}\right)_{1} \cap Q_{T_{\delta}}^{v_{y}}\left(\tilde{y}_{T}\right)\right) \backslash Q_{T}^{\nu_{T}}\left(\tilde{y}_{T}\right)\right) \leqq C \mathcal{L}^{2}\left(\left(\left(A_{T}^{\delta}\right)_{1} \backslash A_{T}^{\delta}\right)_{1}\right) \leqq C \kappa \delta T .
$$

Again, in view of (2.3), this implies (7.21), and concludes the proof.

Proof of Lemma 7.4. Suppose first that $y \in P_{\theta^{+}, \theta^{-}}$with $P_{\theta^{+}, \theta^{-}}$defined in (7.6). Then (i) follows from $x_{n}^{+} \rightarrow x, y_{n}^{-} \rightarrow y$, and the observation that there are only finitely many pairs $(x, y) \in \mathscr{L}\left(z^{+}\right) \times\left(P_{\theta^{+}, \theta^{-}} \cap \mathscr{L}\left(z^{-}\right)\right)$with $|x-y|<1$. The same argument applies to show that (ii) holds true for all pairs $(x, y) \in\left(\left(P_{\theta^{+}, \theta^{-}}\right)_{3} \cap\right.$ $\left.\mathscr{L}\left(z^{+}\right)\right) \times\left(P_{\theta^{+}, \theta^{-}} \cap \mathscr{L}\left(z^{-}\right)\right)$for large $n$. Choosing $n$ so big that $\left|\tau_{n}^{ \pm}-\tau^{ \pm}\right|<1$ also gives (ii) for all $(x, y) \in \mathscr{L}\left(z^{+}\right) \times\left(P_{\theta^{+}, \theta^{-}} \cap \mathscr{L}\left(z^{-}\right)\right)$.

Now, consider a general $y \in \mathscr{L}\left(z^{-}\right)$. One finds $v \in e^{i \theta^{+}} \mathscr{L} \cap e^{i \theta^{-}} \mathscr{L}$ such that $y-v \in P_{\theta^{+}, \theta^{-}}$. The assertion then follows by applying the special case 
described above to $x-v$ and $y-v$, and by observing that $(x-v)_{n}^{+}=x_{n}^{+}-v$ and $(y-v)_{n}^{-}=y_{n}^{-}-v$. Finally, the implication $\left|x_{n}^{+}-y_{n}^{-}\right|=1 \Rightarrow|x-y|=1$ follows from (i) and (ii) by contraposition.

\subsection{Well Definedness and Properties of the Energy Density $\varphi$}

This final subsection is devoted to the proofs of Proposition 7.2 and Theorem 2.5. Our proofs in this subsection follow standard strategies. Due to the discrete character of our model, however, careful constructions are needed. As a preliminary step, we show that in (7.1) the sequence $T \rightarrow+\infty$ can be chosen independently of the centers of the cells.

Proposition 7.5. For each $z^{+}, z^{-} \in \mathcal{Z}$ and $v \in \mathbb{S}^{1}$ there exists a sequence $\left\{T_{j}\right\}_{j}$ such that $T_{j} \rightarrow+\infty$ as $j \rightarrow+\infty$ and for all $\left\{y_{j}\right\}_{j} \subset \mathbb{R}^{2}$ it holds that

$$
\frac{1}{T_{j}} \min \left\{E_{1}\left(X, Q_{T_{j}}^{v}\left(y_{j}\right)\right): X=\mathscr{L}\left(z^{ \pm}\right) \text {on } \partial_{1}^{ \pm} Q_{T_{j}}^{v}\left(y_{j}\right)\right\} \leqq \bar{\varphi}\left(z^{+}, z^{-}, v\right)+\eta_{j},
$$

where $\left\{\eta_{j}\right\}_{j} \subset(0,+\infty)$ is a null sequence which depends on $z^{ \pm}$and $v$, but is independent of $\left\{y_{j}\right\}_{j}$.

Proof. First, if $z^{+}=\mathbf{0}$ or $z^{-}=\mathbf{0}$, the statement follows from Lemma 6.1(i) and the definition of $\bar{\varphi}$ in (7.1) for any sequence $\left\{T_{j}\right\}_{j}$. Now consider $z^{ \pm}=\left(\theta^{ \pm}, \tau^{ \pm}, 1\right)$. If $\theta^{+}-\theta^{-} \notin \mathcal{G}_{\mathbb{A}}$, the statement follows from Lemma 6.1(ii), (6.1), and Lemma 6.2 for any sequence $\left\{T_{j}\right\}_{j}$. Therefore, it remains to treat the case $\theta^{+}-\theta^{-} \in \mathcal{G}_{\mathbb{A}}$.

Consider a sequence $S_{j} \rightarrow+\infty,\left\{x_{j}\right\}_{j} \subset \mathbb{R}^{2}$, and configurations $\left\{X_{j}\right\}_{j} \subset \mathbb{R}^{2}$ satisfying $X_{j}=\mathscr{L}\left(z^{ \pm}\right)$on $\partial_{1}^{ \pm} Q_{S_{j}}^{v}\left(x_{j}\right)$ such that

$$
\bar{\varphi}\left(z^{+}, z^{-}, v\right)=\lim _{j \rightarrow+\infty} \frac{1}{S_{j}} E_{1}\left(X_{j}, Q_{S_{j}}^{v}\left(x_{j}\right)\right) .
$$

By Lemma 5.1 it is not restrictive to assume that $X_{j} \subset \mathscr{L}\left(z^{ \pm}\right)$for all $j \in \mathbb{N}$. Our goal is to find a sequence $l_{j} \rightarrow 1$ such that for all $\left\{y_{j}\right\}_{j}$ there are configurations $\left\{\tilde{X}_{j}\right\}_{j} \subset \mathbb{R}^{2}$ satisfying $\tilde{X}_{j}=\mathscr{L}\left(z^{ \pm}\right)$on $\partial_{1}^{ \pm} Q_{l_{j} S_{j}}^{v}\left(y_{j}\right)$ such that

$$
E_{1}\left(\tilde{X}_{j}, Q_{l_{j} S_{j}}^{v}\left(y_{j}\right)\right) \leqq E_{1}\left(X_{j}, Q_{S_{j}}^{v}\left(x_{j}\right)\right)+C
$$

for a constant $C>0$ only depending on $z^{ \pm}$and $\nu$. Once this is achieved, we obtain the statement as follows: we introduce the sequence $T_{j}:=l_{j} S_{j}$, divide (7.23) by $T_{j}$, and use (7.22) to get

$$
\begin{aligned}
& \frac{1}{T_{j}} \min \left\{E_{1}\left(X, Q_{T_{j}}^{v}\left(y_{j}\right)\right): X=\mathscr{L}\left(z^{ \pm}\right) \text {on } \partial_{1}^{ \pm} Q_{T_{j}}^{v}\left(y_{j}\right)\right\} \\
& \quad \leqq \frac{1}{T_{j}} E_{1}\left(\tilde{X}_{j}, Q_{l_{j} S_{j}}^{v}\left(y_{j}\right)\right) \\
& \quad \leqq \frac{1}{l_{j} S_{j}} E_{1}\left(X_{j}, Q_{S_{j}}^{v}\left(x_{j}\right)\right)+\frac{C}{T_{j}}
\end{aligned}
$$




$$
\leqq \bar{\varphi}\left(z^{+}, z^{-}, v\right)+\eta_{j},
$$

where $\left\{\eta_{j}\right\}_{j}$ is a null sequence only depending on $z^{+}, z^{-}, v$, and $\left\{T_{j}\right\}_{j}$, but independent of the centers $\left\{y_{j}\right\}_{j}$.

Consider any sequence of centers $\left\{y_{j}\right\}_{j}$. We now construct $\tilde{X}_{j}$ and confirm (7.23). We choose $\bar{y}_{j} \in\left(\mathscr{L}\left(z^{+}\right) \cap \mathscr{L}\left(z^{-}\right)\right)+x_{j}$ such that $\left|y_{j}-\bar{y}_{j}\right| \leqq \kappa$, where $\kappa:=|a|+|b|+5$ only depends on the spanning vectors $a, b$ in (7.5), but is independent of $j$. Let $l_{j}:=1+4 \kappa / S_{j}$. We set

$$
A_{j}=\left(\left[\bar{y}_{j}-\frac{S_{j}}{2} v^{\perp} ; y_{j}-\frac{l_{j} S_{j}}{2} v^{\perp}\right]\right)_{4 \kappa} \cup\left(\left[\bar{y}_{j}+\frac{S_{j}}{2} v^{\perp} ; y_{j}+\frac{l_{j} S_{j}}{2} v^{\perp}\right]\right)_{4 \kappa} .
$$

Note that $\partial_{1}^{ \pm} Q_{l_{j} S_{j}}^{v}\left(y_{j}\right) \cap Q_{S_{j}}^{v}\left(\bar{y}_{j}\right)=\emptyset$ since $S_{j} l_{j}-S_{j}=4 \kappa,\left|y_{j}-\bar{y}_{j}\right| \leqq \kappa$, and $\kappa \geqq 5$. We define $\tilde{X}_{j} \subset \mathbb{R}^{2}$ by

$$
\tilde{X}_{j}= \begin{cases}X_{j}+\bar{y}_{j}-x_{j} & \text { in } Q_{S_{j}}^{v}\left(\bar{y}_{j}\right) \backslash A_{j}, \\ \varnothing & \text { in } A_{j} \backslash\left(\partial_{1}^{+} Q_{l_{j} S_{j}}^{v}\left(y_{j}\right) \cup \partial_{1}^{-} Q_{l_{j} S_{j}}^{v}\left(y_{j}\right)\right), \\ \mathscr{L}\left(z^{ \pm}\right) & \text {in }\left(\left\{ \pm\left\langle\nu, x-y_{j}\right\rangle \geqq 5\right\} \backslash\left(A_{j} \cup Q_{S_{j}}^{v}\left(\bar{y}_{j}\right)\right)\right) \cup \partial_{1}^{ \pm} Q_{l_{j} S_{j}}^{v}\left(y_{j}\right) .\end{cases}
$$

By definition, $\tilde{X}_{j}$ attains the correct boundary conditions, and therefore it remains to check (7.23). First, as $x_{j}-\bar{y}_{j} \in \mathscr{L}\left(z^{+}\right) \cap \mathscr{L}\left(z^{-}\right)$and $X_{j}=\mathscr{L}\left(z^{ \pm}\right)$on $\partial_{1}^{ \pm} Q_{S_{j}}^{v}\left(x_{j}\right)$, we observe that $\tilde{X}_{j}=\mathscr{L}\left(z^{ \pm}\right)$on $\left(\partial_{1}^{ \pm} Q_{S_{j}}^{v}\left(\bar{y}_{j}\right) \cap Q_{S_{j}}^{v}\left(\bar{y}_{j}\right)\right) \backslash A_{j}$. This along with the definition of $A_{j}$ implies $|x-y| \geqq 1$ for all $x, y \in \tilde{X}_{j}, x \neq y$, and thus $E_{1}\left(\tilde{X}_{j}, Q_{l_{j} S_{j}}^{v}\left(y_{j}\right)\right)<+\infty$. Moreover, by Lemma 3.1(i) we obtain

$$
E_{1}\left(\tilde{X}_{j}, Q_{S_{j}}^{v}\left(\bar{y}_{j}\right)\right) \leqq E_{1}\left(X_{j}, Q_{S_{j}}^{v}\left(x_{j}\right)\right)+C .
$$

Here, the extra term $C>0$ is due the fact that we take into account the interactions of points $x \in \tilde{X}_{j} \cap Q_{S_{j}}^{v}\left(\bar{y}_{j}\right) \cap\left(A_{j}\right)_{1}$. Since $\mathcal{L}^{2}\left(\left(A_{j}\right)_{2}\right) \leqq C_{\kappa}$ for $C_{\kappa}$ depending only $\kappa$ and $E_{1}\left(\tilde{X}_{j}\right)<+\infty$, by Lemma 3.1(v), the cardinality of these points can be controlled by $C_{\kappa}$. Then, by (2.3) we indeed get (7.24). Additionally, it holds that

$$
E_{1}\left(\tilde{X}_{j}, Q_{l_{j} S_{j}}^{v}\left(y_{j}\right) \backslash Q_{S_{j}}^{v}\left(\bar{y}_{j}\right)\right) \leqq C,
$$

where $C$ again only depends on $\kappa$. In fact, all points $x \in \tilde{X}_{j} \cap\left(Q_{l_{j} S_{j}}^{v}\left(y_{j}\right) \backslash Q_{S_{j}}^{v}\left(\bar{y}_{j}\right)\right)$ with $\operatorname{dist}\left(x, A_{j}\right)>1$ satisfy $\# \mathcal{N}(x)=6$ and therefore they do not contribute to the energy. Again due to Lemma 3.1(v), the cardinality of $x \in \tilde{X}_{j}$ with $\operatorname{dist}\left(x, A_{j}\right) \leqq 1$ can be estimated by $C_{\kappa}$. This gives (7.25). Now, (7.24)-(7.25) along with Lemma 3.1(iv) imply (7.23). This concludes the proof.

Proof of Proposition 7.2. We first show that, once (7.3) has been established, the result in Proposition 2.2 follows. Indeed, given $x_{0} \in \mathbb{R}^{2}$ and $\rho>0$, estimate (2.16) readily follows from (7.3) for the sequence of centers $y_{T}=(T / \rho) x_{0}$ and a scaling argument, see Proposition 3.1(ii) for $\varepsilon=\rho / T, \lambda=T / \rho$, and $A=Q_{\rho}^{v}\left(x_{0}\right)$. 
It remains to prove (7.3). Let $z^{ \pm} \in \mathcal{Z}, v \in \mathbb{S}^{1}$, and a sequence $\left\{y_{T}\right\}_{T} \subset \mathbb{R}^{2}$ be given. In view of the definition of $\bar{\varphi}$, see (7.1), it suffices to show that

$\limsup _{T \rightarrow+\infty} \frac{1}{T} \min \left\{E_{1}\left(X_{T}, Q_{T}^{v}\left(y_{T}\right)\right): X_{T}=\mathscr{L}\left(z^{ \pm}\right)\right.$on $\left.\partial_{1}^{ \pm} Q_{T}^{v}\left(y_{T}\right)\right\} \leqq \bar{\varphi}\left(z^{+}, z^{-}, v\right)$.

Step 1: Comparison via construction. Consider $1 \ll S \ll T$. Without restriction, we can assume that $S \in\left\{T_{j}\right\}_{j}$, where $\left\{T_{j}\right\}_{j}$ is the sequence identified in Proposition 7.5. For simplicity, if $S=T_{j}$, we will write $\eta_{S}$ instead of $\eta_{T_{j}}$ for the null sequence given by Proposition 7.5. Define $N_{S, T}:=\lfloor T / S\rfloor$. For $j \in\left\{1, \ldots, N_{S, T}\right\}$ we set $x_{j}=y_{T}+(-T / 2-S / 2+j S) v^{\perp}$. We choose $X_{j} \subset \mathbb{R}^{2}$ such that $X_{j}=\mathscr{L}\left(z^{ \pm}\right)$ on $\partial_{1}^{ \pm} Q_{S}^{v}\left(x_{j}\right)$ and

$$
\begin{aligned}
E_{1}\left(X_{j}, Q_{S}^{v}\left(x_{j}\right)\right) & =\min \left\{E_{1}\left(X, Q_{S}^{v}\left(x_{j}\right)\right): X=\mathscr{L}\left(z^{ \pm}\right) \text {on } \partial_{1}^{ \pm} Q_{S}^{v}\left(x_{j}\right)\right\} \\
& \leqq S\left(\bar{\varphi}\left(z^{+}, z^{-}, \nu\right)+\eta_{S}\right),
\end{aligned}
$$

where the inequality follows from Proposition 7.5. For $j=1, \ldots, N_{S, T}$, we introduce the set $A_{j}=Q_{10}^{v}\left(x_{j}+(S / 2) v^{\perp}\right) \cup Q_{10}^{v}\left(x_{j}-(S / 2) v^{\perp}\right)$ and let $X_{T}$ be defined by

$$
X_{T}= \begin{cases}X_{j} & \text { in } Q_{S}^{v}\left(x_{j}\right) \backslash A_{j}, j \in\left\{1, \ldots, N_{S, T}\right\}, \\ \varnothing & \text { in }\left\{x:\left|\left\langle v, x-y_{T}\right\rangle\right|<5\right\} \backslash Q^{*}, \\ \mathscr{L}\left(z^{ \pm}\right) & \text {in }\left\{x: \pm\left\langle v, x-y_{T}\right\rangle \geqq 5\right\} \backslash Q^{*},\end{cases}
$$

where for brevity we have set $Q^{*}:=\bigcup_{j=1}^{N_{S, T}}\left(Q_{S}^{v}\left(x_{j}\right) \backslash A_{j}\right)$. Note that $X_{T}=\mathscr{L}\left(z^{ \pm}\right)$ on $\partial_{1}^{ \pm} Q_{T}^{v}\left(y_{T}\right)$. For an illustration of the construction, we refer to Fig. 13. We will show that

$$
E_{1}\left(X_{T}, Q_{T}^{v}\left(y_{T}\right)\right) \leqq\lfloor T / S\rfloor S\left(\bar{\varphi}\left(z^{+}, z^{-}, v\right)+\eta_{S}\right)+C T / S+C S
$$

for a universal constant $C>0$. Once this is achieved, we divide by $T$, take first the lim sup as $T \rightarrow+\infty$, and then the limit as $S \rightarrow+\infty$ (with $S$ chosen from the sequence $\left\{T_{j}\right\}_{j}$ given by Proposition 7.5). As $\eta_{S} \rightarrow 0$, this yields (7.26) and thus the statement of the proposition.

Step 2: Proof of (7.28). It remains to prove (7.28). First, by construction, the definition of $A_{j}$, and the boundary values of the configurations $X_{j}$, we get $|x-y| \geqq$ 1 for all $x, y \in X_{T}, x \neq y$, and therefore $E\left(X_{T}\right)<+\infty$. By Lemma 3.1(iv) and (7.27) it holds that

$$
\begin{aligned}
& E_{1}\left(X_{T}, Q_{T}^{v}\left(y_{T}\right)\right)=\sum_{j=1}^{N_{S, T}} E\left(X_{T}, Q_{S}^{v}\left(x_{j}\right)\right)+E\left(X_{T}, Q_{T}^{v}\left(y_{T}\right) \backslash \bigcup_{j=1}^{N_{S, T}} Q_{S}^{v}\left(x_{j}\right)\right) \\
& \quad \leqq\lfloor T / S\rfloor\left(S\left(\bar{\varphi}\left(z^{+}, z^{-}, v\right)+\eta_{S}\right)+C\right)+E\left(X_{T}, Q_{T}^{v}\left(y_{T}\right) \backslash \bigcup_{j=1}^{N_{S, T}} Q_{S}^{v}\left(x_{j}\right)\right) .
\end{aligned}
$$



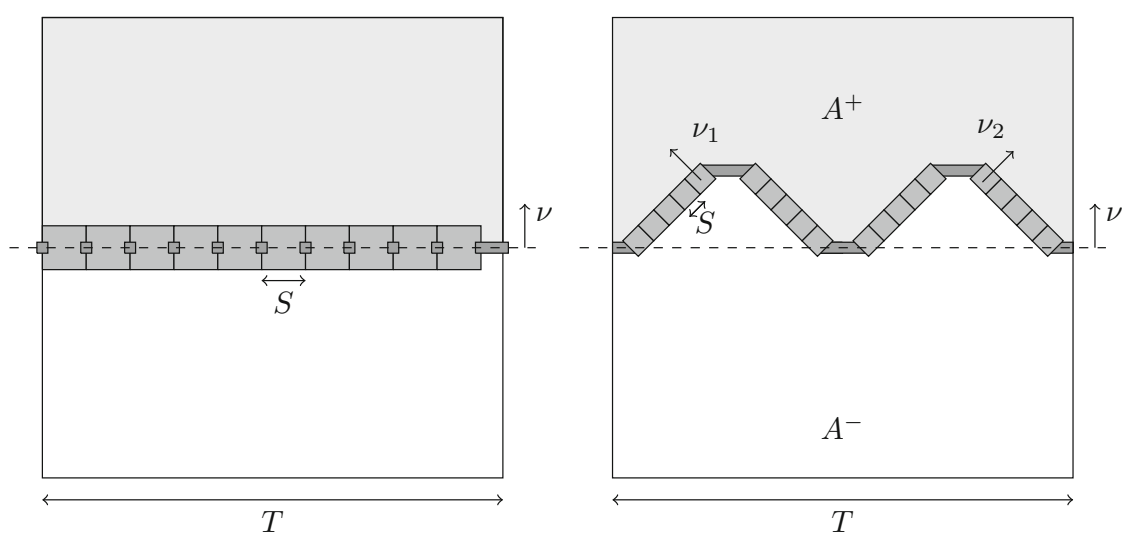

Fig. 13. Illustration of the construction for the existence of the limit on the left as well as the convexity in the third variable on the right. On the white region $X_{T}=\mathscr{L}\left(z^{-}\right)$, on the light gray region $X_{T}=\mathscr{L}\left(z^{+}\right)$, and on the dark gray region $X_{T}=\emptyset$. The dark gray cubes, that are cut out in order to ensure that $X_{T}$ has finite energy, are illustrated on the left, but they are also present in the construction on the right. In the gray cubes, we set $X_{T}$ equal to the minimizer with boundary conditions $\mathscr{L}\left(z^{ \pm}\right)$. For illustration purposes, we suppose that $w=0$ in (7.34)

Here, the addend $C$ in the brackets is due to the fact that there may be $x \in X_{T} \cap$ $Q_{S}^{v}\left(x_{j}\right)$ with more neighbors in $X_{j}$ than in $X_{T}$. This, however, can only occur for atoms in $x \in Q_{S}^{v}\left(x_{j}\right)$ such that $x \in\left(\partial Q_{S}^{v}\left(x_{j}\right)\right)_{6} \cap\left(\left\{y:\left\langle y-x_{j}, v\right\rangle=0\right\}\right)_{6}$. Since $E\left(X_{T}\right)<+\infty$, we can apply Lemma 3.1 (v) and get that their cardinality is controlled by some universal constant $C$.

It remains to estimate the energy outside the union of the smaller cubes. We claim that

$$
E\left(X_{T}, Q_{T}^{v}\left(y_{T}\right) \backslash \bigcup_{j=1}^{N_{S, T}} Q_{S}^{v}\left(x_{j}\right)\right) \leqq C S .
$$

To see this, note that an atom $x \in X_{T} \cap\left(Q_{T}^{v}\left(y_{T}\right) \backslash \bigcup_{j=1}^{N_{S, T}} Q_{S}^{v}\left(x_{j}\right)\right)$ can contribute to the energy only if $\left|\left\langle x-y_{T}, v\right\rangle\right| \leqq 6$. Since $E\left(X_{T}\right)<+\infty$, applying Lemma 3.1(v), we obtain

$$
\begin{aligned}
\#\left\{x \in X_{T} \cap\left(Q_{T}^{v}\left(y_{T}\right) \backslash \bigcup_{j=1}^{N_{S, T}} Q_{S}^{v}\left(x_{j}\right)\right):\left|\left\langle x-y_{T}, v\right\rangle\right| \leqq 6\right\} & \leqq C(T-S\lfloor T / S\rfloor) \\
& \leqq C S
\end{aligned}
$$

where $T-S\lfloor T / S\rfloor$ controls the length of the rightmost dark gray region in the left part of Fig. 13. In view of (2.3), this implies (7.30). Combining (7.29) and (7.30) we obtain (7.28), which concludes the proof.

As a final preparation for the proof of Theorem 2.5, we characterize the translations of lattices with touching points. To this end, we introduce the following 
notation: for given $\theta=\theta^{+}-\theta^{-} \in \mathcal{G}_{\mathbb{A}}$, we say $e^{i \theta^{+}} \tau^{+}-e^{i \theta^{-}} \tau^{-}$is a good translation and write $e^{i \theta^{+}} \tau^{+}-e^{i \theta^{-}} \tau^{-} \in \mathcal{G}_{\mathbb{T}}(\theta)$, whenever $\left(\tau^{+}, \tau^{-}\right) \in \mathbb{T}^{2}$ are such that there exist $x \in \mathscr{L}\left(\theta^{+}, \tau^{+}, 1\right)$ and $y \in \mathscr{L}\left(\theta^{-}, \tau^{-}, 1\right)$ with $|x-y|=1$. (By rotational invariance this does indeed only depend on the difference $\theta=\theta^{+}-\theta^{-}$.)

Lemma 7.6. (Properties of translations) Suppose that $\theta=\theta^{+}-\theta^{-} \in \mathcal{G}_{\mathbb{A}}$. Then $\mathcal{G}_{\mathbb{T}}(\theta)$ is contained in a finite union (of arcs) of spheres of radius 1 , namely

$$
\mathcal{G}_{\mathbb{T}}(\theta) \subset \bigcup_{x^{\prime}, y^{\prime}} \partial B_{1}\left(y^{\prime}-x^{\prime}\right),
$$

where the union is taken over the all $x^{\prime} \in e^{i \theta^{+}} \mathscr{L} \cap\left(P_{\theta^{+}, \theta^{-}}\right)_{5}$ and $y^{\prime} \in e^{i \theta^{-}} \mathscr{L} \cap$ $P_{\theta^{+}, \theta^{-}}$, where $P_{\theta^{+}, \theta^{-}}$is the fundamental parallelogram defined in (7.6). (Recall also notation (2.4).)

Proof. Consider $x \in \mathscr{L}\left(\theta^{+}, \tau^{+}, 1\right)$ and $y \in \mathscr{L}\left(\theta^{-}, \tau^{-}, 1\right)$ with $|x-y|=1$. We find a shifting vector $v \in e^{i \theta^{+}} \mathscr{L} \cap e^{i \theta^{-}} \mathscr{L}$ such that $y^{\prime}:=y-v-e^{i \theta^{-}} \tau^{-} \in$ $e^{i \theta^{-}} \mathscr{L} \cap P_{\theta^{+}, \theta^{-}}$. By defining $x^{\prime}:=x-v-e^{i \theta^{+}} \tau^{+} \in e^{i \theta^{+}} \mathscr{L}$ we clearly get

$$
1=|y-x|=\left|\left(y^{\prime}-x^{\prime}\right)-\left(e^{i \theta^{+}} \tau^{+}-e^{i \theta^{-}} \tau^{-}\right)\right| .
$$

The latter identity along with $\left|\tau^{ \pm}\right| \leqq \sqrt{3}<2$ (see (2.8)) yields $x^{\prime} \in e^{i \theta^{+}} \mathscr{L} \cap$ $\left(P_{\theta^{+}, \theta^{-}}\right)_{5}$ as well as $e^{i \theta^{+}} \tau^{+}-e^{i \theta^{-}} \tau^{-} \in \partial B_{1}\left(y^{\prime}-x^{\prime}\right)$.

We close this subsection with the proof of Theorem 2.5.

Proof of Theorem 2.5. Proof of (i),(ii). The proof of (i) follows from the definition of $\varphi$ and Lemma 6.1(i). For (ii), we use Lemma 6.1(ii) to obtain the inequality

$$
\frac{1}{2} \varphi_{\text {hex }}\left(e^{-i \theta^{+}} v\right)+\frac{1}{2} \varphi_{\text {hex }}\left(e^{-i \theta^{-}} v\right) \leqq \varphi\left(z^{+}, z^{-}, v\right) \leqq \varphi_{\text {hex }}\left(e^{-i \theta^{+}} v\right)+\varphi_{\text {hex }}\left(e^{-i \theta^{-}} v\right)
$$

for all $\left(z^{+}, z^{-}\right) \in(\mathcal{Z} \backslash\{\boldsymbol{0}\})^{2}, z^{+} \neq z^{-}$. By Lemma 6.2, $\varphi=\Phi$ (see Lemma 7.1 and Proposition 7.2), Lemma 7.1, and the definition of $\mathcal{G}_{\mathbb{T}}\left(\theta^{+}-\theta^{-}\right)$, the inequality in (7.31) can be strict only if $\theta^{+}-\theta^{-} \in \mathcal{G}_{\mathbb{A}}$ and $e^{i \theta^{+}} \tau^{+}-e^{i \theta^{-}} \tau^{-} \in \mathcal{G}_{\mathbb{T}}\left(\theta^{+}-\theta^{-}\right)$. Clearly, $\mathcal{G}_{\mathbb{A}} \subset \mathbb{A}$ is countable, see (6.2), and $\mathcal{G}_{\mathbb{T}}\left(\theta^{+}-\theta^{-}\right) \subset \mathbb{R}^{2}$ is contained in a finite union of spheres by Lemma 7.6.

Proof of (iii). Let $v_{1}, v_{2} \in \mathbb{S}^{1}, \lambda \in(0,1)$. Our goal is to prove

$$
\varphi\left(z^{+}, z^{-}, \lambda v_{1}+(1-\lambda) v_{2}\right) \leqq \lambda \varphi\left(z^{+}, z^{-}, v_{1}\right)+(1-\lambda) \varphi\left(z^{+}, z^{-}, v_{2}\right) .
$$

Assume that $\lambda v_{1}+(1-\lambda) \nu_{2} \neq 0$ (otherwise the statement is trivial) and define $v=\frac{\lambda v_{1}+(1-\lambda) v_{2}}{\left|\lambda v_{1}+(1-\lambda) v_{2}\right|} \in \mathbb{S}^{1}$. By the positive 1-homogeneity of $\varphi,(7.32)$ is equivalent to

$$
\varphi\left(z^{+}, z^{-}, v\right) \leqq \lambda_{1} \varphi\left(z^{+}, z^{-}, v_{1}\right)+\lambda_{2} \varphi\left(z^{+}, z^{-}, v_{2}\right),
$$

where $\lambda_{1}=\frac{\lambda}{\left|\lambda v_{1}+(1-\lambda) v_{2}\right|}, \lambda_{2}=\frac{1-\lambda}{\left|\lambda v_{1}+(1-\lambda) v_{2}\right|}>0$. In the following, we will prove (7.33). 
Step 1: Convexity via construction. We construct competitors for the problem $\varphi\left(z^{+}, z^{-}, v\right)$, and refer to Fig. 13 for an illustration. Fix $n \in \mathbb{N}$ such that $\lambda_{1}, \lambda_{2} \leqq$ $n / 2$. Let $1 \ll S \ll T$. As before, we assume that $S \in\left\{T_{j}\right\}_{j}$, where $\left\{T_{j}\right\}_{j}$ is the sequence identified in Proposition 7.5. For simplicity, if $S=T_{j}$, we will write $\eta_{S}$ instead of $\eta_{T_{j}}$ for the null sequence given by Proposition 7.5.

Define $N_{j}(S, T):=\left\lfloor\lambda_{j}(T-(10 n+5) S) /(n S)\right\rfloor$ for $j \in\{1,2\}$. In the following, the indices $i, j$, and $k$ are always chosen from $j \in\{1,2\}, i \in\left\{0, \ldots, N_{j}(S, T)\right\}$, and $k \in\{0, \ldots, n-1\}$ without further notice. As usual, the orthonormal vectors to $v, v_{1}, v_{2}$ obtained by clockwise rotation about $\pi / 2$ are denoted by $v^{\perp}, v_{1}^{\perp}, v_{2}^{\perp}$, respectively. From $v=\lambda_{1} \nu_{1}+\lambda_{2} \nu_{2}$ and the definition of $N_{j}(S, T)$ we get

$$
N_{1}(S, T) v_{1}^{\perp}+N_{2}(S, T) v_{2}^{\perp}=M v^{\perp}-w,
$$

where $M=(T-(10 n+5) S) /(n S)$ and $w=\alpha_{1} v_{1}^{\perp}+\alpha_{2} v_{2}^{\perp}$ for suitable $0 \leqq$ $\alpha_{1}, \alpha_{2}<1$, in particular, $|w| \leqq 2$. We set

$$
\begin{aligned}
& x_{i}^{1, k}=(-T / 2+5 S+S(M+10) k) v^{\perp}+i S v_{1}^{\perp}, \\
& x_{i}^{2, k}=x_{N_{1}(S, T)}^{1, k}+5 S v^{\perp}+i S v_{2}^{\perp},
\end{aligned}
$$

and let $X_{i}^{j, k} \subset \mathbb{R}^{2}$ be defined as a minimizer of the problem

$$
\min \left\{E_{1}\left(X, Q_{S}^{v_{j}}\left(x_{i}^{j, k}\right)\right): X=\mathscr{L}\left(z^{ \pm}\right) \text {on } \partial_{1}^{ \pm} Q_{S}^{v_{j}}\left(x_{i}^{j, k}\right)\right\} .
$$

We recall notation (2.4)-(2.5) and define

$$
\begin{aligned}
U= & \left(\left[-\frac{T}{2} \nu^{\perp} ; x_{0}^{1,0}\right]\right)_{\kappa} \cup \bigcup_{k=0}^{n-1}\left(\left[x_{N_{1}(S, T)}^{1, k} ; x_{0}^{2, k}\right]\right)_{\kappa} \cup \bigcup_{k=0}^{n-2}\left(\left[x_{N_{2}(S, T)}^{2, k} ; x_{0}^{1, k+1}\right]\right)_{\kappa} \\
& \cup\left(\left[x_{N_{2}(S, T)}^{2, n-1} ; \frac{T}{2} v^{\perp}\right]\right)_{\kappa},
\end{aligned}
$$

where $\kappa>1$ is chosen later. Note that $U$ consists of $2 n+1$ tubular neighborhoods of segments whose maximal length is bounded by $C S$. (Apart from the segment $\left[x_{N_{2}(S, T)}^{2, n-1} ; \frac{T}{2} \nu^{\perp}\right]$, this follows directly from the choice of the points $x_{i}^{j, k}$ and (7.34). For $\left[x_{N_{2}(S, T)}^{2, n-1} ; \frac{T}{2} v^{\perp}\right]$, it follows from $x_{N_{2}(S, T)}^{2, n-1}=(-T / 2+S(M+$ 10)n) $v^{\perp}-S w=(T / 2-5 S) v^{\perp}-S w$, where $|w| \leqq 2$.) We also observe that $Q_{T}^{v} \backslash\left(\bigcup_{i, j, k} Q_{S}^{v_{j}}\left(x_{i}^{j, k}\right) \cup U\right)$ consists of two connected components. The connected component intersecting $\partial_{1}^{+} Q_{T}^{v}$ is denoted by $A^{+}$and the other one is denoted by $A^{-}$. Note that the cubes $Q_{S}^{\nu_{j}}\left(x_{i}^{j, k}\right)$ do not intersect $\partial_{1}^{ \pm} Q_{T}^{v}$. We introduce the sets $A_{i}^{j, k}=Q_{10}^{v_{j}}\left(x_{i}^{j, k}+(S / 2) \nu_{j}^{\perp}\right) \cup Q_{10}^{v_{j}}\left(x_{i}^{j, k}-(S / 2) \nu_{j}^{\perp}\right)$ and let $X_{T}$ be defined by

$$
X_{T}= \begin{cases}X_{i}^{j, k} & \text { in } Q_{S}^{v_{j}}\left(x_{i}^{j, k}\right) \backslash A_{i}^{j, k}, \\ \emptyset & \text { in }\left(U \backslash\left(\bigcup_{i, j, k} Q_{S}^{v_{j}}\left(x_{i}^{j, k}\right) \cup \partial_{1}^{-} Q_{T}^{v} \cup \partial_{1}^{+} Q_{T}^{v}\right)\right) \cup \cup_{i, j, k} A_{i}^{j, k}, \\ \mathscr{L}\left(z^{ \pm}\right) & \text {in } A^{ \pm} \cup \partial_{1}^{ \pm} Q_{T}^{v} .\end{cases}
$$


For an illustration of the sets and the configuration $X_{T}$ we refer to Fig. 13. Clearly, we have $X_{T}=\mathscr{L}\left(z^{ \pm}\right)$on $\partial_{1}^{ \pm} Q_{T}^{v}$.

Step 2: Energy estimate on $X_{T}$. We now estimate the energy of $X_{T}$. First, due to the boundary conditions $X_{i}^{j, k}=\mathscr{L}\left(z^{ \pm}\right)$on $\partial_{1}^{ \pm} Q_{S}^{v_{j}}\left(x_{i}^{j, k}\right)$, one can check that for $\kappa$ big enough there holds $|x-y| \geqq 1$ for all $x, y \in X_{T}, x \neq y$ and therefore $E_{1}\left(X_{T}\right)<+\infty$. We now prove the following two sub-estimates:

$$
E_{1}\left(X_{T},\left(A^{+} \cup A^{-} \cup \partial_{1}^{+} Q_{T}^{v} \cup \partial_{1}^{-} Q_{T}^{v}\right) \cap Q_{T}^{v}\right) \leqq C n S
$$

and

$$
\begin{aligned}
& E_{1}\left(X_{T}, \bigcup_{i, j, k} Q_{S}^{v}\left(x_{i}^{j, k}\right) \cup\left(U \backslash\left(\partial_{1}^{-} Q_{T}^{v} \cup \partial_{1}^{+} Q_{T}^{v}\right)\right)\right) \\
& \quad \leqq \sum_{j=1}^{2} \frac{\lambda_{j} T}{S}\left(S\left(\varphi\left(z^{+}, z^{-}, v_{j}\right)+\eta_{S}\right)+C\right)
\end{aligned}
$$

where $\left\{\eta_{S}\right\}_{S}$ denotes a sequence with $\eta_{S} \rightarrow 0$ as $S \rightarrow+\infty$.

Proof of (7.37). For $x \in X_{T} \cap\left(A^{+} \cup A^{-} \cup \partial_{1}^{+} Q_{T}^{v} \cup \partial_{1}^{-} Q_{T}^{v}\right) \cap Q_{T}^{v}$ such that $\operatorname{dist}(x, U)>1$, there holds $\# \mathcal{N}(x)=6$. This follows from the boundary conditions of $X_{i}^{j, k}$ on every cube $Q_{S}^{v_{j}}\left(x_{i}^{j, k}\right)$ and the fact that $X_{T}=\mathscr{L}\left(z^{ \pm}\right)$in $A^{ \pm} \cup \partial_{1}^{ \pm} Q_{T}^{v}$. Therefore, in order to obtain (7.37), it suffices to estimate the cardinality of the atoms $x \in X_{T}$ lying in $(U)_{1}$. As $U$ consists of $2 n+1$ tubular neighborhoods of segments whose length is bounded by $C S$, we get $\mathcal{L}^{2}\left((U)_{2}\right) \leqq C n S$. Therefore, employing Lemma 3.1(v), we obtain \# $\left(X_{T} \cap(U)_{1}\right) \leqq C n S$. By (2.3) this implies (7.37).

Proof of (7.38):. In view of (7.36), in order to obtain (7.38), it suffices to estimate the energy contribution of atoms in $\bigcup_{i, j, k}\left(Q_{S}^{v_{j}}\left(x_{i}^{j, k}\right) \backslash A_{i}^{j, k}\right)$. For each $i, j, k$, it holds that $X_{T}=\mathscr{L}\left(z^{ \pm}\right)$on

$$
\left(\partial Q_{S}^{v_{j}}\left(x_{i}^{j, k}\right)\right)_{5} \backslash\left\{x: \pm\left\langle x-x_{i}^{j, k}, v_{j}\right\rangle \leqq C \kappa\right\},
$$

with a constant $C>0$ only depending on $v_{1}, v_{2}$ and $v$. This shows that the cardinality of $X_{T} \cap Q_{S}^{v_{j}}\left(x_{i}^{j, k}\right) \cap\left(\left(A_{i}^{j, k}\right)_{1} \cup(U)_{1}\right)$, which contains all atoms $x \in$ $X_{T} \cap Q_{S}^{v_{j}}\left(x_{i}^{j, k}\right)$ for which possibly $\#\left(\mathcal{N}(x) \cap X_{T}\right)<\#\left(\mathcal{N}(x) \cap X_{i}^{j, k}\right)$, is uniformly controlled due to Lemma $3.1(\mathrm{v})$. We thus obtain $E\left(X_{T}, Q_{S}^{v_{j}}\left(x_{i}^{j, k}\right)\right) \leqq$ $E\left(X_{i}^{j, k}, Q_{S}^{v_{j}}\left(x_{i}^{j, k}\right)\right)+C$ by (2.3). Thus, using (7.35), Propositions 7.2 and 7.5 we get

$$
E\left(X_{T}, Q_{S}^{v_{j}}\left(x_{i}^{j, k}\right)\right) \leqq E\left(X_{i}^{j, k}, Q_{S}^{v_{j}}\left(x_{i}^{j, k}\right)\right)+C \leqq S\left(\varphi\left(z^{+}, z^{-}, v_{j}\right)+\eta_{S}\right)+C .
$$

For $j \in\{1,2\}$, we find that

$$
\#\left\{(i, k): i=0, \ldots, N_{j}(S, T), k=0, \ldots, n-1\right\}
$$




$$
=n\left(\left\lfloor\frac{\lambda_{j}(T-(10 n+5) S)}{n S}\right\rfloor+1\right) \leqq \frac{\lambda_{j} T}{S} .
$$

This, along with (7.39), yields (7.38).

Step 3: Conclusion. Noting that

$$
\min \left\{E_{1}\left(X, Q_{T}^{v}\right): X=\mathscr{L}\left(z^{ \pm}\right) \text {on } \partial_{1}^{ \pm} Q_{T}^{v}\right\} \leqq E_{1}\left(X_{T}, Q_{T}^{v}\right),
$$

and using (7.37)-(7.38) as well as Lemma 3.1(iv), we have

$$
\begin{aligned}
\min & \left\{E_{1}\left(X, Q_{T}^{v}\right): X=\mathscr{L}\left(z^{ \pm}\right) \text {on } \partial_{1}^{ \pm} Q_{T}^{v}\right\} \leqq \lambda_{1} T\left(\varphi\left(z^{+}, z^{-}, v_{1}\right)+\eta_{S}\right)+C \lambda_{1} T / S \\
& +\lambda_{2} T\left(\varphi\left(z^{+}, z^{-}, v_{2}\right)+\eta_{S}\right)+C \lambda_{2} T / S+C n S .
\end{aligned}
$$

Dividing by $T$, letting first $T \rightarrow+\infty$, and then $S \rightarrow+\infty$, we obtain (7.33) by Proposition 7.2, where we also use $\eta_{S} \rightarrow 0$. This concludes the proof of (iii).

Proof of (iv). Let $z^{ \pm}=\left(\theta^{ \pm}, \tau^{ \pm}, 1\right), v \in \mathbb{S}^{1}$, and $\theta \in \mathbb{A}$. Our goal is to prove

$$
\begin{aligned}
& \varphi\left(\left(\theta^{+}+\theta, \tau^{+}, 1\right),\left(\theta^{-}+\theta, \tau^{-}, 1\right), e^{i \theta} v\right) \\
& \quad=\varphi\left(\left(\theta^{+}, \tau^{+}, 1\right),\left(\theta^{-}, \tau^{-}, 1\right), \nu\right) .
\end{aligned}
$$

Due to Proposition 7.2, for every $T>0$ we can choose $X_{T} \subset \mathbb{R}^{2}$, such that $X_{T}=\mathscr{L}\left(\left(\theta^{ \pm}, \tau^{ \pm}, 1\right)\right)$ on $\partial_{1}^{ \pm} Q_{T}^{v}$ and such that

$$
\lim _{T \rightarrow+\infty} \frac{1}{T} E_{1}\left(X_{T}, Q_{T}^{v}\right)=\varphi\left(\left(\theta^{+}, \tau^{+}, 1\right),\left(\theta^{-}, \tau^{-}, 1\right), v\right) .
$$

We set $X_{T}^{\theta}=e^{i \theta} X_{T}$. Then $X_{T}^{\theta}=\mathscr{L}\left(\left(\theta^{ \pm}+\theta, \tau^{ \pm}, 1\right)\right)$ on $\partial_{1}^{ \pm} Q_{T}^{v_{\theta}}$, where $v_{\theta}=e^{i \theta} v$. Applying Proposition 7.2, Lemma 3.1(i), and (7.41), we obtain

$$
\begin{gathered}
\varphi\left(\left(\theta^{+}+\theta, \tau^{+}, 1\right),\left(\theta^{-}+\theta, \tau^{-}, 1\right), e^{i \theta} \nu\right) \leqq \liminf _{T \rightarrow+\infty} \frac{1}{T} E_{1}\left(X_{T}^{\theta}, Q_{T}^{\nu_{\theta}}\right) \\
=\lim _{T \rightarrow+\infty} \frac{1}{T} E_{1}\left(X_{T}, Q_{T}^{v}\right)=\varphi\left(\left(\theta^{+}, \tau^{+}, 1\right),\left(\theta^{-}, \tau^{-}, 1\right), v\right) .
\end{gathered}
$$

This implies one inequality in (7.40). The other inequality follows by repeating the argument for $\left(\tilde{\theta}^{ \pm}, \tau^{ \pm}, 1\right)=\left(\theta^{ \pm}+\theta, \tau^{ \pm}, 1\right), \tilde{v}=e^{i \theta} v$, and $\tilde{\theta}=-\theta$. This concludes the proof of (iv).

Proof of $(v)$. Let $z^{ \pm}=\left(\theta^{ \pm}, \tau^{ \pm}, 1\right), v \in \mathbb{S}^{1}$, and $\tau \in \mathbb{T}$. Our goal is to prove

$$
\begin{aligned}
& \varphi\left(\left(\theta^{+}, \tau^{+}+e^{-i \theta^{+}} \tau, 1\right),\left(\theta^{-}, \tau^{-}+e^{-i \theta^{-}} \tau, 1\right), \nu\right) \\
& \quad=\varphi\left(\left(\theta^{+}, \tau^{+}, 1\right),\left(\theta^{-} \tau^{-}, 1\right), \nu\right) .
\end{aligned}
$$

Due to Proposition 7.2, for every $T>0$ we can choose $X_{T} \subset \mathbb{R}^{2}$, such that $X_{T}=\mathscr{L}\left(\left(\theta^{ \pm}, \tau^{ \pm}, 1\right)\right)$ on $\partial_{1}^{ \pm} Q_{T}^{v}$ and such that (7.41) holds. We set $X_{T}^{\tau}=X_{T}+\tau$. Then $X_{T}^{\tau}=\mathscr{L}\left(\left(\theta^{ \pm}, \tau^{ \pm}+e^{-i \theta^{ \pm}} \tau, 1\right)\right)$ on $\partial_{1}^{ \pm} Q_{T}^{v}(\tau)$. Applying Proposition 7.2, Lemma 3.1(i), and (7.41), we get

$$
\varphi\left(\left(\theta^{+}, \tau^{+}+e^{-i \theta^{+}} \tau, 1\right),\left(\theta^{-}, \tau^{-}+e^{-i \theta^{-}} \tau, 1\right), \nu\right)
$$




$$
\begin{aligned}
& \leqq \liminf _{T \rightarrow+\infty} \frac{1}{T} E_{1}\left(X_{T}^{\tau}, Q_{T}^{\nu}(\tau)\right)=\lim _{T \rightarrow+\infty} \frac{1}{T} E_{1}\left(X_{T}, Q_{T}^{\nu}\right) \\
& =\varphi\left(\left(\theta^{+}, \tau^{+}, 1\right),\left(\theta^{-}, \tau^{-}, 1\right), \nu\right) .
\end{aligned}
$$

This yields one inequality of (7.42). The other inequality follows by repeating the argument for $\left(\theta^{ \pm}, \tilde{\tau}^{ \pm}, 1\right)=\left(\theta^{ \pm}, \tau^{ \pm}+e^{-i \theta^{ \pm}} \tau, 1\right)$ and $\tilde{\tau}=-\tau$. This concludes the proof of $(\mathrm{v})$.

Acknowledgements. MF and LK acknowledge support by the DFG Projects FR 4083/11, FR 4083/3-1 and by the Deutsche Forschungsgemeinschaft (DFG, German Research Foundation) under Germany's Excellence Strategy EXC 2044-390685587, Mathematics Münster: Dynamics-Geometry-Structure.

Funding Open Access funding enabled and organized by Projekt DEAL.

Open Access This article is licensed under a Creative Commons Attribution 4.0 International License, which permits use, sharing, adaptation, distribution and reproduction in any medium or format, as long as you give appropriate credit to the original author(s) and the source, provide a link to the Creative Commons licence, and indicate if changes were made. The images or other third party material in this article are included in the article's Creative Commons licence, unless indicated otherwise in a credit line to the material. If material is not included in the article's Creative Commons licence and your intended use is not permitted by statutory regulation or exceeds the permitted use, you will need to obtain permission directly from the copyright holder. To view a copy of this licence, visit http://creativecommons.org/ licenses/by/4.0/.

Publisher's Note Springer Nature remains neutral with regard to jurisdictional claims in published maps and institutional affiliations.

\section{References}

1. Allinger, N.L.: Molecular Structure: Understanding Steric and Electronic Effects from Molecular Mechanics. Wiley, New York 2010

2. Ambrosio, L., Fusco, N., Pallara, D.: Functions of Bounded Variation and Free Discontinuity Problems. Oxford University Press, Oxford 2000

3. Au Yeung, Y., Friesecke, G., Schmidt, B.: Minimizing atomic configurations of short range pair potentials in two dimensions: crystallization in the Wulff-shape. Calc. Var. Partial Differ. Eq. 44, 81-100, 2012

4. Bach, A., Braides, A., Cicalese, M.: Discrete-to-continuum limits of multi-body systems with bulk and surface long-range interactions. SIAM J. Math. Anal. (to appear). Preprint at arXiv:1910.00346

5. Barroso, A.C., Fonseca, I.: Anisotropic singular perturbations-the vectorial case. Proc. R. Soc. Edinb. Sect. A 124, 527-571, 1994

6. BÉTERmin, L., KNÜPfER, H., Nolte, F.: Note on crystallization for alternating particle chains. Preprint at arXiv: 1804.05743

7. Blanc, X., Lewin, M.: The crystallization conjecture: a review. EMS Surv. Math. Sci. 2, 225-306, 2015

8. Braides, A.: $\Gamma$-convergence for Beginners. Oxford University Press, Oxford 2002

9. Braides, A., Conti, S., Garroni, A.: Density of polyhedral partitions. Calc. Var. Partial Differ. Equ. 56, Paper No. 28, 2017

10. Cagnetti, F., Dal Maso, G., Scardia, L., Zeppieri, C.I.: $\Gamma$-convergence of freediscontinuity problems. Ann. Inst. H. Poincaré Anal. Non Linéaire 36, 1035-1079, 2019 
11. Cicalese, M., Leonardi, G.P.: Maximal fluctuations on periodic lattices: an approach via quantitative Wulff inequalities. Commun. Math. Phys. 375, 1931-1944, 2020

12. Conti, S., FonseCA, I., LeOni, G.: A $\Gamma$-convergence result for the two-gradient theory of phase transitions. Commun. Pure Appl. Math. 55, 857-936, 2002

13. Conti, S., Schweizer, B.: Rigidity and gamma convergence for solid-solid phase transitions with $S O(2)$ invariance. Commun. Pure Appl. Math. 59, 830-868, 2006

14. Dal Maso, G.: An Introduction to $\Gamma$-convergence. Birkhäuser, Boston 1993

15. Davoli, E., Friedrich, M.: Two-well rigidity and multidimensional sharp-interface limits for solid-solid phase transitions. Calc. Var. Partial Differ. Equ. 59, Paper No. 44, 2020

16. Davoli, E., Piovano, P., Stefanelli, U.: Wulff shape emergence in graphene. Math. Models Methods Appl. Sci. 26, 2277-2310, 2016

17. Davoli, E., Piovano, P., Stefanelli, U.: Sharp $N^{3 / 4}$ law for the minimizers of the edge-isoperimetric problem on the triangular lattice. J. Nonlin. Sci. 27, 627-660, 2017

18. De Luca, L., Friesecke, G.: Crystallization in two dimensions and a discrete GaussBonnet Theorem. J. Nonlinear Sci. 28, 69-90, 2017

19. De Luca, L., Friesecke, G.: Classification of particle numbers with unique HeitmannRadin minimizer. J. Stat. Phys. 167, 1586-1592, 2017

20. De Luca, L., Novaga, M., Ponsiglione, M.: Gamma-convergence of the HeitmannRadin sticky disc energy to the crystalline perimeter. J. Nonlinear Sci. 29, 1273-1299, 2019

21. E, W., LI, D.: On the crystallization of 2D hexagonal lattices. Commun. Math. Phys. 286, 1099-1140, 2009

22. Evans, L.C., GariePY, R.F.: Measure Theory and Fine Properties of Functions. CRC Press, Boca Raton 1992

23. Fanzon, S., Palombaro, M., Ponsiglione, M.: Derivation of linearized polycrystals from a two-dimensional system of edge dislocations. SIAM J. Math. Anal. 51, 39563981, 2019

24. Flatley, L.C., Theil, F.: Face-centered cubic crystallization of atomistic configurations. Arch. Ration. Mech. Anal. 218, 363-416, 2015

25. Fonseca, I., Tartar, L.: The gradient theory of phase transitions for systems with two potential wells. Proc. R. Soc. Edinb. Sect. A 111, 89-102, 1989

26. Friedrich, M., Kreutz, L.: Crystallization in the hexagonal lattice for ionic dimers. Math. Models Methods Appl. Sci. 29, 1853-1900, 2019

27. Friedrich, M., Kreutz, L.: Finite crystallization and Wulff shape emergence for ionic compounds in the square lattice. Nonlinearity 33, 1240-1296, 2020

28. Friedrich, M., Solombrino, F.: Functionals defined on piecewise rigid functions: Integral representation and $\Gamma$-convergence. Arch. Ration. Mech. Anal. 236, 1325-1387, 2020

29. Friedrich, M., Stefanelli, U.: Crystallization in a one-dimensional periodic landscape. J. Stat. Phys. 179, 485-501, 2020

30. Friesecke, G., Theil, F.: Molecular geometry optimization, models. In: EngQuist, B. (ed.) The Encyclopedia of Applied and Computational Mathematics. Springer, Berlin 2015

31. Gardner, C.S., Radin, C.: The infinite-volume ground state of the Lennard-Jones potential. J. Stat. Phys. 20, 719-724, 1979

32. Harborth, H.: Lösung zu Problem 664 a. Elem. Math. 29, 14-15, 1974

33. Heitmann, R., Radin, C.: The ground state for sticky disks. J. Stat. Phys. 22, 281-287, 1980

34. Jansen, S., König, W., Schmidt, B., Theil, F.: Surface energy and boundary layers for a chain of atoms at low temperature. Submitted. Preprint at arxiv:1904.06169, 2019

35. Jansen, S., König, W., Schmidt, B., Theil, F.: Distribution of cracks in a chain of atoms at low temperature. In preparation, 2020 
36. Kitavtsev, G., Luckhaus, S., Rüland, A.: Surface energies emerging in a microscopic, two-dimensional two-well problem. Proc. R. Soc. Edinb. Sect. A 147, 10411089,2017

37. Lewars, E.G.: Computational Chemistry, 2nd edn. Springer, New York 2011

38. Lauteri, G., Luckhaus, S.: Submitted. Preprint at arxiv:1608.06155, 2016

39. Mainini, E., Piovano, P., Schmidt, B., Stefanelli, U.: $N^{3 / 4}$ law in the cubic lattice. J. Stat. Phys. 176, 1480-1499, 2019

40. Mainini, E., Piovano, P., Stefanelli, U.: Finite crystallization in the square lattice. Nonlinearity 27, 717-737, 2014

41. Mainini, E., Schmidt, B.: Maximal fluctuations around the Wulff shape for edgeisoperimetric sets in $\mathbb{Z}^{d}$ : a sharp scaling law. Submitted. Preprint at arXiv:2003.01679, 2020

42. Mainini, E., Stefanelli, U.: Crystallization in carbon nanostructures. Commun. Math. Phys. 328, 545-571, 2014

43. Radin, C.: The ground state for soft disks. J. Stat. Phys. 26, 365-373, 1981

44. Read, W.T., Shockley, W.: Dislocation models of crystal grain boundaries. Phys. Rev. 78, 275-289, 1950

45. Schmidt, B.: Ground states of the 2D sticky disc model: fine properties and $N^{3 / 4}$ law for the deviation from the asymptotic Wulff shape. J. Stat. Phys. 153, 727-738, 2013

46. STERnberg, P.: The effect of a singular perturbation on nonconvex variational problems. Arch. Ration. Mech. Anal. 101, 209-260, 1988

47. Theil, F.: A proof of crystallization in two dimensions. Commun. Math. Phys. 262, 209-236, 2006

\author{
M. FRIEDRICH, L. KREUTZ \\ Applied Mathematics Münster, \\ University of Münster, \\ Einsteinstrasse 62, \\ 48149 Münster \\ Germany. \\ e-mail: manuel.friedrich@uni-muenster.de \\ L. KReutZ \\ e-mail: lkreutz@uni-muenster.de \\ and \\ B. SCHMIDT \\ Institut für Mathematik, \\ Universität Augsburg, \\ Universitätsstr. 14, \\ 86159 Augsburg \\ Germany. \\ e-mail: bernd.schmidt@math.uni-augsburg.de
}

(Received June 3, 2020 / Accepted January 29, 2021)

Published online March 12, 2021

(c) The Author(s) (2021) 